\title{
EXTENSÕES VIRTUAIS
}

Sérgio Ferreira Cortizo

Tese de Doutorado apresentada à Faculdade de Filosofia, Letras e Ciências Humanas da Universidade de São Paulo.

Orientador: Prof. Newton C. A. Da Costa 
à Roberta 


\section{Agradecimentos}

Gostaria de expressar minha sincera gratidão a todos aqueles que contribuíram, direta ou indiretamente, para a elaboração deste trabalho.

Sou particularmente grato ao Prof. Newton da Costa, meu orientador. Estou certo que não há outra pessoa, em todo o mundo, que pudesse ter desempenhado melhor este papel.

Não poderia deixar de destacar também meu irmão, Paulo Cortizo; e meus amigos Paulo Marques, Edélcio de Souza e Augusto Lobo; que colaboraram através de conversas e discussões para o desenvolvimento das idéias aqui apresentadas. 


\section{Índice}

Introdução 11

$\begin{array}{lll}1 & \text { Extensões Virtuais } & 18\end{array}$

1.1 Objetos, Predicados e Funcionais _ . . . . . . . . . . . . . 18

1.2 Objetos, Predicados e Funcionais Virtuais . . . . . . . . . . . . . . . . . . . . . . . . . . . . . . . .

1.3 Transferência de Objetos, Predicados e Funcionais . . . . . . . . . . . . . . . . . . . . . . . . . . . .

1.4 O Teorema da Extensão Virtual . . . . . . . . . . . . . . . . . . . . 22

1.5 Prova do Teorema da Extensão Virtual . . . . . . . . . . . . . . . . . 24

1.6 Semelhança e Dessemelhança . . . . . . . . . . . . . . . . . 33

1.7 Inclusão Lógica e Embaralhamento . . . . . . . . . . . . . . . . . . . . 35

1.8 Mistura Lógica . . . . . . . . . . . . . . . . . . . . . . . 37

1.9 Decomposição Lógica . . . . . . . . . . . . . . . . . . . . . . 38

$2 \quad$ Cálculo de Funções Reais $\quad 41$

2.1 Números Virtuais . . . . . . . . . . . . . . . . . . . . . . . . 41

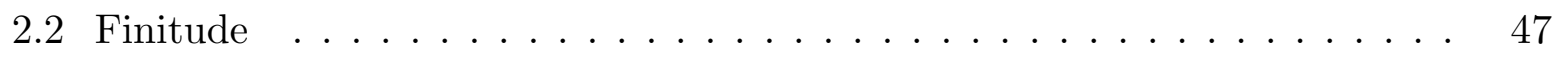

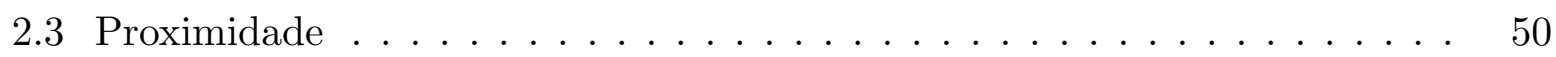

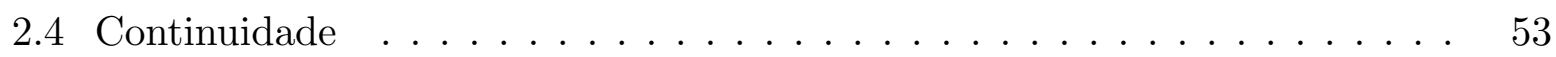

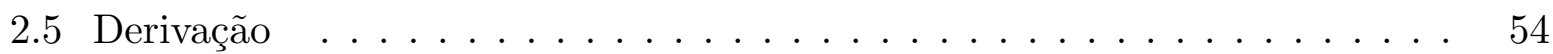

2.6 Quantidades Virtuais . . . . . . . . . . . . . . . . . 60

2.7 Integração . . . . . . . . . . . . . . . . . . . . . 66 
3.1 Conjuntos Virtuais . . . . . . . . . . . . . . . . 70

3.2 Funções Virtuais . . . . . . . . . . . . . . . . . . . . . . . 73

3.3 Virtualização de Parâmetros . . . . . . . . . . . . . . . . 75

3.4 Continuidade Virtual . . . . . . . . . . . . . . . . . . . 81

3.5 Derivação Virtual . . . . . . . . . . . . . . . . . . 83

3.6 Integração Virtual f . . . . . . . . . . . . . . . . . . . . 86

$4 \quad$ O Cálculo Delta de Dirac $\quad 92$

4.1 Funções de Dirac . . . . . . . . . . . . . . . . . . . . . . . . 92

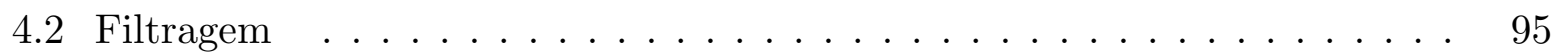

4.3 Equivalência de Dirac . . . . . . . . . . . . . . . . . . 97

4.4 A Regra de Composição . . . . . . . . . . . . . . . . . . . . . . . . . . . 99

4.5 Contração de Funções de Dirac . . . . . . . . . . . . . . . . . . . . 102

4.6 Funções de Dirac Diferenciáveis . . . . . . . . . . . . . . . . . 105

$\begin{array}{lr}\text { Epílogo } & 108\end{array}$

$\begin{array}{lr}\text { Apêndice } & 110\end{array}$

$\begin{array}{ll}\text { Bibliografia } & 113\end{array}$ 


\section{Introdução}

O Conhecimento Científico fundamenta-se em uma sólida ligação entre os métodos empíricos das ciências naturais e o pensamento matemático abstrato. Esta ligação pode ser claramente observada ao longo de toda a história da Ciência Moderna, desde seu advento, tradicionalmente associado à obra de Galileu. Todos conhecemos as célebres palavras do sábio florentino a respeito da linguagem em que está escrito "o livro da natureza".

Newton, o principal continuador do trabalho de Galileu, e talvez o maior físico de todos os tempos, foi também um dos criadores do Cálculo Infinitesimal, do qual boa parte da matemática contemporânea pode ser considerada um desdobramento. Grandes cientistas dos séculos XVIII e XIX, como Euler, Lagrange e Gauss, contribuíram diretamente para o progresso da Matemática tanto quanto para o da Física.

Na segunda metade do século XIX, entretanto, surgiu entre os matemáticos um outro ponto de vista a respeito de sua própria atividade de pesquisa, segundo o qual a investigação matemática deve ser "independente" das ciências empíricas. Este sentimento difundiu-se a ponto de se tornar, neste século, predominante entre os matemáticos profissionais.

Uma apresentação extremamente lúcida desse processo pode ser encontrada no livro Mathematics, The Loss of Certainty [6], de autoria de M. Kline, um profundo conhecedor da história do pensamento matemático [5]. No capítulo intitulado The Isolation of Mathematics, Kline expõe o conflito permanente, ao longo deste século, entre duas visões opostas sobre a natureza do conhecimento matemático.

A discussão claramente envolve muitas sutilezas, aspectos subjetivos, e deixa margem para mal-entendidos e confusões de toda sorte. Apesar disto, acreditamos ser extremamente relevante aprofundá-la, pois ela determina, em larga medida, os critérios utilizados na avaliação da qualidade da pesquisa matemática atual, condicionando assim, fortemente, 
os rumos das investigações. A fim de destacar o que consideramos como o cerne da questão, introduziremos a seguir duas palavras para identificar as posições extremas em debate.

Chamaremos de separatista a visão surgida no final do século passado, que defende a independência da pesquisa matemática em relação às ciências empíricas. O sentido dado atualmente à classificação da investigação matemática em "pura" ou "aplicada" reflete bem esta mentalidade: o valor de um trabalho de matemática pura é medido unicamente pela sua complexidade interna e seu rigor formal, à matemática aplicada cabe utilizar os resultados obtidos, na medida do possível, nas demais áreas do conhecimento humano. A unilateralidade deste ponto de vista é clara: não deve haver influência externa sobre a pesquisa matemática pura, que é "soberana" para seguir o curso de seu próprio desenvolvimento.

Por outro lado, chamaremos de unionista o ponto de vista oposto, que vê a Matemática como parte integrante do corpo do Conhecimento Científico, e considera esta relação como fundamento de toda atividade de investigação matemática. Segundo esta perspectiva, deve haver tanta influência das ciências empíricas sobre o pensamento matemático quanto houver no sentido contrário, ou seja, esta interação deve ser bilateral, com influências recíprocas. Assim, a divisão da pesquisa em "pura" e "aplicada" não deve ser utilizada como pretexto ou justificativa para o isolamento da Matemática.

Entre esses dois pólos há, é claro, diversas posições intermediárias, que atribuem à matemática diferentes graus de autonomia. A julgar pelas passagens citadas por Kline, poderíamos dizer que Hardy, Dickson, Stone e Dieudonné estão entre os matemáticos que adotam a visão separatista, enquanto entre os unionistas temos Klein, Poincaré, Courant, Birkhoff, Synge, e von Neumann.

Falar em separatistas contra unionistas sugere uma guerra de secessão no interior da Ciência. Esta metáfora não nos parece descabida: acontecimentos desta natureza fazem parte da dinâmica do desenvolvimento de toda instituição humana, com a Ciência não haveria de ser diferente, tendo em vista o seu caráter social. A seguir, um trecho* do livro de Kline ([6], p. 303).

The break from science has been accelerated during this century. Today it is common to hear and read mathematicians' declarations of independence from science. Mathematicians no longer hesitate to speak freely of their interest solely in mathematics proper and their indifference to science. Though no precise statistics are available, about ninety percent of the mathematicians active today are ignorant of science and are quite content to remain in that blissful state. Despite the history and some opposition, the

\footnotetext{
* Apresentamos uma tradução para o Português de todas as citações deste trabalho em um apêndice, pág. 110.
} 
trend to abstraction, to generalization for the sake of generalization, and to the pursuit of arbitrarily chosen problems has continued. The reasonable need for study of an entire class of problems in order to get at the essence of a problem has become an excuse for tackling generalities and abstractions in and for themselves.

Over the centuries man created such grand structures as Euclidean geometry, the Ptolemaic theory, the heliocentric theory, Newtonian mechanics, electromagnetic theory, and in recent times relativity and quantum theory. In all of these and in other significant and powerful bodies of science, mathematics as we know is the method of construction, the framework, and indeed the essence. Mathematical theories have enabled us to know something of nature, to embrace in comprehensive intelligible accounts varieties of seemingly diverse phenomena. Mathematical theories have revealed whatever order and plan man has found in nature and have given us mastery or partial mastery over vast domains.

But most mathematicians have abandoned their traditions and heritage. The pregnant messages that nature sends to the senses now fall on closed eyes and inattentive ears. Mathematicians are living on the reputation earned by their predecessors and still expect the acclaim and support that the older work warranted. The pure mathematicians have gone further. They have expelled the applied mathematicians from their fraternity in the hope that by cornering the honorable title of mathematician they alone will gain the glory accorded to their predecessors. They have thrown away their rich source of ideas and are now spending their previously accumulated wealth. They have followed a gleam that has led them out of this world. It is true that some, aware of the noble tradition that motivated mathematical research in the past and warranted the honor accorded to the Newtons and Gausses, still claim potential value of their mathematical work for science. They speak of creating models for science. But in truth they are not concerned with this goal. In fact, since most modern mathematicians know no science, they can't be creating models. They prefer to remains virgins rather than to bed with science. On the whole mathematics is now turned inward; it feeds on itself; and it is extremely unlikely, if one may judge by what happened in the past, that most of modern mathematical research will ever contribute to the advancement of science; mathematics may be doomed to grope in darkness. Mathematics is now an almost entirely self-contained enterprise. Moving in directions determined by its own criteria of relevance and excellence, it is even proud of its independence from outside problems, motivations, and inspirations. It no longer has unity and purpose.

As idéias que apresentaremos neste trabalho refletem uma visão da matemática profundamente unionista. Admitimos como evidente que as diversas áreas do saber científico têm suas particularidades: desempenham papéis diferentes no conjunto geral, estudam temas bastante distintos e usam métodos de investigação próprios. É claro que estas particularidades conferem uma relativa autonomia a cada uma delas. Mas acreditamos que as diversas áreas da Ciência estejam interligadas como as partes de um organismo vivo, e que apenas este inter-relacionamento orgânico garante a relevância e a durabilidade histórica dos resultados obtidos em cada uma delas. 
Ao longo deste século, o incremento da "divisão do trabalho científico" levou os investigadores a um nível de especialização técnica sem precedentes. Chegamos a um ponto em que toda pesquisa inter-disciplinar é vista com suspeita, e o adjetivo "generalista" tornouse pejorativo. Este processo histórico colaborou bastante para o aumento do sentimento separatista entre os matemáticos. O preconceito contra a pesquisa inter-disciplinar, entretanto, é fruto de uma confusão. O objetivo da investigação científica é aprimorar nosso conhecimento racional sobre o mundo, a especialização é apenas um meio para aumentar a eficiência deste trabalho. Acreditar que apenas a investigação altamente especializada pode colaborar para o progresso do conhecimento é confundir os meios com os fins. Todos reconhecemos que, em certa medida, a especialização técnica é útil, mas devemos usála criteriosamente, pois são as relações inter-disciplinares que dão sentido à divisão do trabalho.

A visão separatista considera o conhecimento matemático essencialmente diferente do conhecimento das ciências empíricas: este estaria baseado em inferências e induções, enquanto aquele seria puramente dedutivo, e portanto poderia alcançar, através do rigor formal, uma certeza matemática independente de confirmação empírica. A crença no caráter exclusivamente dedutivo da Matemática pressupõe que ela seja absolutamente livre para escolher seus temas e métodos de estudo, que suas definições e axiomas possam ser escolhidos arbitrariamente. Esta liberdade, entretanto, é apenas uma hipótese de trabalho, e não uma afirmação que possa ser comprovada ou refutada.

Pela sua própria natureza não-empírica, pela sua ênfase no raciocínio dedutivo, pelo seu apego ao rigor formal, a investigação matemática atual tende fortemente a desgarrarse das outras ciências: é muito mais fácil relacionar as idéias matemáticas umas com as outras do que com aquelas não-matemáticas. Apesar disto, entendemos que a vitalidade histórica da Matemática não pode prescindir do seu vínculo com o corpo do Conhecimento Científico. Ao nosso ver, este vínculo é o verdadeiro fundamento da Matemática.

Consideramos como a principal lição ensinada pela História da Ciência: o conhecimento humano progride como um todo, e os verdadeiros avanços em cada área são aqueles que se integram neste progresso global. Acreditamos que, futuramente, a época separatista que estamos vivendo será vista como uma espécie de "deslumbramento coletivo" que contaminou a quase totalidade dos matemáticos profissionais por mais de cem anos. Consequentemente, muito pouco da Matemática "pura" desenvolvida hoje em dia será perpetuado no trabalho das gerações seguintes.

Discutiremos a seguir um exemplo notável do fato apontado por Kline na citação acima: "The pregnant messages that nature sends to the senses now fall on closed eyes and inattentive ears". 
Na primeira metade deste século, o físico teórico P. Dirac introduziu no formalismo matemático da Mecânica Quântica ([2], p. 58) o que podemos chamar de "Cálculo Delta de Dirac". Trata-se de um conjunto de regras operacionais envolvendo sua célebre "função delta":

$$
\begin{gathered}
\delta(x)= \begin{cases}\infty & , \text { se } x=0 \\
0 & , \text { se } x \neq 0 .\end{cases} \\
\int_{-\infty}^{\infty} \delta(x) d x=1 .
\end{gathered}
$$

Desde que foi criado, este Cálculo tem-se mostrado uma técnica extremamente útil e conveniente, e tem sido amplamente utilizado, não apenas no contexto original da Mecânica Quântica, mas em praticamente toda a literatura da Física. Entre suas regras operacionais temos a propriedade de filtragem da função delta: se $f$ é uma função real contínua em $a \in \mathbf{R}$ então:

$$
\int_{-\infty}^{\infty} \delta(x-a) f(x) d x=f(a)
$$

e a fórmula:

$$
\delta\left(x^{2}-a^{2}\right)=\frac{1}{2 a}[\delta(x-a)+\delta(x+a)] \quad(a>0) .
$$

Segue um exemplo típico da forma como estas equações são utilizadas pelos físicos nos seus cálculos com a função delta:

$$
\begin{aligned}
\int_{-\infty}^{\infty} \delta\left(x^{2}-9\right) e^{x} d x & =\int_{-\infty}^{\infty} \frac{1}{6}[\delta(x-3)+\delta(x+3)] e^{x} d x \\
& =\int_{-\infty}^{\infty} \frac{1}{6} \delta(x-3) e^{x} d x+\int_{-\infty}^{\infty} \frac{1}{6} \delta(x+3) e^{x} d x \\
& =\frac{1}{6} e^{3}+\frac{1}{6} e^{-3}=\frac{1}{6}\left(e^{3}+\frac{1}{e^{3}}\right)
\end{aligned}
$$

O surgimento do Cálculo Delta precipitou a formalização de uma série de idéias matemáticas, que convergiram rapidamente, em meados deste século, para o conceito formal de distribuição, que pode ser definido de várias formas equivalentes (pequenas diferenças técnicas não serão relevantes na nossa discussão). Uma exposição bastante detalhada da história deste conceito pode ser encontrada no ótimo livro [10] de J. Lützen, que fornece também uma extensa bibliografia sobre o tema.

Muitos pesquisadores acreditam que o Cálculo Delta de Dirac tenha sido formalizado matematicamente pela idéia de distribuição. Entretanto, é fácil ver que considerando a função delta como uma distribuição, não conseguimos formalizar nem mesmo as regras operacionais mais elementares e essenciais do Cálculo Delta. 
O exemplo típico de cálculo com a função delta apresentado acima mostra isto claramente: considerando a função delta como uma distribuição, não podemos justificar a fórmula básica:

$$
\delta\left(x^{2}-a^{2}\right)=\frac{1}{2 a}[\delta(x-a)+\delta(x+a)] \quad(a>0) .
$$

Aliás, considerando a função delta como uma distribuição, o membro esquerdo desta igualdade não pode sequer ser escrito, pois só é possível definir a composição de uma distribuição com uma função real cuja derivada não se anule.

É importante observar que a fórmula acima não é apenas uma regra operacional de importância secundária. Ela é um caso particular de uma regra de composição mais geral, bem conhecida e de uso cotidiano na Física Teórica:

$$
\delta[g(x)]=\sum_{i=1}^{n} \frac{1}{\left|g^{\prime}\left(a_{i}\right)\right|} \delta\left(x-a_{i}\right),
$$

onde $a_{1}, a_{2}, \ldots, a_{n}$ são as raízes simples de uma função real $g$. É consenso entre os físicos que desta regra provém toda a utilidade e a conveniência do Cálculo Delta, e que, portanto, ela constitui sua própria essência.

Muitos matemáticos já atentaram para as graves limitações operacionais do conceito de distribuição. Por exemplo, em Courant-Hilbert ([1], p. 788) lemos:

Introducing ideal functions may appear a sweeping extension of ordinary calculus. Yet, in the realm of ideal functions not all operations of classical calculus can be carried out. Thus the advantage of securing unrestricted differentiability is partly offset by the loss of freedom in multiplying functions or in forming composite functions. It is not even completely true that an ideal function of several variables becomes an ideal function of fewer variables if some of the others are kept constant in a domain of definition.

Aparentemente, no entanto, ainda passa desapercebido que nem mesmo as idéias matemáticas subjacentes ao Cálculo Delta podem ser formalizadas pelo conceito de distribuição.

Essas idéias matemáticas são, contudo, extremamente simples, como podemos verificar por um estudo mais detalhado do exemplo acima de cálculo. Podemos traduzir a concepção de Dirac sobre sua função delta para a linguagem da matemática contemporânea definindo uma sequência delta como uma sequência de funções reais $f_{n}: \mathbf{R} \rightarrow \mathbf{R}_{+}$tais que:

(i) existe uma sequência de números reais positivos $\left(x_{n}\right) \operatorname{com} x_{n} \rightarrow 0$ e

$$
|x|>x_{n} \text { implica } f_{n}(x)=0, \mathrm{e}
$$


(ii) as funções $f_{n}$ são todas integráveis com

$$
\int_{-\infty}^{\infty} f_{n}(x) d x=1
$$

Nestes termos, o exemplo de cálculo com a função delta acima equivale à seguinte afirmação: tomando uma sequência delta $f_{n}$ qualquer, teremos necessariamente:

$$
\begin{aligned}
\lim _{n \rightarrow \infty} \int_{-\infty}^{\infty} f_{n}\left(x^{2}-9\right) e^{x} d x & =\lim _{n \rightarrow \infty} \int_{-\infty}^{\infty} \frac{1}{6}\left[f_{n}(x-3)+f_{n}(x+3)\right] e^{x} d x \\
& =\lim _{n \rightarrow \infty} \int_{-\infty}^{\infty} \frac{1}{6} f_{n}(x-3) e^{x} d x+\lim _{n \rightarrow \infty} \int_{-\infty}^{\infty} \frac{1}{6} f_{n}(x+3) e^{x} d x \\
& =\frac{1}{6} e^{3}+\frac{1}{6} e^{-3}=\frac{1}{6}\left(e^{3}+\frac{1}{e^{3}}\right) .
\end{aligned}
$$

Vemos então que a propriedade de filtragem da função delta expressa simplesmente uma passagem ao limite de justificativa matematicamente elementar:

$$
\lim _{n \rightarrow \infty} \int_{-\infty}^{\infty} f_{n}(x-a) h(x) d x=h(a)
$$

para qualquer sequência delta $f_{n}$ e qualquer função real integrável $h$ que seja contínua em torno de $a \in \mathbf{R}$.

Além disso, a primeira passagem do cálculo acima pode ser justificada pela seguinte proposição matematicamente trivial: para qualquer sequência delta $f_{n}$, qualquer função real integrável $h$, e qualquer constante $a>0$, temos:

$$
\lim _{n \rightarrow \infty} \int_{-\infty}^{\infty} f_{n}\left(x^{2}-a^{2}\right) h(x) d x=\lim _{n \rightarrow \infty} \int_{-\infty}^{\infty} \frac{1}{2 a}\left[f_{n}(x-a)+f_{n}(x+a)\right] h(x) d x .
$$

Para comprovar que enunciados como este traduzem com exatidão o significado das regras operacionais do Cálculo Delta, basta ler o que o próprio Dirac escreveu ([2], p. 60) sobre elas:

There are a number of elementary equations which one can write down about $\delta$ functions. These equations are essentially rules of manipulation for algebraic work involving $\delta$ functions. The meaning of any of these equations is that its two sides give equivalent results as factors in an integrand.

Esperamos ter deixado claro que as idéias matemáticas subjacentes ao Cálculo Delta, apesar de extremamente simples, não podem ser formalizadas pelo conceito de distribuição. Este fato tem passado despercebido até o momento, apesar destas idéias matemáticas estarem se mostrando úteis, há mais de 60 anos, em diversos campos da Física: "The 
pregnant messages that nature sends to the senses now fall on closed eyes and inattentive ears".

O Cálculo Delta não é um exemplo isolado: ao longo deste século, os físicos têm desenvolvido várias técnicas matemáticas relacionadas com o Cálculo Infinitesimal que não podem ser adequadamente investigadas pelos métodos tradicionais da Análise Matemática (Topologia Geral, Teoria da Medida, Análise Funcional). Algumas destas técnicas, como as integrais de trajetória de Feynman [3][4][13], são utilizadas hoje como ferramentas básicas de pesquisa em várias áreas da Física, onde podem ser encontradas espalhadas pela literatura.

Até meados do século XIX, a Análise Matemática mantinha um estreito contato com a Física, de onde proveio o próprio Cálculo Infinitesimal. Nos últimos 150 anos, entretanto, ela se ocupou basicamente em desenvolver e aprimorar seus conceitos e métodos tradicionais, que foram criados para estudar os temas em voga na primeira metade do século passado, como equações diferenciais e séries de Fourier, por exemplo. Se desejamos restabelecer o diálogo entre a Análise Matemática e a Física, devemos nos colocar inicialmente a seguinte questão: por que os métodos tradicionais da Análise são inadequados para o estudo das técnicas matemáticas relacionadas ao Cálculo Infinitesimal que os físicos têm desenvolvido ao longo deste século?

A fim de responder essa pergunta, apresentaremos agora uma releitura, um tanto pessoal, da origem e do desenvolvimento histórico dos métodos atuais da Análise Matemática:

Como sabemos, o Cálculo Infinitesimal foi originalmente formulado por Newton e Leibniz em temos de noções mal-definidas ou mesmo contraditórias, como a de infinitésimo, por exemplo. As violentas críticas ao uso de tais noções levaram então a um aumento da preocupação com o rigor formal. Observando que conceitos mal-definidos eram necessários apenas nas passagens intermediárias — os resultados finais sempre se referiam a conceitos bem estabelecidos - d'Alembert e outros matemáticos propuseram, no final do século XVIII, que os cálculos fossem justificados nos seguintes termos: concebemos um processo envolvendo objetos matemáticos variáveis, e obtemos o resultado como o limite deste processo.

A distinção clara entre o conceito de limite e a idéia de um processo subjacente foi muito importante historicamente, pois exigiu um uso intensivo, sistemático, e não-trivial da noção de tempo no discurso matemático. Até então, as expressões de natureza temporal geralmente podiam ser reinterpretadas, sem maiores dificuldades, como uma forma cmoda de referência a instâncias lógicas. Por exemplo, a frase "pode-se prolongar uma reta tanto quanto se queira" equivalia a "pode-se considerar um segmento de reta tão comprido quanto se queira", sendo que segmento de reta é uma noção atemporal. Outro exemplo: uma 
variável numérica podia ser entendida simplesmente como um número genérico, sendo que o próprio conceito de número é atemporal. Em geral, a noção de tempo estava presente na matemática apenas como um elemento linguístico auxiliar: instantes de tempo diferentes eram usados para especificar instâncias lógicas diversas.

O desenvolvimento das idéias do Cálculo Infinitesimal em termos de processos e seus limites, na primeira metade do século XIX, envolvia uma noção de tempo que não era, obviamente, o tempo físico, mas que estava presente como tal na linguagem matemática da época. Cauchy, por exemplo, utilizava ostensivamente tal noção. A sofisticação desta "linguagem temporal" permitiu um grande incremento na complexidade das construções matemáticas, mas levou também a inúmeros paradoxos e mal-entendidos, pois a antiga equivalência tácita entre instantes de tempo e instâncias lógicas era vaga demais para sustentar um uso tão intensivo de conceitos temporais no discurso matemático.

Chegamos então, em meados do século XIX, à célebre definição de limite associada ao nome de Weierstrass. A relevância desta contribuição para o pensamento matemático certamente não reside no estudo rigoroso das margens de erro, que nada mais é do que o Método da Exaustão dos antigos gregos. No contexto histórico em que foi apresentada, a definição de Weierstrass simplesmente eliminava a idéia de um processo subjacente ao limite. Ela não introduziu nenhum conceito novo, e sim retirou um conceito do formalismo do Cálculo. Juntamente com os processos, a própria noção de tempo pde então ser abolida da Análise.

Percebeu-se, um tanto subitamente, que as construções realizadas através da utilização de uma noção mal-definida de tempo na linguagem matemática podiam ser traduzidas em uma articulação lógica de idéias absolutamente atemporais. Esta percepção teve um impacto tal que acabou criando um paradigma [7] na Análise, baseado nos seguintes pressupostos metodológicos:

(i) todas as idéias relacionadas ao Cálculo Diferencial e Integral podem ser expressas satisfatoriamente em termos de limites, definidos segundo o método de Weierstrass;

(ii) não é necessário nem conveniente considerar os processos subjacentes aos limites de modo explícito, basta usar uma notação que sugira estes processos;

(iii) a noção de tempo subjacente aos processos deve ser eliminada da linguagem formal utilizada nas definições e demonstrações.

Chamaremos a visão da Análise Matemática caracterizada pela adoção destes pressupostos de paradigma weierstrasiano.

Estamos agora em condições de responder àquela pergunta sobre a inadequação dos métodos matemáticos tradicionais para o estudo das novas técnicas criadas pelos físicos ao 
longo deste século:

Os métodos tradicionais da Análise (Topologia Geral, Teoria da Medida, Análise Funcional) foram desenvolvidos nos últimos 150 anos segundo os pressupostos do paradigma weierstrasiano, enquanto os físicos continuam a pensar em termos de processos temporais, e a utilizar explicitamente a noção de tempo na sua linguagem, mesmo quando trabalhando no nível estritamente matemático, de uma forma muito semelhante àquela que vigorava na Análise na época de Cauchy.

Voltando ao exemplo do Cálculo Delta, vejamos a caracterização de Dirac para a sua função delta ([2], p. 58):

To get a picture of $\delta(x)$, take a function of the real variable $x$ which vanishes everywhere except inside a small domain, of length $\varepsilon$ say, surrounding the origin $x=0$, and which is so large inside this domain that its integral over this domain is unity. The exact shape of the function inside this domain does not matter, provided there are no unnecessarily wild variations (for example provided the function is always of order $\left.\varepsilon^{-1}\right)$. Then in the limit $\varepsilon \rightarrow 0$ this function will go over into $\delta(x)$.

Dirac utiliza explicitamente a noção de tempo na sua linguagem, a fim de descrever a função delta através de um processo (mais precisamente, de um tipo de processo: “... The exact shape of the function inside this domain does not matter ..."). As regras operacionais do Cálculo Delta também são enunciadas e demonstradas nestes termos, como propriedades da função delta. A incompatibilidade entre estes métodos e os pressupostos do paradigma weierstrasiano é evidente.

Essa incompatibilidade foi suficiente para que as concepções de Dirac fossem consideradas inaceitáveis pelos matemáticos da época, que procuraram então enquadrar suas idéias dentro do paradigma weierstrasiano: conceitualmente falando, as distribuições são limites de processos concebidos com funções. Isto fica claro ao observarmos que se $\varphi$ é uma distribuição (sobre $\mathbf{R}$ ) e $f$ uma função real integrável, então a integral:

$$
\int_{-\infty}^{\infty} \varphi(x) f(x) d x
$$

é um número real. Se as distribuições correspondessem aos processos então a integral acima seria, ela própria, um processo numérico, não um número real.

Desse ponto de vista, não é difícil constatar que as limitações operacionais do conceito de distribuição decorrem da insistência em defini-las como limites de processos com funções, e não como os processos em si, ou seja, elas refletem limitações do próprio paradigma weierstrasiano.

Os físicos sabem perfeitamente que é possível parafrasear as regras operacionais do Cálculo Delta segundo métodos tradicionais da Análise (como fizemos acima ao considerar 
sequências delta, por exemplo). O próprio Dirac nos explica ([2], p. 59) a inconveniência desta atitude:

... One could them eliminate the improper functions altogether. The use of improper functions thus does not involve any lack of rigour in the theory, but is merely a convenient notation, enabling us to express in a concise form certain relations which we could, if necessary, rewrite in a form not involving improper functions, but only in a cumbersome way which would tend to obscure the argument.

Essa alegação é perfeitamente legítima: Dirac teve bons motivos para não restringir seu pensamento matemático ao paradigma weierstrasiano, e a ampla utilização do Cálculo Delta mostra que estes motivos continuam válidos. Muitas outras construções matemáticas desenvolvidas pelos físicos ao longo deste século também não podem ser adequadamente investigadas dentro deste paradigma, pois também envolvem um uso da noção de tempo que extrapola seus pressupostos metodológicos.

Consideremos, por exemplo, as integrais de trajetória de Feynman [3][4][13], que envolvem construções semelhantes a esta ([4], p. 453):

$k(x, t)=\lim _{N \rightarrow \infty}\left(\frac{m N}{2 \pi i h t}\right)^{\frac{N}{2}} \int_{-\infty}^{\infty} \ldots \int_{-\infty}^{\infty} \exp \left\{\frac{i}{h} \sum_{j=1}^{N}\left[\frac{m N\left(x_{j+1}-x_{j}\right)^{2}}{2 t}-\frac{t V\left(x_{j+1}\right)}{N}\right]\right\} d x_{1} \cdots d x_{N-1}$.

Segundo o paradigma weierstrasiano, o processo subentendido acima não deve ser visto como um objeto matemático bem definido: apenas seu limite deve ser tratado explicitamente. Note-se que a integral múltipla, em um número crescente de variáveis, já é, ela própria, um limite de outros processos, também relegados ao limbo matemático. Devemos esperar que os métodos da Topologia Geral, da Teoria da Medida e da Análise Funcional — frutos de mais de um século de adesão da Análise a este paradigma - sejam adequados ao estudo desta construção?

Nem mesmo as idéias matemáticas triviais do Cálculo Delta podem ser adequadamente formalizadas segundo esses métodos, o que dizer então da construção matemática acima, ou daquelas estudadas pelas técnicas de renormalização da Teoria Quântica de Campos?

Além disso, muitos problemas da Física atual esperam por conceitos matemáticos que, pode-se antever, não caberão dentro do paradigma weierstrasiano. A descrição da estrutura de singularidades da Matriz S de Heisenberg talvez seja um bom exemplo.

A adesão incondicional ao paradigma weierstrasiano tem dificultado a comunicação de idéias matemáticas entre físicos teóricos e analistas ao longo deste século (o caso do Cálculo Delta é um ótimo exemplo), o que só tem reforçado o sentimento separatista entre estes últimos. Por outro lado, a mentalidade separatista tem impedido o reconhecimento de um fato cada vez mais evidente: os problemas e técnicas matemáticas que a Física 
Teórica tem proposto nas últimas décadas não poderão ser devidamente incorporadas à Análise enquanto ela restringir seus métodos àqueles do paradigma weierstrasiano. Vemos assim como o separatismo matemático e o paradigma weierstrasiano têm se realimentado e se reforçado mutuamente ao longo deste século.

Neste ponto deve estar clara a importância fundamental daquela discussão a respeito da natureza do conhecimento matemático: da perspectiva separatista, é irrelevante se o Cálculo Delta e outras técnicas matemáticas desenvolvidas pela Física nas últimas décadas podem ou não ser incorporadas à Análise. Do ponto de vista unionista, entretanto, o reconhecimento deste fato leva inevitavelmente à seguinte conclusão: devemos rever criticamente os pressupostos do paradigma weierstrasiano na Análise, a fim de restaurarmos o diálogo e a troca de idéias entre a Física e a Matemática.

Segundo a visão separatista, a Análise deve continuar aprimorando seus conceitos e métodos tradicionais, mesmo que eles se relacionem apenas com outros temas puramente matemáticos ou "aplicações seculares", como equações diferenciais e séries de Fourier. Da perspectiva unionista, contudo, vemos que cabe hoje à Matemática, em uma atitude construtiva, de intercâmbio de idéias e colaboração mútua, rever seus antigos conceitos - como o de distribuição, por exemplo - e desenvolver novos métodos que atendam às novas necessidades: este é o seu papel como parte integrante do corpo do Conhecimento Científico.

Como foi dito no início desta introdução, as idéias que apresentaremos neste trabalho refletem uma visão da matemática profundamente unionista. Uma leitura pela ótica separatista radical provavelmente lhes atribuirá pouco valor, pois não solucionamos nenhuma conjectura célebre, e também não apresentamos nenhum teorema de demonstração dificílima. Por outro lado, esperamos que esta nossa tentativa de reaproximar a Análise Matemática da Física seja bem recebida por aqueles que compartilhem conosco pelo menos um mínimo do sentimento unionista.

Partimos do princípio que o paradigma weierstrasiano, a despeito de seus grandes méritos e de sua importância histórica, está hoje esgotado: suas contribuições relevantes ao Conhecimento Científico como um todo já foram dadas há muito tempo. Defendemos a conveniência e a necessidade de se definir formalmente os processos em si, de se trabalhar explicitamente com eles, e não apenas com seus limites. Reconhecemos também que o uso indiscriminado da noção de tempo deixa margem para mal-entendidos e contradições, sendo portanto incompatível com os padrões de rigor da matemática contemporânea. Dirac, por exemplo, não deixa claro, na descrição da função delta citada acima, nem mesmo se ela é um (tipo de) processo ou seu limite: "... Then in the limit $\varepsilon \rightarrow 0$ this function will go over into $\delta(x) . "$ 
Precisamos assim de um formalismo que estimule o desenvolvimento das novas idéias relacionadas ao Cálculo Infinitesimal, sem abrir mão do rigor formal alcançado pelos métodos tradicionais da Análise. Por um lado, este formalismo deve conter uma definição matemática explícita para a idéia intuitiva de processo, e não apenas para seus limites. Por outro lado, devemos evitar que o rigor seja comprometido pela noção de tempo subjacente a esta idéia intuitiva. Dentro do espírito unionista, tal formalismo deve ser o mais simples e claro possível, a fim de não dificultar seu entendimento pelos não-especialistas. Devemos, a todo custo, evitar tecnicalidades estéreis que só contribuem para o isolamento da Matemática. A terminologia deve ser amigável, para que seu uso seja conveniente mesmo quando a Matemática comparecer apenas como ferramenta de trabalho, e não como objeto central de estudo. As notações devem estimular a invenção matemática, o pensamento indutivo, e não apenas propiciar deduções formais.

Vamos propor aqui um formalismo matemático que procura satisfazer todos os requisitos acima. Ele se baseia em um construção matemática bastante geral e relativamente simples, que pode ser desenvolvida estritamente dentro dos limites da Teoria Elementar dos Conjuntos. Apesar de sua simplicidade formal, veremos que ela é mais do que suficiente para formalizar todo o Cálculo Delta de Dirac, dentro do espírito unionista descrito acima. Tal formalismo constitui, portanto, uma abordagem mais promissora do que a atual para muitas das técnicas matemáticas que têm sido desenvolvidas pelos físicos ao longo deste século.

Conceitualmente falando, partimos de um universo de discurso, que se refere a um conjunto fixado de objetos matemáticos, e construímos um outro universo de discurso, no qual podemos nos referir a objetos virtuais, que são os processos matemáticos concebíveis com os objetos iniciais. Se entre os objetos originais tivermos, por exemplo, números e funções, então teremos, no novo universo de discurso, números virtuais e funções virtuais, que corresponderão, respectivamente, aos processos com números e funções.

Os objetos virtuais são definidos formalmente como classes de equivalência de sequências infinitas dos objetos iniciais. Construções formais deste tipo já foram utilizadas por diversos pesquisadores, em muitos contextos diferentes. Discutiremos a seguir aquelas que se relacionam mais diretamente com este trabalho, comparando os respectivos contextos ao nosso. Podemos adiantar, entretanto, que a interpretação destas classes como processos (conforme a discussão acima), balizada pelos princípios unionistas que adotamos, nos leva a idéias matemáticas originais.

A fim de tornar imediata a interpretação das classes como processos, usamos uma relação de equivalência bem simples e conhecida: duas sequências equivalem se coincidem a partir de um certo valor do índice. Esta relação foi estudada por Schmieden e Laugwitz 
[12][8][9] na construção de um formalismo que em vários pontos se assemelha ao que propomos aqui, mas que diverge deste em alguns aspectos que consideramos fundamentais. A abordagem de Schmieden e Laugwitz busca, claramente, transcender o que chamamos de paradigma weierstrasiano, e sua discussão da função delta revela interesse pelos métodos matemáticos desenvolvidos na Física, interesse este que compartilhamos.

Entretanto, Schmieden e Laugwitz se restringem, como muitos outros que propõem formalizações matemáticas para o Cálculo Delta, a demonstrar que, segundo tal definição, a função de Dirac possui a propriedade de filtragem. A regra de composição que, como vimos, representa a verdadeira essência do Cálculo Delta, não é sequer mencionada. Esta omissão deixa evidente que não está sendo levado em conta o uso que físicos efetivamente fazem do Cálculo Delta. Este é um ponto muito importante: a visão da matemática que podemos entrever no formalismo proposto por Schmieden e Laugwitz não é realmente unionista, pois o compromisso com os conceitos e métodos matemáticos tradicionais tem prioridade em relação aos problemas e soluções que vêm da Física. Como resultado, há muito pouco espaço neste formalismo para o desenvolvimento de novas técnicas e o cultivo de novas idéias.

A principal deficiência no formalismo de Schmieden e Laugwitz é que ele não oferece um método sistemático de transferirmos as propriedades dos objetos matemáticos iniciais para as classes que representam os processos concebidos com estes objetos. Como é necessário pensar sempre em termos de sequências representantes, não dispomos de um novo universo de discurso onde os processos (isto é, as classes de equivalência) possam ser tratados adequadamente. Isto fica evidente na notação utilizada, que certamente não estimula a criatividade, e chega mesmo a constituir um sério obstáculo ao leitor nãomatemático.

Não basta definirmos os processos como classes de equivalência de sequências, precisamos construir um universo de discurso apropriado para estes objetos matemáticos. Isto pode ser feito pelo desenvolvimento de técnicas que nos permitam transferir propriedades dos objetos reais (isto é, elementos do universo de discurso original) diretamente para os objetos virtuais (ou seja, os processos com objetos reais). Técnicas deste tipo são estudadas na Lógica Matemática, mas de um ponto de vista extremamente formal, bastante diferente do nosso. A construção dos objetos virtuais que realizamos aqui é conhecida na Teoria dos Modelos como o produto reduzido pelo filtro de Frechet. O artigo [14] de J. Zigmunt apresenta um ótimo apanhado sobre a origem e o desenvolvimento histórico do conceito de produto reduzido, bem como uma extensa lista de referências sobre o assunto.

Ainda no contexto da Teoria dos Modelos, é importante observar que o filtro de Frechet não é um ultra-filtro, de forma que o produto reduzido que utilizamos não é um ultra- 
produto. O formalismo matemático que estamos propondo não deve, portanto, ser confundido com a Análise Não-standard de A. Robinson [11]. A fim de evitar mal-entendidos, ressaltamos desde já que estes dois formalismos não são isomorfos: os objetos virtuais não são matematicamente equivalentes aos objetos não-standard. Como contra-exemplo: os objetos virtuais correspondentes aos números reais não formam um corpo, e nem um conjunto ordenado linearmente, ao contrário dos números reais não-standard, que constituem um corpo ordenado.

Mais importante do que a não-equivalência formal entre os objetos não-standard e os objetos virtuais é a diferença entre as ideologias matemáticas por detrás destes dois formalismos. Na introdução do livro de Robinson ([11], p. 2), por exemplo, lemos:

It is shown in this book that Leibniz' ideas can be fully vindicated and that they lead to a novel and fruitful approach to classical Analysis and to many other branches of mathematics. The key to our method is provided by the detailed analysis of the relation between mathematical languages and mathematical structures which lies at the bottom of contemporary model theory.

E mais adiante (p. 5):

One might express the hope that some branches of modern Theoretical Physics, in particular those that are afflicted with divergence problems, might be treated with profit by Non-standard Analysis. The fact that the present book contains only applications to classical Applied Mathematics most probably is a testimony to the limitations of the author and not of the method.

A Análise Não-standard foi criada a partir de um ponto de vista estritamente matemático, utilizando as sofisticadíssimas técnicas matemáticas da Lógica Simbólica. Por que devemos esperar que seus métodos possam ser "aplicados" com proveito à Física Teórica moderna?

A ideologia matemática subjacente à Análise Não-standard moldou toda sua estrutura, desde suas construções mais fundamentais até a terminologia e a notação utilizadas. Como resultado, ela não satisfaz praticamente nenhum dos requisitos unionistas que listamos acima.

A Análise Não-standard não é o formalismo mais simples possível que atende às nossas necessidades. As técnicas avançadas da Lógica Simbólica que ela utiliza são totalmente inacessíveis para os não-especialistas. Na realidade, estas técnicas são pouco acessíveis, hoje em dia, mesmo para a maioria dos matemáticos profissionais. A construção da Análise Nãostandard via Teoria dos Modelos também requer métodos sofisticados demais para os nãomatemáticos. Para demonstrar a existência de ultra-filtros, por exemplo, precisamos de um tratamento axiomático para a Teoria dos Conjuntos. Os métodos que empregamos aqui são 
muito mais simples do ponto de vista lógico-formal, sendo perfeitamente compatíveis com uma abordagem ingênua da Teoria dos Conjuntos: a técnica conjuntista mais sofisticada que utilizamos é a divisão de um conjunto por uma relação de equivalência, não precisamos do Axioma da Escolha, nem de nenhum outro postulado equivalente.

Trabalhar com ultra-filtros, quando o Filtro de Frechet é suficiente, seria ceder à tentação das tecnicalidades estéreis, que têm levado a Matemática ao isolamento. Como não é possível descrever construtivamente tais filtros, a intuição a respeito dos objetos resultantes ficaria seriamente comprometida. Um matemático talvez não se importe em não poder decidir se duas sequências dadas representam o mesmo objeto ou não, mas para um físico isto certamente faz muita diferença.

Definindo os objetos virtuais (isto é, os processos) como classes de equivalência de sequências de objetos reais segundo a relação "terminam iguais", fica muito fácil trabalhar com representantes de classe: é trivial construir objetos virtuais apresentando uma sequência específica, e também checar se duas sequências são equivalentes. Isto nos fornece uma imagem mental muito nítida do que eles sejam, ao contrário dos objetos não-standard, cuja manipulação exige um nível de abstração consideravelmente mais alto. Por sua relativa simplicidade, acreditamos que o formalismo que propomos seja uma alternativa viável mesmo para o ensino do Cálculo Elementar, tradicionalmente baseado no paradigma weierstrasiano.

primeira vista, pode parecer que também utilizamos aqui métodos da Lógica Matemática, principalmente no capítulo 1, onde apresentamos a construção em que se baseia nosso formalismo. Uma leitura mais atenta, porém, revelará que em nenhum momento fazemos uso da separação formal entre os planos sintático e semântico que caracteriza a Lógica Matemática. Todos os resultados são enunciados e demonstrados sem sequer mencionarmos uma linguagem formalizada. De fato, este trabalho não emprega nenhuma técnica da Lógica Matemática, e sua leitura não exige qualquer conhecimento prévio sobre este assunto.

A formalização dos processos como objetos matemáticos bem definidos permite que seja introduzida uma noção de infinito potencial bastante diferente dos infinitos atuais da Teoria dos Conjuntos. Este objeto virtual, que representaremos por $\infty$, possui propriedades operacionais muito mais finas do que os ordinais ou cardinais, que nunca chegaram a ser utilizados fora da "matemática pura", apesar de terem sido introduzidos há mais de um século. Alguns exemplos do que este novo conceito de infinito nos permite escrever:

$$
(\infty+1)^{2}=\infty^{2}+2 \infty+1, \quad 1+2+3+\cdots+\infty=\frac{\infty(\infty+1)}{2},
$$




$$
\begin{gathered}
\underbrace{\infty+\infty+\infty+\cdots+\infty}_{\infty \text { vezes }}=\infty . \infty=\infty^{2} \\
\frac{d}{d \xi} \xi^{\infty}=\infty \xi^{\infty-1}, \quad \int_{0}^{1 / \infty} \frac{\infty}{1+\infty^{2} \xi^{2}} d \xi=\frac{\pi}{4} \\
\frac{d}{d \xi} \int_{-\infty^{2}}^{\infty^{\xi}} e^{\tau / \infty} d \tau=e^{\infty^{\xi} / \infty} \frac{d}{d \xi}\left(\infty^{\xi}\right)=e^{\infty^{\xi-1}} \infty^{\xi} \ln \infty
\end{gathered}
$$

Acreditamos que o formalismo aqui proposto possa contribuir para o desenvolvimento das novas idéias matemáticas que há muito se fazem necessárias.

Para finalizar esta introdução, descrevemos em linhas gerais o conteúdo deste trabalho: no primeiro capítulo introduzimos a construção formal em que se baseia o processo de extensão virtual. No capítulo 2, aplicamos esta construção aos números reais, e indicamos como os números virtuais assim obtidos (entre eles temos infinitésimos e números infinitamente grandes) podem ser usados para expressar as idéias fundamentais do Cálculo Infinitesimal. Introduzimos no capítulo 3 o conceito de função virtual, e estendemos para estes objetos as noções básicas do Cálculo (continuidade, derivação, integração). No capítulo 4 mostramos como podemos formalizar o Cálculo Delta de Dirac, de uma forma simples e clara, utilizando números e funções virtuais. Concluímos com um breve epílogo, onde são discutidos os principais resultados deste trabalho, e apresentadas suas perspectivas de continuidade. 


\section{Capítulo 1}

\section{Extensões Virtuais}

Desejamos trabalhar explicitamente com processos matemáticos, sem incorrer nos malentendidos e contradições inerentes à noção subjetiva de tempo implícita em tais processos. Para tanto, partiremos neste capítulo de um universo de discurso matemático qualquer, constituído por objetos reais, e construiremos sua extensão virtual. Este novo universo de discurso é formado por objetos virtuais, que nada mais são que a formalização rigorosa dos processos concebiveis com os objetos reais. A fim de completar esta construção, tornando-a operacional, apresentaremos, também neste capítulo, uma série de conceitos e resultados relacionando objetos reais e virtuais.

\subsection{Objetos, Predicados e Funcionais}

Consideremos inicialmente um universo de discurso matemático, organizado segundo os princípios da Teoria dos Conjuntos, constituído por números, conjuntos, relações, funções e outros objetos, que vamos supor colecionados em um conjunto $\mathcal{U}$.

Vamos também supor que temos uma linguagem matemática clara para nos referirmos aos objetos do nosso universo de discurso, baseada em convenções e notações bem estabelecidas. Não será importante aqui se esta é uma linguagem natural, simbólica, ou uma mistura de ambas (que é o caso mais comum), mas estaremos supondo que sabemos distinguir um enunciado com significado matemático de um não-matemático, e, no primeiro caso, que sabemos exatamente qual é este significado.

Uma afirmação matemática clara que envolve apenas um número finito de objetos $a_{1}, a_{2}, \ldots, a_{n}$ de $\mathcal{U}$ será representada genericamente por:

$$
\mathcal{P}\left(a_{1}, a_{2}, \ldots, a_{n}\right)
$$


onde $\mathcal{P} \subset \mathcal{U}^{n}$ é o conjunto das $n$-uplas de objetos de $\mathcal{U}$ para as quais a afirmação tem sentido matemático e que, além disso, satisfazem esta afirmação.

Definição: Para cada número natural $n$, chamaremos os subconjuntos $\mathcal{P}$ de $\mathcal{U}^{n}$ de predicados $n$-ários. Estes conjuntos serão chamados genericamente de predicados finitários, ou simplesmente de predicados.

Como é habitual na Teoria dos Conjuntos, definimos formalmente uma relação entre $n$ objetos identificando-a ao conjunto das $n$-uplas de objetos que satisfazem tal relação. Os predicados assim definidos não devem ser confundidos com símbolos predicativos das linguagens formais da Lógica Matemática, que são puramente sintáticos, e cujo conteúdo semântico é sempre relativo a uma particular interpretação.

Além de afirmações matemáticas sobre nosso universo de discurso, podemos combinar seus objetos de vários modos a fim de obter um novo objeto. Vamos considerar um procedimento matemático deste tipo, que envolva apenas um número finito $n$ de objetos de $\mathcal{U}$, como uma função

$$
\mathcal{F}: \mathcal{D} \rightarrow \mathcal{U}
$$

onde $\mathcal{D} \subset \mathcal{U}^{n}$ é o conjunto das $n$-uplas de objetos de $\mathcal{U}$ para as quais o procedimento pode ser aplicado ( $\mathcal{D}$ é um predicado $n$-ário). O resultado obtido aplicando-se o procedimento $\mathcal{F}$ a $n$ objetos $a_{1}, a_{2}, \ldots, a_{n}$ de $\mathcal{U}$ será então representado por:

$$
\mathcal{F}\left(a_{1}, a_{2}, \ldots, a_{n}\right)
$$

Definição: Para cada número natural $n$, chamaremos as funções $\mathcal{F}: \mathcal{D} \rightarrow \mathcal{U}$ com $\mathcal{D} \subset \mathcal{U}^{n}$ de funcionais $n$-ários, e diremos que o predicado $n$-ário $\mathcal{D}$ é o seu domínio. Essas funções serão chamadas genericamente de funcionais finitários, ou simplesmente de funcionais.

Os funcionais assim definidos não devem ser confundidos com símbolos funcionais da Lógica Matemática, que são puramente sintáticos, e cujo conteúdo semântico é sempre relativo a uma particular interpretação.

Adotaremos, daqui por diante, a seguinte convenção: quando o domínio de um funcional $n$-ário $\mathcal{F}$ não for mencionado explicitamente, deve-se subentender que ele é o predicado $n$-ário $\mathcal{D}$ dado por:

$$
\mathcal{D}\left(a_{1}, a_{2}, \ldots, a_{n}\right): \quad \mathcal{F}\left(a_{1}, a_{2}, \ldots, a_{n}\right) \text { pode ser determinado }
$$

univocamente e é um objeto de $\mathcal{U}$. 


\subsection{Objetos, Predicados e Funcionais Virtuais}

É imediata a interpretação de uma sequência infinita de objetos de $\mathcal{U}$ como um processo envolvendo estes objetos. Entretanto, será conveniente considerar que duas sequências que diferem apenas em um número finito de termos representam o mesmo processo. A fim de formalizar esta idéia:

DefiniÇÃo: Diremos que duas sequências infinitas de objetos de $\mathcal{U}$ terminam iguais quando elas coincidem a partir de algum valor do índice. Em outras palavras, as sequências $\left(a_{i}\right)=\left(a_{1}, a_{2}, \ldots\right)$ e $\left(b_{i}\right)=\left(b_{1}, b_{2}, \ldots\right)$ terminam iguais quando existe $n \in \mathbf{N}$ tal que $i>n$ implique em $a_{i}=b_{i}$.

É fácil verificar que essa é uma relação de equivalência entre sequências de objetos de $\mathcal{U}$, assim:

DEFINiÇÃo: As classes de equivalência pela relação "terminam iguais" serão chamadas de objetos virtuais, em oposição aos objetos reais que constituem $\mathcal{U}$. Além disso, representaremos o conjunto de todos os objetos virtuais por $\overline{\mathcal{U}}$.

Dessa forma, cada processo fica formalmente definido como um objeto virtual, isto é, como uma classe de equivalência de sequências de objetos reais segundo a relação "terminam iguais". Representaremos a classe de equivalência de uma sequência $\left(a_{i}\right)$ por $\left\langle a_{i}\right\rangle$, de forma que $\left\langle a_{i}\right\rangle=\left\langle b_{i}\right\rangle$ se e somente se $\left(a_{i}\right)$ e $\left(b_{i}\right)$ terminarem iguais.

Adotaremos, deste ponto em diante, a seguinte convenção notacional: sempre que representarmos objetos por letras, usaremos letras latinas para os objetos reais, e letras gregas para os virtuais.

Na seção anterior, representamos uma afirmação matemática genérica envolvendo $n$ objetos reais de $\mathcal{U}$ pelo predicado $n$-ário correspondente, isto é, pelo subconjunto de $\mathcal{U}^{n}$ das $n$-uplas que satisfazem esta afirmação. Analogamente:

DefiniÇÃo: Para cada número natural $n$, chamaremos os subconjuntos de $\overline{\mathcal{U}}^{n}$ de predicados virtuais $n$-ários.

Assim, uma afirmação matemática clara envolvendo $n$ objetos virtuais está sempre associada ao predicado virtual $n$-ário correspondente, isto é, ao conjunto das $n$-uplas de objetos virtuais para as quais esta afirmação tem sentido matemático e é verdadeira.

Na seção anterior, uma operação matemática genérica envolvendo $n$ objetos de $\mathcal{U}$ foi representada pelo funcional $n$-ário correspondente. De forma análoga:

DEFINIÇÃO: Para cada número natural $n$, chamaremos as funções definidas de subconjuntos de $\overline{\mathcal{U}}^{n}$ a valores em $\overline{\mathcal{U}}$ de funcionais virtuais $n$-ários.

Desse modo, uma operação matemática bem definida, envolvendo $n$ objetos virtuais está sempre associada a um funcional virtual $n$-ário. 


\subsection{Transferência de Objetos, Predicados e Funcionais}

A definição seguinte associa a cada objeto real de $\mathcal{U}$ um objeto virtual de $\overline{\mathcal{U}}$. DEFINIÇÃo: Se a é um objeto real qualquer, definimos sua extensão virtual $\bar{a}$ como sendo a classe de equivalência da sequência constante $(a, a, a, \ldots)$, isto é:

$$
\bar{a}=\langle a, a, a, \ldots\rangle
$$

Vamos também associar a cada predicado $n$-ário $\mathcal{P}$ um predicado virtual $n$-ário $\overline{\mathcal{P}}$ : se $\alpha_{1}, \alpha_{2}, \ldots, \alpha_{n}$ são $n$ objetos virtuais:

$$
\alpha_{j}=\left\langle a_{j}^{1}, a_{j}^{2}, a_{j}^{3}, \ldots\right\rangle \quad(j=1, \ldots, n)
$$

então diremos que a afirmação matemática

$$
\overline{\mathcal{P}}\left(\alpha_{1}, \alpha_{2}, \ldots, \alpha_{n}\right)
$$

é verdadeira quando existir $k \in \mathbf{N}$ tal que, para todo $i>k$, temos

$$
\mathcal{P}\left(a_{1}^{i}, a_{2}^{i}, \ldots, a_{n}^{i}\right)
$$

É fácil verificar que esta definição é independente da escolha das sequências representantes dos objetos virtuais $\alpha_{1}, \alpha_{2}, \ldots, \alpha_{n}$, pois $\mathcal{P}$ é uma predicado finitário.

DEFINIÇÃO: Chamaremos o predicado virtual $\overline{\mathcal{P}}$ definido acima de extensão virtual do predicado $\mathcal{P}$.

Vamos agora associar a cada funcional $n$-ário

$$
\mathcal{F}: \mathcal{D} \rightarrow \mathcal{U}, \quad \text { com } \quad \mathcal{D} \subset \mathcal{U}^{n},
$$

um funcional virtual $n$-ário $\overline{\mathcal{F}}$ definido na extensão virtual de $\mathcal{D}$ :

$$
\overline{\mathcal{F}}: \overline{\mathcal{D}} \rightarrow \overline{\mathcal{U}}
$$

Para tanto, tomemos $n$ objetos virtuais $\alpha_{1}, \alpha_{2}, \ldots, \alpha_{n}$ tais que

$$
\overline{\mathcal{D}}\left(\alpha_{1}, \alpha_{2}, \ldots, \alpha_{n}\right) \text {. }
$$

Se

$$
\alpha_{j}=\left\langle a_{j}^{1}, a_{j}^{2}, a_{j}^{3}, \ldots\right\rangle \quad(j=1, \ldots, n)
$$


então existe $k \in \mathbf{N}$ tal que, para todo $i>k$ :

$$
\mathcal{D}\left(a_{1}^{i}, a_{2}^{i}, \ldots, a_{n}^{i}\right)
$$

Portanto, para todo $i>k$, existe um e um único objeto $b^{i}$ dado por:

$$
b^{i}=\mathcal{F}\left(a_{1}^{i}, a_{2}^{i}, \ldots, a_{n}^{i}\right) .
$$

Tomando agora $k$ objetos arbitrários $b_{1}, b_{2}, \ldots, b_{k}$, construímos a sequência:

$$
\left(b_{1}, b_{2}, \ldots, b_{k}, b^{k+1}, b^{k+2}, \ldots\right)
$$

Como $\mathcal{F}$ é um funcional finitário, é fácil verificar que a classe de equivalência $\beta \in \overline{\mathcal{U}}$ desta sequência (pela relação "terminam iguais") não depende da escolha das sequências representantes dos objetos virtuais $\alpha_{1}, \alpha_{2}, \ldots, \alpha_{n}$, nem tampouco dos $k$ objetos reais $b_{1}, b_{2}, \ldots, b_{k}$ tomados arbitrariamente .

DefiniçÃo: Chamaremos o funcional virtual $\overline{\mathcal{F}}$ que leva os $n$ objetos virtuais $\alpha_{1}, \ldots$ $\ldots, \alpha_{n}$ no objeto virtual

$$
\overline{\mathcal{F}}\left(\alpha_{1}, \alpha_{2}, \ldots, \alpha_{n}\right)=\beta
$$

definido acima de extensão virtual do funcional $\mathcal{F}$.

\subsection{O Teorema da Extensão Virtual}

Estamos supondo que temos uma linguagem matemática clara para nos referirmos aos objetos do nosso universo de discurso $\mathcal{U}$. As afirmações matemáticas sobre $\mathcal{U}$ são enunciadas combinando-se alguns objetos, predicados e funcionais de $\mathcal{U}$, segundo construções lógicas previamente estabelecidas, de acordo com as regras gramaticais desta linguagem.

Estaremos interessados, no que segue, nas afirmações matemáticas sobre os objetos de $\mathcal{U}$ que não envolvem outras construções lógicas além das seguintes:

(i) predicações;

(ii) igualdades;

(iii) conjunções;

(iv) quantificações existenciais;

(v) quantificações exclusivas;

(vi) quantificações universais.

A forma exata dos enunciados que contêm estas construções depende da linguagem matemática que estamos utilizando, mas seu significado não depende: quando usadas no 
escopo das variáveis $x_{1}, x_{2}, \ldots, x_{n}$, estes enunciados são logicamente equivalentes aos seguintes:

(i) Em uma predicação, afirmamos que:

$$
\mathcal{P}\left(x_{1}, x_{2}, \ldots, x_{n}\right)
$$

onde $\mathcal{P}$ é um predicado $n$-ário.

(ii) Uma igualdade é uma afirmação do tipo:

$$
\mathcal{F}\left(x_{1}, x_{2}, \ldots, x_{n}\right)=\mathcal{G}\left(x_{1}, x_{2}, \ldots, x_{n}\right)
$$

onde $\mathcal{F}$ e $\mathcal{G}$ são dois funcionais $n$-ários.

(iii) Em uma conjunção afirmamos que duas outras asserções são ambas verdadeiras:

$$
\mathcal{P}\left(x_{1}, x_{2}, \ldots, x_{n}\right) \text { e } \mathcal{Q}\left(x_{1}, x_{2}, \ldots, x_{n}\right)
$$

onde $\mathcal{P}$ e $\mathcal{Q}$ são dois predicados $n$-ários.

(iv) Uma quantificação existencial é uma afirmação do tipo:

$$
\text { Existe } y \in \mathcal{U} \text { tal que } \mathcal{P}\left(y, x_{1}, x_{2}, \ldots, x_{n}\right) \text {, }
$$

onde $\mathcal{P}$ é um predicado $(n+1)$-ário.

(v) Uma quantificação exclusiva é uma afirmação da forma:

Existe um e um único $y \in \mathcal{U}$ tal que $\mathcal{P}\left(y, x_{1}, x_{2}, \ldots, x_{n}\right)$, onde $\mathcal{P}$ é um predicado $(n+1)$-ário.

(vi) Uma quantificação universal é uma afirmação do tipo:

$$
\begin{aligned}
& \text { Para toda } k \text {-upla }\left(y_{1}, \ldots, y_{k}\right) \in \mathcal{U}^{k} \text { tal que } \mathcal{P}\left(y_{1}, \ldots, y_{k}\right) \\
& \text { temos } \mathcal{Q}\left(y_{1}, \ldots, y_{k}, x_{1}, \ldots, x_{n}\right)
\end{aligned}
$$

onde $\mathcal{P}$ é um predicado $k$-ário e $\mathcal{Q}$ um predicado $(n+k)$-ário. É importante observar que a condição $\mathcal{P}\left(y_{1}, \ldots, y_{k}\right)$ acima não envolve as variáveis $x_{1}, \ldots, x_{n}$, o motivo desta restrição ficará claro na próxima seção.

DEFINIÇÃO: Chamaremos as construções lógicas dos seis tipos acima de admissiveis. 
Consideremos agora uma afirmação matemática sobre $\mathcal{U}$, cujo enunciado mencione apenas:

(a) os objetos específicos $a_{1}, a_{2}, \ldots, a_{l}$;

(b) os predicados $\mathcal{P}_{1}, \mathcal{P}_{2}, \ldots, \mathcal{P}_{p}$; e

(c) os funcionais $\mathcal{F}_{1}, \mathcal{F}_{2}, \ldots, \mathcal{F}_{f}$.

Podemos fazer um afirmação matemática sobre os objetos virtuais de $\overline{\mathcal{U}}$ substituindo:

(a) cada ocorrência do objeto específico $a_{i}$ pela sua respectiva extensão virtual $\overline{a_{i}}$, para $i=1, \ldots, l$;

(b) cada ocorrência do predicado $\mathcal{P}_{i}$ pela sua respectiva extensão virtual $\overline{\mathcal{P}_{i}}$, para $i=1, \ldots, p$;

(c) cada ocorrência do funcional $\mathcal{F}_{i}$ pela sua respectiva extensão virtual $\overline{\mathcal{F}_{i}}$, para $i=1, \ldots, f$.

DEFINIÇÃO: Chamaremos o enunciado matemático sobre os objetos virtuais de $\overline{\mathcal{U}}$ assim obtido de extensão virtual da afirmação original sobre $\mathcal{U}$.

Construir a extensão virtual de uma afirmação sobre objetos de $\mathcal{U}$ significa, conceitualmente falando, estender esta afirmação para os processos com estes objetos. É fácil ver que a extensão virtual de uma afirmação matemática clara sobre $\mathcal{U}$ é sempre uma afirmação matemática clara sobre $\overline{\mathcal{U}}$. Chegamos assim ao resultado central deste capítulo:

Teorema da Extensão Virtual: Se uma afirmação matemática clara sobre $\mathcal{U}$ envolve apenas construções lógicas admissíveis, em número finito, então sua extensão virtual será verdadeira se e somente se ela própria for verdadeira.

\subsection{Prova do Teorema da Extensão Virtual}

A fim de demonstrarmos o TEV (Teorema da Extensão Virtual), introduzimos abaixo algumas definições e provamos alguns resultados preliminares. Daqui por diante, usaremos o símbolo ' $\forall$ ' como abreviação de 'para todo' (quantificador lógico universal), o símbolo ' $\exists$ ' significará 'existe' (quantificador existencial), e ' $\exists$ !' será uma abreviação de 'existe um e um único' (quantificador existencial exclusivo). Por exemplo, a afirmação:

$$
\text { existe } k \in \mathbf{N} \text { tal que, para todo } i>k \text {, temos } \mathcal{P}\left(x_{i}\right)
$$

será abreviada como:

$$
\exists k \in \mathbf{N}, \forall i>k, \mathcal{P}\left(x_{i}\right) .
$$


DefiniÇÃo: Para todo $n \in \mathbf{N}$, e para $i=1, \ldots, n$, denotaremos por $\mathcal{S}_{i}^{n}: \mathcal{U}^{n} \rightarrow \mathcal{U}$ o funcional $n$-ário que seleciona a $i$-ésima entrada de cada $n$-upla:

$$
\mathcal{S}_{i}^{n}\left(x_{1}, \ldots, x_{n}\right)=x_{i}
$$

Estes funcionais $\mathcal{S}_{i}^{n}$ serão chamados de seletores.

LEma: Para todo $n \in \mathbf{N}$, e para $i=1, \ldots, n$, temos:

$$
\overline{\mathcal{S}_{i}^{n}}\left(\xi_{1}, \ldots, \xi_{n}\right)=\xi_{i} .
$$

Prova: Se $\xi_{j}=\left\langle x_{j}^{1}, x_{j}^{2}, \ldots\right\rangle(j=1, \ldots, n) \mathrm{e}$

$$
\overline{\mathcal{S}_{i}^{n}}\left(\xi_{1}, \ldots, \xi_{n}\right)=\left\langle y^{1}, y^{2}, \ldots\right\rangle,
$$

então

$$
\exists k \in \mathbf{N}, \forall l>k, \mathcal{S}_{i}^{n}\left(x_{1}^{l}, \ldots, x_{n}^{l}\right)=y^{l},
$$

$\log O$

$$
\exists k \in \mathbf{N}, \forall l>k, x_{i}^{l}=y^{l},
$$

portanto $\left\langle y^{1}, y^{2}, \ldots\right\rangle=\xi_{i}$.

DEFINIÇÃo: Denotaremos por $\mathcal{I} \subset \mathcal{U}^{2}$ o predicado binário de igualdade:

$$
\mathcal{I}(x, y) \quad \text { se e somente se } \quad x=y \text {. }
$$

LEMA: Para quaisquer objetos virtuais $\xi$ e $v$, temos:

$$
\overline{\mathcal{I}}(\xi, v) \quad \text { se e somente se } \quad \xi=v \text {. }
$$

Prova: Se $\xi=\left\langle x_{1}, x_{2}, \ldots\right\rangle$ e $v=\left\langle y_{1}, y_{2}, \ldots\right\rangle$ então as afirmações seguintes são equivalentes:

$$
\begin{aligned}
& \overline{\mathcal{I}}(\xi, v) \\
& \exists k \in \mathbf{N}, \forall i>k, \mathcal{I}\left(x_{i}, y_{i}\right) \\
& \exists k \in \mathbf{N}, \forall i>k, x_{i}=y_{i} \\
& \xi=v .
\end{aligned}
$$

Vamos definir agora uma série de operações envolvendo objetos, predicado e funcionais:

DefiniÇÃo: Se $\mathcal{F}$ é um funcional $(n+1)$-ário, e a um objeto qualquer de $\mathcal{U}$, indicaremos por $(\mathcal{F} a)$ o funcional $n$-ário dado por:

$$
(\mathcal{F} a)\left(x_{1}, \ldots, x_{n}\right)=\mathcal{F}\left(a, x_{1}, \ldots, x_{n}\right) .
$$


Temos assim uma operação que, aplicada sobre um funcional $\mathcal{F}$ e um objeto $a$, nos fornece um novo funcional $(\mathcal{F} a)$. Esta operação, que chamaremos de avaliação parcial, será também aplicada, de forma análoga, sobre funcionais e objetos virtuais, fornecendo então um funcional virtual.

DefiniçÃo: Se $\mathcal{F}$ é um funcional $k$-ário e $\mathcal{G}_{1}, \ldots, \mathcal{G}_{k}$ são $k$ funcionais $n$-ários, representaremos por $\mathcal{F} \circ\left(\mathcal{G}_{1}, \ldots, \mathcal{G}_{k}\right)$ o funcional $n$-ário dado por:

$$
\left[\mathcal{F} \circ\left(\mathcal{G}_{1}, \ldots, \mathcal{G}_{k}\right)\right]\left(x_{1}, \ldots, x_{n}\right)=\mathcal{F}\left[\mathcal{G}_{1}\left(x_{1}, \ldots, x_{n}\right), \ldots, \mathcal{G}_{k}\left(x_{1}, \ldots, x_{n}\right)\right]
$$

A operação assim definida, que nos permite obter um novo funcional a partir de outros, será chamada de composição funcional. Ela também será aplicada, de forma análoga, sobre funcionais virtuais, fornecendo então um funcional virtual.

DefiniçÃo: Se $\mathcal{P}$ é um predicado $k$-ário e $\mathcal{F}_{1}, \ldots, \mathcal{F}_{k}$ são $k$ funcionais $n$-ários, indicaremos por $\mathcal{P} \circ\left(\mathcal{F}_{1}, \ldots, \mathcal{F}_{k}\right)$ o predicado $n$-ário dado por:

$$
\begin{gathered}
{\left[\mathcal{P} \circ\left(\mathcal{F}_{1}, \ldots, \mathcal{F}_{k}\right)\right]\left(x_{1}, \ldots, x_{n}\right) \quad \text { se e somente se }} \\
\mathcal{P}\left[\mathcal{F}_{1}\left(x_{1}, \ldots, x_{n}\right), \ldots, \mathcal{F}_{k}\left(x_{1}, \ldots, x_{n}\right)\right]
\end{gathered}
$$

A operação assim definida, que parte de um predicado e alguns funcionais, e nos fornece um novo predicado, será chamada de composição predicativa. Ela será também aplicada, de forma análoga, sobre predicados e funcionais virtuais, fornecendo então um predicado virtual.

DefiniÇÃo: Se $\mathcal{P}$ e $\mathcal{Q}$ são dois predicados $n$-ários quaisquer, representaremos por $(\mathcal{P}$ e $\mathcal{Q})$ o predicado $n$-ário dado por:

$$
\begin{aligned}
& (\mathcal{P} \text { e } \mathcal{Q})\left(x_{1}, \ldots, x_{n}\right) \quad \text { se e somente se } \\
& \mathcal{P}\left(x_{1}, \ldots, x_{n}\right) \quad \text { e } \quad \mathcal{Q}\left(x_{1}, \ldots, x_{n}\right) .
\end{aligned}
$$

Essa forma de se construir um predicado a partir de dois outros será chamada de operação de conjunção. Ela também será aplicada, de forma análoga, sobre dois predicados virtuais, fornecendo então um novo predicado virtual.

DefiniçÃo: Se $\mathcal{P}$ é um predicado $(n+1)$-ário qualquer, indicaremos por $(\exists$ tq $\mathcal{P})$ o predicado $n$-ário dado por:

$$
\begin{gathered}
(\exists \operatorname{tq} \mathcal{P})\left(x_{1}, \ldots, x_{n}\right) \quad \text { se e somente se } \\
\exists y \in \mathcal{U} \text { tal que } \mathcal{P}\left(y, x_{1}, \ldots, x_{n}\right) .
\end{gathered}
$$


Essa operação, que nos fornece um predicado a partir de outro, será chamada de quantificação existencial parcial. Ela será também aplicada, de forma análoga, sobre predicados virtuais.

DEFINIÇÃo: Se $\mathcal{P}$ é um predicado $(n+1)$-ário qualquer, indicaremos por $(\exists$ ! tq $\mathcal{P})$ o predicado $n$-ário dado por:

$$
\begin{gathered}
(\exists ! \operatorname{tq} \mathcal{P})\left(x_{1}, \ldots, x_{n}\right) \quad \text { se e somente se } \\
\exists ! y \in \mathcal{U} \text { tal que } \mathcal{P}\left(y, x_{1}, \ldots, x_{n}\right) .
\end{gathered}
$$

Essa operação, que nos fornece um predicado a partir de outro, será chamada de quantificação exclusiva parcial. Ela também será aplicada, de forma análoga, sobre predicados virtuais.

DefiniçÃo: Se $\mathcal{P}$ é um predicado $k$-ário qualquer, e $\mathcal{Q}$ um predicado $(n+k)$-ário qualquer, indicaremos por $(\forall \mathcal{P} \mathcal{Q})$ o predicado $n$-ário dado por:

$$
\begin{gathered}
(\forall \mathcal{P} \mathcal{Q})\left(x_{1}, \ldots, x_{n}\right) \quad \text { se e somente se } \\
\forall\left(y_{1}, \ldots, y_{k}\right) \in \mathcal{U}^{k} \text { tal que } \mathcal{P}\left(y_{1}, \ldots, y_{k}\right), \text { temos } \mathcal{Q}\left(y_{1}, \ldots, y_{k}, x_{1}, \ldots, x_{n}\right) .
\end{gathered}
$$

Essa operação que nos fornece um predicado a partir de dois outros será chamada de quantificação universal parcial. Ela será também aplicada, de forma análoga, sobre dois predicados virtuais, fornecendo então um novo predicado virtual.

LEMA: Com as notações definidas acima, temos que:

(i) $\overline{(\mathcal{F} a)}=(\overline{\mathcal{F}} \bar{a})$;

(ii) $\overline{\mathcal{F} \circ\left(\mathcal{G}_{1}, \ldots, \mathcal{G}_{k}\right)}=\overline{\mathcal{F}} \circ\left(\overline{\mathcal{G}_{1}}, \ldots, \overline{\mathcal{G}_{k}}\right)$;

(iii) $\overline{\mathcal{P} \circ\left(\mathcal{F}_{1}, \ldots, \mathcal{F}_{k}\right)}=\overline{\mathcal{P}} \circ\left(\overline{\mathcal{F}_{1}}, \ldots, \overline{\mathcal{F}_{k}}\right)$;

(iv) $\overline{(\mathcal{P} \text { e } \mathcal{Q})}=(\overline{\mathcal{P}}$ e $\overline{\mathcal{Q}})$;

(v) $\overline{(\exists \operatorname{tq} \mathcal{P})}=(\exists \operatorname{tq} \overline{\mathcal{P}})$;

(vi) $\overline{(\exists ! \operatorname{tq} \mathcal{P})}=(\exists$ ! tq $\overline{\mathcal{P}})$;

(vii) $\overline{(\forall \mathcal{P} \mathcal{Q})}=(\forall \overline{\mathcal{P}} \overline{\mathcal{Q}})$.

Prova: Em todos os itens abaixo, representaremos uma $n$-upla arbitrária de objetos virtuais por $\left(\xi_{1}, \ldots, \xi_{n}\right)$, onde:

$$
\xi_{j}=\left\langle x_{j}^{1}, x_{j}^{2}, \ldots\right\rangle \quad(j=1, \ldots, n) .
$$

(i) $\mathrm{Se}$

$$
\overline{(\mathcal{F} a)}\left(\xi_{1}, \ldots, \xi_{n}\right)=\left\langle y^{1}, y^{2}, \ldots\right\rangle
$$


então

$$
\exists k \in \mathbf{N}, \forall i>k,(\mathcal{F} a)\left(x_{1}^{i}, \ldots, x_{n}^{i}\right)=y^{i},
$$

$\log O$

$$
\exists k \in \mathbf{N}, \forall i>k, \mathcal{F}\left(a, x_{1}^{i}, \ldots, x_{n}^{i}\right)=y^{i},
$$

portanto

$$
\overline{\mathcal{F}}\left(\bar{a}, \xi_{1}, \ldots, \xi_{n}\right)=\left\langle y^{1}, y^{2}, \ldots\right\rangle
$$

Ou seja:

$$
\overline{(\mathcal{F} a)}\left(\xi_{1}, \ldots, \xi_{n}\right)=(\overline{\mathcal{F}} \bar{a})\left(\xi_{1}, \ldots, \xi_{n}\right)
$$

para qualquer $n$-upla $\left(\xi_{1}, \ldots, \xi_{n}\right)$ de objetos virtuais.

(ii) Se

$$
\overline{\mathcal{G}_{l}}\left(\xi_{1}, \ldots, \xi_{n}\right)=\left\langle y_{l}^{1}, y_{l}^{2}, \ldots\right\rangle \quad(l=1, \ldots, k)
$$

então

$$
\exists m_{l} \in \mathbf{N}, \forall i>m_{l}, \mathcal{G}_{l}\left(x_{1}^{i}, \ldots, x_{n}^{i}\right)=y_{l}^{i} \quad(l=1, \ldots, k)
$$

Além disso, se

$$
\overline{\left[\mathcal{F} \circ\left(\mathcal{G}_{1}, \ldots, \mathcal{G}_{k}\right)\right]}\left(\xi_{1}, \ldots, \xi_{n}\right)=\left\langle z^{1}, z^{2}, \ldots\right\rangle
$$

então

$$
\exists m \in \mathbf{N}, \forall i>m,\left[\mathcal{F} \circ\left(\mathcal{G}_{1}, \ldots, \mathcal{G}_{k}\right)\right]\left(x_{1}^{i}, \ldots, x_{n}^{i}\right)=z^{i}
$$

Tomando agora $r=\operatorname{máx}\left\{m, m_{1}, \ldots, m_{k}\right\}$, obtemos:

$$
\exists r \in \mathbf{N}, \forall i>r, \mathcal{F}\left(y_{1}^{i}, \ldots, y_{k}^{i}\right)=z^{i},
$$

$\log O$

$$
\overline{\mathcal{F}}\left[\overline{\mathcal{G}_{1}}\left(\xi_{1}, \ldots, \xi_{n}\right), \ldots, \overline{\mathcal{G}_{k}}\left(\xi_{1}, \ldots, \xi_{n}\right)\right]=\left\langle z^{1}, z^{2}, \ldots\right\rangle
$$

Portanto

$$
\overline{\left[\mathcal{F} \circ\left(\mathcal{G}_{1}, \ldots, \mathcal{G}_{k}\right)\right]}\left(\xi_{1}, \ldots, \xi_{n}\right)=\left[\overline{\mathcal{F}} \circ\left(\overline{\mathcal{G}_{1}}, \ldots, \overline{\mathcal{G}_{k}}\right)\right]\left(\xi_{1}, \ldots, \xi_{n}\right)
$$

para qualquer $n$-upla $\left(\xi_{1}, \ldots, \xi_{n}\right)$ de objetos virtuais. 
(iii) Se

$$
\overline{\mathcal{F}_{l}}\left(\xi_{1}, \ldots, \xi_{n}\right)=\left\langle y_{l}^{1}, y_{l}^{2}, \ldots\right\rangle \quad(l=1, \ldots, k)
$$

então

$$
\exists m_{l} \in \mathbf{N}, \forall i>m_{l}, \mathcal{F}_{l}\left(x_{1}^{i}, \ldots, x_{n}^{i}\right)=y_{l}^{i} \quad(l=1, \ldots, k) .
$$

Além disso, as seguintes afirmações são equivalentes:

$$
\begin{aligned}
& \overline{\left[\mathcal{P} \circ\left(\mathcal{F}_{1}, \ldots, \mathcal{F}_{k}\right)\right]}\left(\xi_{1}, \ldots, \xi_{n}\right) \\
& \exists m \in \mathbf{N}, \forall i>m,\left[\mathcal{P} \circ\left(\mathcal{F}_{1}, \ldots, \mathcal{F}_{k}\right)\right]\left(x_{1}^{i}, \ldots, x_{n}^{i}\right) .
\end{aligned}
$$

Assim, se essa afirmação $(*)$ for verdadeira, tomamos $r=\operatorname{máx}\left\{m, m_{1}, \ldots, m_{k}\right\}$, e concluímos que:

$$
\exists r \in \mathbf{N}, \forall i>r, \mathcal{P}\left(y_{1}^{i}, \ldots, y_{k}^{i}\right) \quad(* *) .
$$

Por outro lado, se esta afirmação $(* *)$ for verdadeira, então fazemos

$$
m=\operatorname{máx}\left\{r, m_{1}, \ldots, m_{k}\right\},
$$

e concluímos que $(*)$ também é verdadeira.

Para finalizar a prova deste item, basta observar que $(* *)$ é equivalente a

$$
\overline{\mathcal{P}}\left[\overline{\mathcal{F}_{1}}\left(\xi_{1}, \ldots, \xi_{n}\right), \ldots, \overline{\mathcal{F}_{k}}\left(\xi_{1}, \ldots, \xi_{n}\right)\right],
$$

e portanto:

$$
\overline{\left[\mathcal{P} \circ\left(\mathcal{F}_{1}, \ldots, \mathcal{F}_{k}\right)\right]}\left(\xi_{1}, \ldots, \xi_{n}\right) \quad \text { se e somente se } \quad\left[\overline{\mathcal{P}} \circ\left(\overline{\mathcal{F}_{1}}, \ldots, \overline{\mathcal{F}_{k}}\right)\right]\left(\xi_{1}, \ldots, \xi_{n}\right),
$$

para qualquer $n$-upla $\left(\xi_{1}, \ldots, \xi_{n}\right)$ de objetos virtuais.

(iv) Equivalem:

$$
\begin{aligned}
& \overline{(\mathcal{P} \text { e } \mathcal{Q})}\left(\xi_{1}, \ldots, \xi_{n}\right) \\
& \exists k \in \mathbf{N}, \forall i>k,(\mathcal{P} \text { e } \mathcal{Q})\left(x_{1}^{i}, \ldots, x_{n}^{i}\right) \\
& \exists k \in \mathbf{N}, \forall i>k, \mathcal{P}\left(x_{1}^{i}, \ldots, x_{n}^{i}\right) \text { e } \mathcal{Q}\left(x_{1}^{i}, \ldots, x_{n}^{i}\right)
\end{aligned}
$$

Esta última afirmação é equivalente a

$$
\begin{gathered}
\exists k_{1} \in \mathbf{N}, \forall i>k_{1}, \mathcal{P}\left(x_{1}^{i}, \ldots, x_{n}^{i}\right) \\
\mathrm{e} \\
\exists k_{2} \in \mathbf{N}, \forall i>k_{2}, \mathcal{Q}\left(x_{1}^{i}, \ldots, x_{n}^{i}\right),
\end{gathered}
$$

que, por sua vez, equivale a

$$
\overline{\mathcal{P}}\left(\xi_{1}, \ldots, \xi_{n}\right) \text { e } \overline{\mathcal{Q}}\left(\xi_{1}, \ldots, \xi_{n}\right)
$$

Portanto:

$$
\overline{(\mathcal{P} \text { e } \mathcal{Q})}\left(\xi_{1}, \ldots, \xi_{n}\right) \quad \text { se e somente se } \quad(\overline{\mathcal{P}} \text { e } \overline{\mathcal{Q}})\left(\xi_{1}, \ldots, \xi_{n}\right),
$$

para qualquer $n$-upla $\left(\xi_{1}, \ldots, \xi_{n}\right)$ de objetos virtuais. 
(v) As seguintes afirmações são equivalentes:

$$
\begin{aligned}
& \overline{(\exists \operatorname{tg} \mathcal{P})}\left(\xi_{1}, \ldots, \xi_{n}\right) \\
& \exists k \in \mathbf{N}, \forall i>k,(\exists \operatorname{tq} \mathcal{P})\left(x_{1}^{i}, \ldots, x_{n}^{i}\right) \\
& \exists k \in \mathbf{N}, \forall i>k, \exists y \in \mathcal{U} \text { tal que } \mathcal{P}\left(y, x_{1}^{i}, \ldots, x_{n}^{i}\right)
\end{aligned}
$$

Esta última afirmação equivale a dizer:

Existe uma sequência $\left(y^{1}, y^{2}, \ldots\right)$ de objetos de $\mathcal{U}$ para a qual $\exists k \in \mathbf{N}, \forall i>k, \mathcal{P}\left(y^{i}, x_{1}^{i}, \ldots, x_{n}^{i}\right)$.

Tomando agora $v=\left\langle y^{1}, y^{2}, \ldots\right\rangle$, vemos que isto é o mesmo que afirmar:

$$
\exists v \in \overline{\mathcal{U}} \text { tal que } \overline{\mathcal{P}}\left(v, \xi_{1}, \ldots, \xi_{n}\right) .
$$

Portanto:

$$
\overline{(\exists \operatorname{tq} \mathcal{P})}\left(\xi_{1}, \ldots, \xi_{n}\right) \quad \text { se e somente se } \quad(\exists \operatorname{tq} \overline{\mathcal{P}})\left(\xi_{1}, \ldots, \xi_{n}\right)
$$

para qualquer $n$-upla $\left(\xi_{1}, \ldots, \xi_{n}\right)$ de objetos virtuais.

(vi) Equivalem:

$$
\begin{aligned}
& \overline{(\exists ! \operatorname{tq} \mathcal{P})}\left(\xi_{1}, \ldots, \xi_{n}\right) \\
& \exists k \in \mathbf{N}, \forall i>k, \quad(\exists ! \operatorname{tq} \mathcal{P})\left(x_{1}^{i}, \ldots, x_{n}^{i}\right) \\
& \exists k \in \mathbf{N}, \forall i>k, \exists ! y \in \mathcal{U} \text { tal que } \mathcal{P}\left(y, x_{1}^{i}, \ldots, x_{n}^{i}\right),
\end{aligned}
$$

e esta última afirmação é equivalente a

$$
\exists ! v \in \overline{\mathcal{U}} \text { tal que } \overline{\mathcal{P}}\left(v, \xi_{1}, \ldots, \xi_{n}\right) .
$$

Portanto:

$$
\overline{(\exists ! \operatorname{tq} \mathcal{P})}\left(\xi_{1}, \ldots, \xi_{n}\right) \quad \text { se e somente se } \quad(\exists ! \operatorname{tq} \overline{\mathcal{P}})\left(\xi_{1}, \ldots, \xi_{n}\right)
$$

para qualquer $n$-upla $\left(\xi_{1}, \ldots, \xi_{n}\right)$ de objetos virtuais.

(vii) As afirmações seguintes são equivalentes:

$$
\begin{aligned}
& \overline{(\forall \mathcal{P} \mathcal{Q})}\left(\xi_{1}, \ldots, \xi_{n}\right) \\
& \exists m \in \mathbf{N}, \forall i>m,(\forall \mathcal{P} \mathcal{Q})\left(x_{1}^{i}, \ldots, x_{n}^{i}\right) \\
& \exists m \in \mathbf{N}, \forall i>m, \forall\left(y_{1}, \ldots, y_{k}\right) \in \mathcal{U}^{n} \text { tal que } \mathcal{P}\left(y_{1}, \ldots, y_{k}\right), \mathcal{Q}\left(y_{1}, \ldots, y_{k}, x_{1}^{i}, \ldots, x_{n}^{i}\right) .
\end{aligned}
$$


Esta última afirmação equivale a dizer:

Para quaisquer $k$ sequências $\left(y_{l}^{1}, y_{l}^{2}, \ldots\right)$ de objetos de $\mathcal{U}(l=1, \ldots, k)$

tais que $\mathcal{P}\left(y_{1}^{i}, \ldots, y_{k}^{i}\right), \exists m \in \mathbf{N}, \forall i>m, \mathcal{Q}\left(y_{1}^{i}, \ldots, y_{k}^{i}, x_{1}^{i}, \ldots, x_{n}^{i}\right)$.

Fazendo $v_{l}=\left\langle y_{l}^{1}, y_{l}^{2}, \ldots\right\rangle$, para $l=1, \ldots, k$, vemos que isto é o mesmo que afirmar:

$$
\begin{aligned}
& \text { Para toda } k \text {-upla }\left(v_{1}, \ldots, v_{k}\right) \in \overline{\mathcal{U}}^{k} \text { tal que } \mathcal{P}\left(v_{1}, \ldots, v_{k}\right), \\
& \text { temos } \mathcal{Q}\left(v_{1}, \ldots, v_{k}, \xi_{1}, \ldots, \xi_{n}\right)
\end{aligned}
$$

Portanto:

$$
\overline{(\forall \mathcal{P} \mathcal{Q})}\left(\xi_{1}, \ldots, \xi_{n}\right) \quad \text { se e somente se } \quad(\forall \overline{\mathcal{P}} \overline{\mathcal{Q}})\left(\xi_{1}, \ldots, \xi_{n}\right)
$$

para qualquer $n$-upla $\left(\xi_{1}, \ldots, \xi_{n}\right)$ de objetos virtuais.

O item (vii) do lema acima não seria válido se o domínio $\mathcal{P}$ da quantificação universal envolvesse as variáveis correntes do escopo onde esta construção lógica está sendo realizada. Para verificar isto, consideremos a operação que leva dois predicados binários quaisquer $\mathcal{P}$ e $\mathcal{Q}$ no predicado unário $\left(\forall_{*} \mathcal{P} \mathcal{Q}\right)$ dado por:

$$
\begin{gathered}
\left(\forall_{*} \mathcal{P} \mathcal{Q}\right)(x) \quad \text { se e somente se } \\
\forall y \in \mathcal{U} \text { tal que } \mathcal{P}(y, x), \text { temos } \mathcal{Q}(y, x),
\end{gathered}
$$

bem como a operação análoga sobre predicados virtuais.

A fim de obter um contra-exemplo para a afirmação:

$$
\overline{\left(\forall_{*} \mathcal{P Q}\right)}=\left(\forall_{*} \overline{\mathcal{P}} \overline{\mathcal{Q}}\right)
$$

basta considerar um universo constituído por apenas dois elementos distintos, digamos $\mathcal{U}=\{a, b\}$, e tomar os predicados binários:

$$
\mathcal{P}(x, y) \quad \text { se e somente se } \quad x=y=a .
$$

e

$$
\mathcal{Q}(x, y) \quad \text { é falso, para qualquer par }(x, y) \in \mathcal{U}^{2}
$$

É claro que:

$$
\left(\forall_{*} \mathcal{P} \mathcal{Q}\right)(x) \quad \text { se e somente se } \quad x=b,
$$

$\log 0:$

$$
\overline{\left(\forall_{*} \mathcal{P Q}\right)}(\xi) \quad \text { se e somente se } \quad \xi=\bar{b}
$$


Assim, para o objeto virtual:

$$
\gamma=\langle a, b, a, b, a, b, \ldots\rangle \in \overline{\mathcal{U}}
$$

temos que $\overline{\left(\forall_{*} \mathcal{P Q}\right)}(\gamma)$ é falso, pois $\gamma \neq \bar{b}$. Entretanto, $\left(\forall_{*} \overline{\mathcal{P}} \overline{\mathcal{Q}}\right)(\gamma)$ é verdadeiro, pois não existe $\xi \in \overline{\mathcal{U}}$ tal que $\overline{\mathcal{P}}(\xi, \gamma)$.

Esse é o motivo da restrição que fizemos às quantificações universais admissíveis. É fácil de ver que o TEV, na forma como foi enunciado acima, também não seria válido se admitíssemos as operações lógicas de negação ou de disjunção.

Provaremos agora o TEV: se uma afirmação matemática clara sobre $\mathcal{U}$ envolve apenas construções lógicas admissíveis, em número finito, então sua extensão virtual será verdadeira se e somente se ela própria for verdadeira.

Para tanto, consideremos uma afirmação matemática clara sobre $\mathcal{U}$, cujo enunciado envolva apenas construções lógicas admissíveis, em número finito, combinadas com:

(a) os objetos específicos $a_{1}, a_{2}, \ldots, a_{l}$;

(b) os predicados $\mathcal{P}_{1}, \mathcal{P}_{2}, \ldots, \mathcal{P}_{p}$; e

(c) os funcionais $\mathcal{F}_{1}, \mathcal{F}_{2}, \ldots, \mathcal{F}_{f}$.

Esta afirmação é uma conjunção de duas outras afirmações matemáticas sobre $\mathcal{U}$, ou equivale necessariamente a algum dos seguintes enunciados:

(i) uma predicação do tipo:

$$
\mathcal{P}\left(a_{i}\right) \quad i \in\{1, \ldots, l\}
$$

(ii) uma quantificação existencial:

Existe $x \in \mathcal{U}$ tal que $\mathcal{P}(x)$

(iii) uma quantificação exclusiva:

Existe um e um único $x \in \mathcal{U}$ tal que $\mathcal{P}(x)$;

$\mathrm{Ou}$

(iv) uma quantificação universal:

$$
\begin{aligned}
& \text { Para toda } n \text {-upla }\left(x_{1}, \ldots, x_{n}\right) \in \mathcal{U}^{n} \text { tal que } \\
& \mathcal{P}\left(x_{1}, \ldots, x_{n}\right), \text { temos } \mathcal{Q}\left(x_{1}, \ldots, x_{n}\right)
\end{aligned}
$$

onde, em qualquer dos casos, os predicados $\mathcal{P}$ e $\mathcal{Q}$ podem ser obtidos, a partir dos objetos específicos $a_{1}, a_{2}, \ldots, a_{l}$ dos funcionais $\mathcal{S}_{i}^{n}, \mathcal{F}_{1}, \mathcal{F}_{2}, \ldots, \mathcal{F}_{f}$ e dos predica$\operatorname{dos} \mathcal{I}, \mathcal{P}_{1}, \mathcal{P}_{2}, \ldots, \mathcal{P}_{p}$, por um número finito de aplicações sucessivas das operações de 
avaliação parcial, composição funcional, composição predicativa, conjunção, quantificação existencial, quantificação exclusiva, e quantificação universal. O lema anterior mostra que a extensão virtual do resultado de cada uma destas operações é logicamente equivalente à substituição de seus operandos pelas respectivas extensões virtuais. As extensões virtuais do predicado de igualdade $\mathcal{I}$ e dos funcionais seletores $\mathcal{S}_{i}^{n}$ também preservam os respectivos sentidos lógicos (pelos dois primeiros lemas desta seção). Portanto, resta demonstrar apenas a equivalência entre afirmações dos quatro tipos acima e aquelas que obtemos substituindo nelas os predicados $\mathcal{P}$ e $\mathcal{Q}$ por suas respectivas extensões virtuais:

(i) É trivial ver que $\mathcal{P}\left(a_{i}\right)$ se e somente se $\overline{\mathcal{P}}\left(\overline{a_{i}}\right)$;

(ii) A afirmação:

Existe $x \in \mathcal{U}$ tal que $\mathcal{P}(x)$

equivale a

Existe $\xi \in \overline{\mathcal{U}}$ tal que $\overline{\mathcal{P}}(\xi)$,

pois $\overline{\mathcal{P}} \subset \overline{\mathcal{U}}$ é vazio se e somente se $\mathcal{P} \subset \mathcal{U}$ for vazio.

(iii) A afirmação

Existe um e um único $x \in \mathcal{U}$ tal que $\mathcal{P}(x)$

é equivalente a

Existe um e um único $\xi \in \overline{\mathcal{U}}$ tal que $\overline{\mathcal{P}}(\xi)$,

pois $\overline{\mathcal{P}} \subset \overline{\mathcal{U}}$ é unitário se e somente se $\mathcal{P} \subset \mathcal{U}$ for unitário.

(iv) A afirmação:

Para toda $n$-upla $\left(x_{1}, \ldots, x_{n}\right) \in \mathcal{U}^{n}$ tal que $\mathcal{P}\left(x_{1}, \ldots, x_{n}\right)$,

temos $\mathcal{Q}\left(x_{1}, \ldots, x_{n}\right)$

equivale a

Para toda $n$-upla $\left(\xi_{1}, \ldots, \xi_{n}\right) \in \overline{\mathcal{U}}^{n}$ tal que $\overline{\mathcal{P}}\left(\xi_{1}, \ldots, \xi_{n}\right)$,

temos $\overline{\mathcal{Q}}\left(\xi_{1}, \ldots, \xi_{n}\right)$,

pois $\overline{\mathcal{P}} \subset \overline{\mathcal{Q}}$ se e somente se $\mathcal{P} \subset \mathcal{Q}$.

Completamos assim a demonstração do TEV.

\subsection{Semelhança e Dessemelhança}

A terminologia seguinte será usada muitas vezes ao longo deste trabalho:

DEFINIÇÃo: Diremos que um objeto virtual foi transferido de $\mathcal{U}$, ou simplesmente que é um objeto transferido, quando ele for a extensão virtual de algum objeto real. Analogamente, diremos que um predicado virtual é transferido quando ele for a extensão virtual de algum predicado real, e que um funcional virtual é transferido quando ele for a extensão virtual de algum funcional real. 
Vimos na seção anterior que a extensão virtual do predicado binário de igualdade entre objetos de $\mathcal{U}$ é o predicado virtual de igualdade entre objetos virtuais, portanto este é um predicado transferido. Por outro lado, a extensão virtual do predicado binário de desigualdade entre objetos de $\mathcal{U}$ não é o predicado de desigualdade entre objetos de $\overline{\mathcal{U}}$ :

DEFIniÇÃo: Diremos que dois objetos virtuais são dessemelhantes quando eles satisfizerem a extensão virtual do predicado $\mathcal{P} \subset \mathcal{U}^{2}$ definido por:

$$
\mathcal{P}(a, b): \quad a \neq b .
$$

Além disso, diremos que dois objetos virtuais são semelhantes quando eles não forem dessemelhantes. Usaremos a notação $\alpha \asymp \beta$ para indicar que $\alpha$ e $\beta$ são semelhantes, e escreveremos $\beta \nprec \alpha$ quando $\alpha$ e $\beta$ forem dessemelhantes.

Segundo as definições acima, se

$$
\alpha=\left\langle a_{1}, a_{2}, a_{3}, \ldots\right\rangle \quad \text { e } \quad \beta=\left\langle b_{1}, b_{2}, b_{3}, \ldots\right\rangle
$$

então $\alpha$ e $\beta$ são dessemelhantes (isto é: $\alpha \nprec \beta$ ) quando

$$
\exists k \in \mathbf{N}, \forall i>k, a_{i} \neq b_{i}
$$

Portanto, $\alpha$ e $\beta$ são semelhantes (isto é: $\alpha \asymp \beta$ ) quando

$$
\forall k \in \mathbf{N}, \exists i>k, a_{i}=b_{i}
$$

É fácil verificar então que:

Proposição: Para quaisquer objetos virtuais $\alpha$ e $\beta$ :

(i) $\alpha=\beta$ implica $\alpha \asymp \beta$, mas $\alpha \asymp \beta$ não implica $\alpha=\beta$;

(ii) $\alpha \nprec \beta$ implica $\alpha \neq \beta$, mas $\alpha \neq \beta$ não implica $\alpha \nprec \beta$;

(iii) $\alpha \asymp \beta$ se e somente se $\beta \asymp \alpha$; e

(iv) $\alpha \nprec \beta$ se e somente se $\beta \nsim \alpha$.

ProposiçÃo: Para quaisquer objetos virtuais transferidos $\bar{a}$ e $\bar{b}$ :

(i) $\bar{a} \asymp \bar{b}$ se e somente se $\bar{a}=\bar{b}$; e

(ii) $\bar{a} \nprec \bar{b}$ se e somente se $\bar{a} \neq \bar{b}$.

Em outras palavras: quando restritas a objetos transferidos, a semelhança equivale à igualdade, e a dessemelhança equivale à desigualdade. 
É claro que, para todo predicado virtual transferido $\overline{\mathcal{P}} \subset \overline{\mathcal{U}}^{n}$ :

$$
\overline{\mathcal{P}}\left(\overline{a_{1}}, \ldots, \overline{a_{n}}\right) \quad \text { se e somente se } \quad \mathcal{P}\left(a_{1}, \ldots, a_{n}\right)
$$

As proposições acima nos mostram então que a semelhança e a desigualdade entre objetos virtuais são predicados virtuais binários que não foram transferidos de $\overline{\mathcal{U}}$. A dessemelhança e igualdade entre objetos virtuais, ao contrário, são predicados virtuais binários transferidos.

Não é difícil ver também que predicado virtual de semelhança, apesar de ser reflexivo e simétrico, não é transitivo, não sendo portanto uma relação de equivalência em $\overline{\mathcal{U}}$.

\subsection{Inclusão Lógica e Embaralhamento}

A definição seguinte visa apenas simplificar a terminologia:

DEFIniÇÃo: Chamaremos os predicados unários de atributos, e os predicados virtuais unários de atributos virtuais. Além disso, diremos que um atributo virtual foi transferido de $\mathcal{U}$, ou simplesmente que é um atributo transferido, quando ele for a extensão virtual de algum atributo real.

Introduzimos agora o predicado virtual de inclusão lógica:

DEFiniÇÃo: Diremos que um objeto virtual $\alpha \in \overline{\mathcal{U}}$ está dentro de outro objeto virtual $\beta \in \overline{\mathcal{U}}$ quando $\alpha$ satisfizer todo atributo transferido que for satisfeito por $\beta$. Em outras palavras, $\alpha$ está dentro de $\beta$ quando

$$
\text { para todo } \mathcal{P} \subset \mathcal{U}, \quad \overline{\mathcal{P}}(\beta) \quad \text { implica } \quad \overline{\mathcal{P}}(\alpha) \text {. }
$$

Usaremos a notação $\alpha \triangleleft \beta$ para indicar que $\alpha$ está dentro de $\beta$. Equivalentemente, escreveremos $\beta \triangleright \alpha$, e diremos que $\beta$ contém $\alpha$.

Fixando a seguinte notação relativa a sequências $\left(a_{i}\right)$ de objetos reais:

$$
a[k]=\left\{a_{j} \in \mathcal{U} \mid j \geq k\right\}
$$

temos que, se

$$
\alpha=\left\langle a_{1}, a_{2}, a_{3}, \ldots\right\rangle \quad \quad \text { e } \quad \beta=\left\langle b_{1}, b_{2}, b_{3}, \ldots\right\rangle
$$

então $\alpha \triangleleft \beta$ quando

$$
\forall k \in \mathbf{N}, \exists i \in \mathbf{N}, a[i] \subset b[k] .
$$

Assim, não é difícil ver que: 
Proposição: Se $\bar{b} \in \overline{\mathcal{U}}$ é um objeto virtual transferido, e $\alpha \in \overline{\mathcal{U}}$ um objeto virtual qualquer, então:

$$
\alpha \triangleleft \bar{b} \quad \text { implica } \quad \alpha=\bar{b} \text {. }
$$

Portanto, quando restrita a objetos transferidos, a inclusão lógica equivale à igualdade:

$$
\bar{a} \triangleleft \bar{b} \quad \text { se e somente se } \quad \bar{a}=\bar{b} .
$$

Isto nos mostra que a inclusão lógica é um predicado virtual binário que não foi transferido de $\mathcal{U}$. É fácil verificar também que:

ProposiçÃo: Para quaisquer objetos virtuais $\alpha, \beta$ e $\gamma$ :

(i) $\alpha \triangleleft \alpha$;

(ii) $\alpha \triangleleft \beta$ e $\beta \triangleleft \gamma$ implicam $\alpha \triangleleft \gamma$.

A inclusão lógica, apesar de reflexiva e transitiva, não satisfaz a tricotomia, não sendo portanto uma ordem total entre objetos virtuais.

É importante observar que não existe qualquer relação direta entre inclusão lógica e semelhança. Por exemplo, não é verdade que $\alpha \asymp \beta$ implique $\alpha \triangleleft \beta$ ou $\beta \triangleleft \alpha$. Além disso:

$$
\alpha \triangleleft \beta \text { e } \beta \triangleleft \alpha \quad \text { não implicam } \quad \alpha \asymp \beta \text {. }
$$

Como contra-exemplo, basta considerar dois objetos reais distintos $a$ e $b$, e tomar

$$
\alpha=\langle a, b, a, b, a, b, \ldots\rangle \quad \text { e } \quad \beta=\langle b, a, b, a, b, a, \ldots\rangle
$$

Podemos assim introduzir outro predicado virtual binário reflexivo, distinto da igualdade e da semelhança:

DefiniÇÃo: Diremos que dois objetos virtuais $\alpha$ e $\beta$ estão embaralhados quando tivermos, simultaneamente, $\alpha \triangleleft \beta$ e $\beta \triangleleft \alpha$. Neste caso, escreveremos $\alpha \bowtie \beta$.

Esse predicado virtual de embaralhamento é, claramente, uma relação de equivalência entre objetos virtuais.

Utilizando a caracterização acima do predicado virtual de inclusão em termos de sequências representantes, não é difícil verificar também que:

Proposição: Se $\overline{\mathcal{F}}$ é um funcional unário transferido que pode ser calculado em um objeto virtual $\beta$, e $\alpha \triangleleft \beta$, então $\overline{\mathcal{F}}$ pode ser calculado em $\alpha$ e $\overline{\mathcal{F}}(\alpha) \triangleleft \overline{\mathcal{F}}(\beta)$. 


\subsection{Mistura Lógica}

Introduzimos a seguir o conceito de mistura lógica de objetos virtuais:

DefiniÇÃo: Seja $\mathcal{P} \subset \mathcal{U}^{3}$ o predicado ternário dado por:

$$
\mathcal{P}(a, b, c): \quad a=b \quad \text { ou } \quad a=c .
$$

Diremos que um objeto virtual $\alpha$ é uma mistura de dois outros objetos virtuais $\beta$ e $\gamma$ quando eles satisfizerem a extensão virtual $\overline{\mathcal{P}}$ do predicado definido acima, isto é, quando $\overline{\mathcal{P}}(\alpha, \beta, \gamma)$.

Segundo essa definição, se

$$
\alpha=\left\langle a_{i}\right\rangle, \quad \beta=\left\langle b_{i}\right\rangle \quad \text { e } \quad \gamma=\left\langle c_{i}\right\rangle,
$$

então $\alpha$ é uma mistura de $\beta$ e $\gamma$ quando

$$
\exists k \in \mathbf{N}, \forall i>k, \quad a_{i}=b_{i} \text { ou } a_{i}=c_{i} .
$$

É claro que $\alpha$ é uma mistura de $\beta$ e $\gamma$ se e somente se $\alpha$ for uma mistura de $\gamma$ e $\beta$. Não é difícil ver também que $\alpha$ ser uma mistura de $\beta$ e $\gamma$ não implica $\alpha=\beta$ ou $\alpha=\gamma$, mas temos que:

Proposição: Se $\alpha$ é uma mistura de $\beta$ e $\gamma$, e $\alpha \nprec \beta$, então $\alpha=\gamma$.

Prova: Seja $\alpha=\left\langle a_{i}\right\rangle, \beta=\left\langle b_{i}\right\rangle$ e $\gamma=\left\langle c_{i}\right\rangle$. Como $\alpha$ é uma mistura de $\beta$ e $\gamma$, temos que:

$$
\exists k_{1} \in \mathbf{N}, \forall i>k_{1}, \quad a_{i}=b_{i} \text { ou } a_{i}=c_{i} .
$$

Por outro lado:

$$
\exists k_{2} \in \mathbf{N}, \forall i>k_{2}, a_{i} \neq b_{i},
$$

pois $\alpha \nprec \beta$. Fazendo então $k=\operatorname{máx}\left\{k_{1}, k_{2}\right\}$ concluímos que:

$$
\exists k \in \mathbf{N}, \forall i>k, a_{i}=c_{i},
$$

ou seja, $\alpha=\gamma$.

Entretanto, esse resultado não permanece válido se substituirmos a hipótese $\alpha \nsim \beta$ por $\alpha \neq \beta$. Como contra-exemplo, basta tomar dois objetos reais distintos $b$ e $c$, e considerar:

$$
\alpha=\langle b, c, b, c, b, c, \ldots\rangle, \quad \beta=\bar{b} \quad \text { e } \quad \gamma=\bar{c} .
$$

A relevância do conceito de mistura lógica é devida, em grande parte, aos resultados seguintes: 
Proposição: Se $\alpha$ é uma mistura de $\beta$ e $\gamma$, e $\overline{\mathcal{P}}$ um atributo virtual transferido tal que $\overline{\mathcal{P}}(\beta)$ e $\overline{\mathcal{P}}(\gamma)$, então $\overline{\mathcal{P}}(\alpha)$.

Prova: Seja $\alpha=\left\langle a_{i}\right\rangle, \beta=\left\langle b_{i}\right\rangle$ e $\gamma=\left\langle c_{i}\right\rangle$. Pelas hipóteses:

$$
\begin{gathered}
\exists k_{1} \in \mathbf{N}, \forall i>k_{1}, \quad a_{i}=b_{i} \text { ou } a_{i}=c_{i}, \\
\exists k_{2} \in \mathbf{N}, \forall i>k_{2}, \mathcal{P}\left(b_{i}\right),
\end{gathered}
$$

$\mathrm{e}$

$$
\exists k_{3} \in \mathbf{N}, \forall i>k_{3}, \mathcal{P}\left(c_{i}\right) .
$$

Tomando $k=\operatorname{máx}\left\{k_{1}, k_{2}, k_{3}\right\}$ concluímos que

$$
\exists k \in \mathbf{N}, \forall i>k, \mathcal{P}\left(a_{i}\right) .
$$

Ou seja, $\overline{\mathcal{P}}(\alpha)$.

Proposição: Se $\overline{\mathcal{F}}$ é um funcional unário transferido que pode ser calculado em dois objetos virtuais $\beta$ e $\gamma$, e $\alpha$ é uma mistura de $\beta$ e $\gamma$, então $\overline{\mathcal{F}}$ pode ser calculado em $\alpha$ e, além disso, $\overline{\mathcal{F}}(\alpha)$ é um mistura de $\overline{\mathcal{F}}(\beta)$ e $\overline{\mathcal{F}}(\gamma)$.

Prova: Seja $\alpha=\left\langle a_{i}\right\rangle, \beta=\left\langle b_{i}\right\rangle, \gamma=\left\langle c_{i}\right\rangle, \overline{\mathcal{F}}(\beta)=\left\langle x_{i}\right\rangle$ e $\overline{\mathcal{F}}(\gamma)=\left\langle y_{i}\right\rangle$. Pelas hipóteses:

$$
\begin{gathered}
\exists k_{1} \in \mathbf{N}, \forall i>k_{1}, \quad a_{i}=b_{i} \text { ou } a_{i}=c_{i}, \\
\exists k_{2} \in \mathbf{N}, \forall i>k_{2}, \mathcal{F}\left(b_{i}\right)=x_{i},
\end{gathered}
$$

e

$$
\exists k_{3} \in \mathbf{N}, \forall i>k_{3}, \mathcal{F}\left(c_{i}\right)=y_{i}
$$

Tomando $k=\operatorname{máx}\left\{k_{1}, k_{2}, k_{3}\right\}$ concluímos que $\overline{\mathcal{F}}$ pode ser calculado em $\alpha$ e, além disso:

$$
\exists k \in \mathbf{N}, \forall i>k, \quad \mathcal{F}\left(a_{i}\right)=x_{i} \text { ou } \mathcal{F}\left(a_{i}\right)=y_{i}
$$

Ou seja, $\overline{\mathcal{F}}(\alpha)$ é um mistura de $\overline{\mathcal{F}}(\beta)$ e $\overline{\mathcal{F}}(\gamma)$.

\subsection{Decomposição Lógica}

O TEV não se aplica a uma afirmação matemática que contenha uma disjunção, isto é, uma construção lógica correspondente à seguinte operação com predicados:

DefiniçÃo: Seja $\mathcal{P}$ e $\mathcal{Q}$ dois predicados $n$-ários quaisquer. Chamaremos de disjunção de $\mathcal{P}$ e $\mathcal{Q}$ o predicado $n$-ário $\mathcal{D}$ dado por

$$
\mathcal{D}\left(x_{1}, \ldots, x_{n}\right): \quad \mathcal{P}\left(x_{1}, \ldots, x_{n}\right) \text { ou } \mathcal{Q}\left(x_{1}, \ldots, x_{n}\right) .
$$


Ou seja, a disjunção é a operação lógica correspondente à operação conjuntista de união: a disjunção de $\mathcal{P} \subset \mathcal{U}^{n}$ e $\mathcal{Q} \subset \mathcal{U}^{n}$ é o conjunto $\mathcal{D}=\mathcal{P} \cup \mathcal{Q}$.

Vamos demonstrar nesta seção alguns resultados a respeito da extensão virtual de disjunções. Temos inicialmente:

Lema DA NegaÇÃo: Seja $\mathcal{D} \subset \mathcal{U}$ a disjunção de dois atributos $\mathcal{P}$ e $\mathcal{Q}$ quaisquer. Se $\alpha$ é um objeto virtual que satisfaz $\overline{\mathcal{D}}$, mas não satisfaz $\overline{\mathcal{Q}}$, então existe $\beta \triangleleft \alpha$ tal que $\overline{\mathcal{P}}(\beta)$. Prova: Seja $\alpha=\left\langle a_{1}, a_{2}, a_{3}, \ldots\right\rangle$. A partir da sequência $\left(a_{i}\right)$ construímos uma outra, $\left(b_{i}\right)$, do seguinte modo: para qualquer $i \in \mathbf{N}$, existe $j \geq i$ tal que $a_{j}$ não satisfaz $\mathcal{Q}$, pois $\alpha$ não satisfaz $\overline{\mathcal{Q}}$. Assim, para cada $i \in \mathbf{N}$, definimos $b_{i}=a_{j}$, onde $j$ é o menor natural tal que $j \geq i$ e $a_{j}$ não satisfaz $\mathcal{Q}$. O objeto virtual $\beta=\left\langle b_{1}, b_{2}, b_{3}, \ldots\right\rangle \in \overline{\mathcal{U}}$ tem as duas propriedades requeridas.

Esse resultado, apesar de ser útil, não é suficiente para tratarmos a extensão de algumas disjunções de atributos. A decomposição lógica que introduzimos a seguir envolve, simultaneamente, as noções de semelhança, inclusão e mistura:

DefiniçÃo: Diremos que um objeto virtual $\alpha$ é decomposto por dois outros objetos virtuais $\beta$ e $\gamma$, ou, equivalentemente, que $\beta$ e $\gamma$ decompõem $\alpha$, quando tivermos:

(i) $\beta \triangleleft \alpha \quad$ e $\quad \gamma \triangleleft \alpha$;

(ii) $\beta \nprec \gamma$; e

(iii) $\alpha$ é uma mistura de $\beta$ e $\gamma$.

LEMA DA DisJunÇão: Seja $\mathcal{D} \subset \mathcal{U}$ a disjunção de dois atributos $\mathcal{P}$ e $\mathcal{Q}$ quaisquer. Se $\alpha$ é um objeto virtual que satisfaz $\overline{\mathcal{D}}$, mas não satisfaz $\overline{\mathcal{P}}$ nem $\overline{\mathcal{Q}}$, então existe uma decomposição de $\alpha$ em dois objetos virtuais $\beta$ e $\gamma$ tais que $\overline{\mathcal{P}}(\beta)$ e $\overline{\mathcal{Q}}(\gamma)$.

Prova: Seja $\alpha=\left\langle a_{1}, a_{2}, a_{3}, \ldots\right\rangle$. A partir da sequência $\left(a_{i}\right)$ vamos construir duas outras, que representaremos por $\left(b_{i}\right)$ e $\left(c_{i}\right)$. Inicialmente, chamemos de distinguidos os naturais $j$ que têm a seguinte propriedade:

$$
\mathcal{P}\left(a_{j}\right), \text { mas não } \mathcal{Q}\left(a_{j}\right) \text {. }
$$

Como $\alpha$ satisfaz $\overline{\mathcal{D}}$, mas não satisfaz $\overline{\mathcal{P}}$, existe uma quantidade infinita de naturais distinguidos. Por outro lado, como $\alpha$ não satisfaz $\overline{\mathcal{Q}}$, concluímos que também existe uma quantidade infinita de naturais não-distinguidos.

Para cada $i \in \mathbf{N}$, definimos:

(i) $b_{i}=a_{j}$, onde $j$ é o menor natural não distinguido com $j \geq i$; e

(ii) $c_{i}=a_{j}$, onde $j$ é o menor natural distinguido com $j \geq i$.

Basta agora fazer $\beta=\left\langle b_{i}\right\rangle$ e $\gamma=\left\langle c_{i}\right\rangle$ e verificar que estes dois objetos virtuais têm todas as propriedades requeridas. 
Finalizamos este capítulo com um corolário útil do resultado acima:

LEMA DA SEMElHanÇA: Seja $\alpha$ um objeto virtual qualquer, e $\bar{b}$ um objeto virtual transferido. Se $\alpha$ e $\bar{b}$ são semelhantes, porém distintos, então existe um objeto virtual $\gamma$ tal que $\bar{b}$ e $\gamma$ decompõem $\alpha$. 


\section{Capítulo 2}

\section{Cálculo de Funções Reais}

Mostraremos agora como o formalismo introduzido no capítulo anterior pode ser utilizado para organizar as idéias básicas do Cálculo Infinitesimal. Não pretendemos desenvolver aqui um tratado completo sobre o assunto, mas apenas apresentar um esboço geral desta organização alternativa, que deixe claro que ela pode ser completada. Esperamos também fornecer elementos suficientes para permitir uma comparação prévia entre a formulação que estamos propondo e a weierstrasiana, baseada exclusivamente no conceito de limite. Estes dois formalismos não são incompatíveis: nada impede que eles sejam sobrepostos, isto é, aplicados simultaneamente em uma mesma exposição. Entretanto, para maior clareza, optamos por mencionar o mínimo possível os conceitos tradicionais, deixando para uma outra oportunidade o estudo da utilidade ou conveniência desta sobreposição.

\subsection{Números Virtuais}

Até aqui, não fizemos nenhuma hipótese sobre a natureza dos objetos pertencentes ao universo de discurso matemático $\mathcal{U}$ fixado no início do processo de extensão virtual. Suponhamos agora que os números reais sejam todos objetos deste universo:

DefiniÇÃo: Seja $\mathcal{P} \subset \mathcal{U}$ o predicado unário:

$$
\mathcal{P}(a): \quad a \text { é um número real. }
$$

A extensão virtual deste predicado define um tipo de objeto virtual que chamaremos de número virtual:

$$
\overline{\mathcal{P}}(\alpha): \quad \alpha \text { é um número virtual. }
$$

Assim, um objeto virtual

$$
\alpha=\left\langle a_{1}, a_{2}, a_{3}, \ldots\right\rangle \in \overline{\mathcal{U}}
$$


é um número virtual, ou simplesmente um virtual, se, a partir de um certo valor do índice, todos os $a_{i}$ forem números reais.

Por exemplo, a classe de equivalência da sequência $(1,2,3, \ldots)$ é um número virtual, que representaremos por $\infty$. Ou seja:

$$
\infty=\langle 1,2,3, \ldots\rangle \in \overline{\mathcal{U}}
$$

Usaremos a palavra da língua portuguesa "infinito", como substantivo, para designar este objeto virtual, isto é, para ler o símbolo $\infty$. Como adjetivo, ela manterá seu significado usual: "não-finito".

Denotaremos o conjunto de todos os números reais por $\mathbf{R}$, e o conjunto de todos os números virtuais por $\overline{\mathbf{R}}$. Como estamos considerando os números reais como objetos elementares, não há problema em identificá-los como as respectivas classes de sequências constantes:

$$
a=\bar{a}=\langle a, a, a, \ldots\rangle, \quad \forall a \in \mathbf{R} .
$$

Estamos considerando, portanto, $\mathbf{R}$ como subconjunto de $\overline{\mathbf{R}}$.

Cada subconjunto $\mathrm{A} \subset \mathbf{R}$ pode ser considerado como um predicado unário, cuja extensão virtual é um subconjunto $\overline{\mathrm{A}}$ de $\overline{\mathbf{R}}$.

Temos, por exemplo, o subconjunto $\mathbf{N} \subset \mathbf{R}$ dos números naturais (inteiros positivos), correspondente ao predicado unário "ser um número natural", que pode ser estendido aos números virtuais, definindo um subconjunto $\overline{\mathbf{N}}$ de $\overline{\mathbf{R}}$. Um número virtual $\alpha=$ $\left\langle a_{1}, a_{2}, a_{3}, \ldots\right\rangle$ é um natural virtual, isto é, $\alpha \in \overline{\mathbf{N}}$, quando todos os $a_{i}$ são números naturais, a partir de um certo valor do índice. É fácil ver que:

$$
\infty \in \overline{\mathbf{N}}, \quad \operatorname{mas}-1 \notin \overline{\mathbf{N}} \text {. }
$$

A relação de ordem < entre números reais é um predicado binário:

$$
\mathcal{P}(a, b): \quad a<b
$$

Temos portanto sua extensão virtual:

$$
\overline{\mathcal{P}}(\alpha, \beta): \quad \alpha \overline{<} \beta
$$

Segundo esta definição, se

$$
\alpha=\left\langle a_{1}, a_{2}, a_{3}, \ldots\right\rangle \quad \text { e } \quad \beta=\left\langle b_{1}, b_{2}, b_{3}, \ldots\right\rangle
$$

então $\alpha \overline{<} \beta$ se e somente se existir $k \in \mathbf{N}$ tal que, para todo $i>k$, tivermos $a_{i}<b_{i}$. 
É fácil ver, por exemplo, que:

$$
\forall a \in \mathbf{R}, \quad a \overline{<} \infty
$$

A fim de não sobrecarregar a notação, omitiremos a barra na desigualdade acima, escrevendo simplesmente:

$$
\forall a \in \mathbf{R}, \quad a<\infty .
$$

Não há risco de confusão, pois o símbolo $\infty$ indica claramente que se trata da extensão virtual de $<$.

Podemos também considerar a ordem entre números reais na forma do predicado binário:

$$
\mathcal{P}(a, b): \quad a \leq b,
$$

cuja extensão virtual é:

$$
\overline{\mathcal{P}}(\alpha, \beta): \quad \alpha \overline{\leq} \beta
$$

Também é fácil verificar que:

$$
\forall a \in \mathbf{R}, \quad a \leq \infty
$$

e podemos omitir a barra também nesta desigualdade, escrevendo apenas:

$$
\forall a \in \mathbf{R}, \quad a \leq \infty
$$

Omissões das barras que indicam extensões virtuais, como as duas exemplificadas acima, simplificam bastante a notação, e não acarretam ambiguidades se forem feitas criteriosamente. Mas devemos proceder com cuidado, como mostra o exemplo seguinte.

Entre números reais, é claro que:

$$
\text { para quaisquer } a \text { e } b \text { tais que } a \leq b \text {, temos } a<b \text { ou } a=b \text {. }
$$

Entretanto, a afirmação análoga:

$$
\text { para quaisquer } \alpha \text { e } \beta \text { tais que } \alpha \leq \beta \text {, temos } \alpha \overline{<} \beta \text { ou } \alpha=\beta \text {, }
$$

não vale para números virtuais. Contra-exemplo: se $\alpha=\langle 1,2,1,2,1,2, \ldots\rangle$ então:

$$
\text { é verdade que } \quad \alpha \bar{\Sigma} 2,
$$

mas

$$
\alpha \overline{<} 2 \quad \text { e } \quad \alpha=2 \quad \text { são ambas falsas. }
$$


O TEV não se aplica ao enunciado acima pois ele contém uma disjunção, que não é uma construção lógica admissível. Isto nos mostra que se quisermos usar a notação $\alpha \leq \beta$ para abreviar:

$$
\alpha<\beta \quad \text { ou } \quad \alpha=\beta,
$$

então não poderemos omitir a barra que distingue $\leq$ de sua extensão virtual.

Podemos, é claro, adotar, em cada caso, a convenção que for mais conveniente. Mas devemos sempre fazê-lo de forma clara e explícita, a fim de evitar confusão. Adotaremos, neste trabalho, a prática de representar $\alpha \leq \beta$ simplesmente por $\alpha \leq \beta$. Temos portanto, pelo TEV, que:

$$
\text { se } \quad \alpha<\beta \quad \text { então } \quad \alpha \leq \beta \text {, }
$$

e que:

$$
\text { se } \quad \alpha=\beta \quad \text { então } \quad \alpha \leq \beta .
$$

Mas não é verdade que:

$$
\text { se } \quad \alpha \leq \beta \quad \text { então } \quad \alpha<\beta \quad \text { ou } \quad \alpha=\beta \text {. }
$$

Aplicando o TEV, é fácil concluir que a relação de ordem $\leq$ entre os números virtuais é reflexiva:

$$
\alpha \leq \alpha, \quad \text { para todo } \alpha \in \overline{\mathbf{R}},
$$

antissimétrica:

$$
\alpha \leq \beta \quad \text { e } \quad \beta \leq \alpha \quad \text { implicam } \quad \alpha=\beta
$$

e transitiva:

$$
\alpha \leq \beta \quad \text { e } \quad \beta \leq \gamma \quad \text { implicam } \quad \alpha \leq \gamma .
$$

Mas ela não satisfaz a tricotomia, isto é, não vale que:

$$
\text { para quaisquer } \alpha \text { e } \beta \text {, temos } \alpha \leq \beta \text { ou } \beta \leq \alpha \text {, }
$$

A relação $\leq$ não é, portanto, uma ordem total em $\overline{\mathbf{R}}$. Contra-exemplo: se $\alpha=$ $\langle 0,2,0,2,0,2, \ldots\rangle$ então

$$
\alpha \leq 1 \quad \text { e } \quad 1 \leq \alpha \quad \text { são ambas falsas. }
$$

Assim, vemos que:

$$
\alpha \nless x \quad \text { não implica } \quad \alpha \geq x .
$$

Entretanto:

Proposição: Se $\alpha \nless x$ então existe $\beta \triangleleft \alpha \operatorname{com} \beta \geq x$. 
Prova: Basta aplicar o Lema da Negação aos atributos:

$$
\mathcal{P}(a): \quad a \geq x
$$

e

$$
\mathcal{Q}(a): \quad a<x
$$

Analogamente:

Proposição: Se $\alpha \not \leq x$ então existe $\beta \triangleleft \alpha \operatorname{com} \beta>x$.

A operação de adição de dois números reais pode ser considerada como um funcional binário:

$$
\mathcal{F}(a, b)=a+b
$$

A extensão virtual deste funcional define a soma dois números virtuais:

$$
\overline{\mathcal{F}}(\alpha, \beta)=\alpha \mp \beta
$$

Segundo esta definição, se

$$
\alpha=\left\langle a_{1}, a_{2}, a_{3}, \ldots\right\rangle \quad \text { e } \quad \beta=\left\langle b_{1}, b_{2}, b_{3}, \ldots\right\rangle
$$

então

$$
\alpha \mp \beta=\left\langle\left(a_{1}+b_{1}\right),\left(a_{2}+b_{2}\right),\left(a_{3}+b_{3}\right), \ldots\right\rangle,
$$

onde as somas $\left(a_{i}+b_{i}\right)$ podem ser calculadas a partir de um certo valor do índice $i$.

Temos assim, por exemplo, os números virtuais:

$$
\infty+1=\langle 1+1,2+1,3+1,4+1, \ldots\rangle=\langle 2,3,4,5, \ldots\rangle \in \overline{\mathbf{R}}
$$

$\mathrm{e}$

$$
\infty+\infty=\langle 1+1,2+2,3+3,4+4, \ldots\rangle=\langle 2,4,6,8, \ldots\rangle \in \overline{\mathbf{R}},
$$

onde a "barra" que distingue + de sua extensão virtual foi omitida porque o símbolo $\infty$ é suficiente para indicar que se trata de uma operação virtual.

A operação de multiplicação também pode ser estendida, analogamente, aos números virtuais. Deste modo podemos definir, por exemplo, o produto:

$$
2 . \infty=\langle 2.1,2.2,2.3,2.4, \ldots\rangle=\langle 2,4,6,8, \ldots\rangle \in \overline{\mathbf{R}}
$$

onde a "barra" que deveria distinguir o sinal de multiplicação de sua extensão virtual foi omitida pelo mesmo motivo acima. 
Comparando os dois últimos exemplos de números virtuais, vemos que:

$$
\infty+\infty=2 . \infty
$$

Isso é apenas uma caso particular do fato:

$$
\forall \alpha \in \overline{\mathbf{R}}, \quad \alpha+\alpha=2 . \alpha,
$$

que decorre da extensão, pelo TEV, do enunciado trivial:

$$
\forall a \in \mathbf{R}, \quad a+a=2 . a .
$$

Tendo estendido as operações aritméticas básicas aos números virtuais, cabe verificar quais de suas propriedades permanecem válidas. É fácil ver, aplicando o TEV, que a soma e a multiplicação virtuais são associativas, comutativas, e satisfazem a lei distributiva. Também decorre do TEV que elas têm por elemento neutro, respectivamente, o zero e a unidade (estamos identificando $0=\overline{0}$ e $1=\overline{1}$ ), e que todo número virtual $\alpha$ tem um oposto, que identificaremos por $-\alpha$.

Por outro lado, o enunciado que afirma a existência de inversos:

$$
\forall a \in \mathbf{R} \text { com } \alpha \neq 0, \exists b \in \mathbf{R} \text { tal que } a . b=1
$$

tem por extensão virtual:

$$
\forall \alpha \in \overline{\mathbf{R}} \operatorname{com} \alpha \nprec 0, \exists \beta \in \overline{\mathbf{R}} \text { tal que } \alpha \cdot \beta=1 .
$$

Assim, o TEV não garante que todo virtual diferente de zero seja inversível. Decorre do TEV apenas a inversibilidade dos virtuais dessemelhantes a zero. De fato, existem virtuais não-nulos que não têm inverso, como, por exemplo $\alpha=\langle 0,1,0,1,0,1, \ldots\rangle$, o que nos mostra que $\overline{\mathbf{R}}$ não é um corpo.

Sabemos, pelo TEV, que se $\alpha>0$ ou $\alpha<0$ então $\alpha \nprec 0$, e portanto todo número virtual estritamente positivo ou estritamente negativo é inversivel.

Representaremos o inverso multiplicativo de um virtual $\alpha \nprec 0$ por $\alpha^{-1}$ ou $1 / \alpha$. Por exemplo, o inverso de $\infty$ será representado por $\infty^{-1}$ ou $1 / \infty$ (sabemos que $\infty$ é inversível pois $\infty>0)$.

Além disso, todas as notações e convenções sintáticas universalmente aceitas para as operações algébricas com números reais serão mantidas para os números virtuais. Escreveremos, por exemplo:

$$
\frac{\infty+1}{\infty}=\frac{1}{\infty}(\infty+1)=\frac{\infty}{\infty}+\frac{1}{\infty}=1+\frac{1}{\infty}
$$

$\mathrm{e}$

$$
(\infty+1)^{2}=(\infty+1)(\infty+1)=\infty^{2}+2 \infty+1,
$$

onde o ponto que indica multiplicação foi sistematicamente omitido, como é habitual. 


\subsection{Finitude}

Vamos introduzir agora uma série de noções relativas à finitude dos números virtuais. Antes, porém, precisamos estender a noção de valor absoluto para os números virtuais. Para tanto, basta considerar o funcional:

$$
\mathcal{F}(a)=|a|,
$$

e tomar sua extensão virtual:

$$
\overline{\mathcal{F}}(\alpha)=|\alpha|
$$

Assim,

$$
\left|\left\langle a_{1}, a_{2}, a_{3}, \ldots\right\rangle\right|=\left\langle\left|a_{1}\right|,\left|a_{2}\right|,\left|a_{3}\right|, \ldots\right\rangle,
$$

onde o valores absolutos podem ser tomados a partir de um certo valor do índice.

DefiniÇÃo: Diremos que um número virtual é um infinitésimo, ou que ele é infinitesimal, quando o seu valor absoluto for menor que qualquer real positivo. Em outras palavras, $\varepsilon \in \overline{\mathbf{R}}$ é infinitesimal se para qualquer número real $x>0$ tivermos $|\varepsilon|<x$.

É fácil ver que um número virtual:

$$
\varepsilon=\left\langle e_{1}, e_{2}, e_{3}, \ldots\right\rangle \in \overline{\mathbf{R}}
$$

é infinitesimal quando a sequência representante $\left(e_{i}\right)$ tende a zero. (Essa sequência assume, a partir de um certo valor do índice, apenas valores em $\mathbf{R}$.)

DEFINIÇÃo: Diremos que um número virtual é limitado quando seu valor absoluto for menor que algum número real, isto é:

$$
\lambda \in \overline{\mathbf{R}} \text { é limitado quando existe } x \in \mathbf{R} \text { tal que }|\lambda|<x .
$$

Com as definições acima, todo número infinitesimal é limitado, embora a recíproca não seja verdadeira, como mostra o seguinte fato:

ProposiçÃo: O único número real infinitesimal é o zero, mas todo número real é limitado.

Prova: Para todo número real não nulo $x$, vale que:

$$
|x|>\frac{|x|}{2},
$$

portanto $x \neq 0$ não é infinitesimal.

Por outro lado, para qualquer real $y$, temos que:

$$
|y|<|y|+1,
$$


logo $y$ é limitado.

DefiniçÃo: Diremos que um número virtual é ilimitado quando ele não for limitado, isto é:

$$
\omega \in \overline{\mathbf{R}} \text { é ilimitado quando não existe } x \in \mathbf{R} \text { tal que }|\omega|<x \text {. }
$$

Além disso, diremos que um número virtual é maior que $\mathbf{R}$ quando ele for maior que todos os números reais, ou seja:

$$
\omega>\mathbf{R} \text { quando } \omega>x \text {, para todo } x \in \mathbf{R} \text {. }
$$

Analogamente:

$$
\omega<\mathbf{R} \text { quando } \omega<x \text {, para todo } x \in \mathbf{R} .
$$

Segundo essas definições, é claro que se $\omega>\mathbf{R}$ ou $\omega<\mathbf{R}$ então $\omega$ é ilimitado. Entretanto, a recíproca dessa afirmação não é verdadeira: existem virtuais ilimitados que não são maiores nem menores que $\mathbf{R}$, como, por exemplo:

$$
\langle 0,1,0,2,0,3,0,4, \ldots\rangle \quad \text { ou } \quad\langle-1,2,-3,4,-5,6,-7,8, \ldots\rangle \text {. }
$$

É fácil ver que $\infty>\mathbf{R}$, e portanto $\infty$ é ilimitado. Mas este virtual não é o único maior que $\mathbf{R}:(\infty+1)$ e $2 \infty$, por exemplo, são ambos maiores que $\mathbf{R}$.

Apresentamos abaixo algumas relações básicas entre finitude e as operações de adição e multiplicação:

ProposiçÃo: A soma de dois infinitésimos é também infinitesimal.

Prova: Temos que $|a+b| \leq|a|+|b|$, para quaisquer $a, b \in \mathbf{R}$. O TEV nos garante então que $|\varepsilon+\delta| \leq|\varepsilon|+|\delta|$, para quaisquer virtuais $\varepsilon$ e $\delta$. Se $\varepsilon$ e $\delta$ são infinitésimos então $|\varepsilon|<x / 2$ e $|\delta|<x / 2$, para qualquer real $x>0$. Logo $|\varepsilon+\delta|$ é menor que qualquer real positivo $x$.

ProposiçÃo: O produto de um infinitésimo por um virtual limitado é infinitesimal.

Prova: Pelo TEV, temos que $|\varepsilon \lambda|=|\varepsilon||\lambda|$, para quaisquer virtuais $\varepsilon$ e $\lambda$. Se $\lambda$ é limitado então existe um real positivo $y$ tal que $|\lambda|<y$. Se $\varepsilon$ é infinitesimal então $|\varepsilon|<x / y$, para qualquer $x>0$. Portanto $|\varepsilon \lambda|$ é menor que qualquer real positivo $x$.

Esse resultado nos mostra que o inverso de um infinitésimo, se existir, será necessariamente ilimitado (caso contrário concluiríamos que $1 \in \mathbf{R}$ é infinitesimal). Entretanto, um virtual ilimitado pode não ser inversível, ou ter um inverso que não é infinitesimal. Exemplos: os virtuais $\langle 0,1,0,2,0,3,0,4, \ldots\rangle$ e $\langle 1,2,1,3,1,4,1,5, \ldots\rangle$ são ambos ilimitados, mas o primeiro não é inversível e o segundo tem um inverso não infinitesimal. O que podemos afirmar com certeza é que: 
ProposiÇÃo: Se $\omega>\mathbf{R}$ ou $\omega<\mathbf{R}$ então $\omega$ é inversível, e seu inverso é infinitesimal.

Prova: Se $\omega>\mathbf{R}$ então $\omega>0$, e portanto $\omega$ é inversível. Além disso, temos que $\omega>(1 / x)$, para qualquer $x$ positivo, logo $0<(1 / \omega)<x$, para qualquer real positivo $x$. O caso $\omega<\mathbf{R}$ é análogo.

Proposição: A soma e o produto de dois virtuais limitados são também limitados.

Prova: Se $\lambda$ e $\mu$ são limitados então existem $x$ e $y$ reais tais que $|\lambda|<x$ e $|\mu|<y$. Temos então que:

$$
|\lambda+\mu|<x+y \quad \text { e } \quad|\lambda \mu|<x y
$$

sendo portanto $\lambda+\mu$ e $\lambda \mu$ limitados.

Como consequência, temos que a soma de um virtual ilimitado $\omega$ com um limitado $\lambda$ será necessariamente ilimitada, pois se $\omega+\lambda$ fosse limitada então $\omega=(\omega+\lambda)-\lambda$ também seria.

ProposiÇÃo: Se $\alpha \triangleleft \beta$ então:

(i) $\beta$ infinitesimal implica $\alpha$ infinitesimal;

(ii) $\beta$ limitado implica $\alpha$ limitado;

(iii) $\beta>\mathbf{R}$ implica $\alpha>\mathbf{R}$.

Prova: Para cada $x>0$, seja $\mathcal{P}_{x} \subset \mathcal{U}$ o atributo de números reais definido por:

$$
\mathcal{P}_{x}(a): \quad|a|<x
$$

(i) Temos $\overline{\mathcal{P}_{x}}(\beta)$, para todo $x>0$, uma vez que $\beta$ é infinitesimal. Como $\overline{\mathcal{P}_{x}}$ é um atributo virtual transferido, e $\alpha \triangleleft \beta$, concluímos que:

$$
\overline{\mathcal{P}_{x}}(\alpha), \quad \text { para todo } x>0
$$

Ou seja, $\alpha$ também é infinitesimal.

(ii) Neste caso, existe um $x>0$ tal que $\overline{\mathcal{P}_{x}}(\beta)$. Como $\overline{\mathcal{P}_{x}}$ é um atributo virtual transferido, e $\alpha \triangleleft \beta$, temos que:

$$
\text { existe } x>0 \operatorname{com} \overline{\mathcal{P}_{x}}(\alpha) \text {, }
$$

logo $\alpha$ também é limitado.

(iii) Consideremos agora, para cada $x \in \mathbf{R}$, o atributo de números reais $\mathcal{Q}_{x} \subset \mathcal{U}$ dado por:

$$
\mathcal{Q}_{x}(a): \quad a>x .
$$


Temos então $\overline{\mathcal{Q}_{x}}(\beta)$, para todo $x \in \mathbf{R}$. Como $\overline{\mathcal{Q}_{x}}$ é um atributo virtual transferido, e $\alpha \triangleleft \beta$, concluímos que:

$$
\overline{\mathcal{Q}_{x}}(\alpha), \quad \text { para todo } x \in \mathbf{R}
$$

Logo $\alpha>R$.

Entretanto, $\alpha \triangleleft \beta$, com $\beta$ ilimitado, não implica $\alpha$ ilimitado, como mostra o contraexemplo:

$$
0 \triangleleft\langle 0,1,0,2,0,3,0,4, \ldots\rangle .
$$

Usando os atributos transferidos $\overline{\mathcal{P}_{x}}$ e $\overline{\mathcal{Q}_{x}}$ da demonstração anterior, não é difícil verificar também que:

Proposição: Se $\alpha$ é uma mistura de $\beta$ e $\gamma$, então:

(i) $\beta$ e $\gamma$ infinitesimais implica $\alpha$ infinitesimal;

(ii) $\beta$ e $\gamma$ limitados implica $\alpha$ limitado; e

(iii) $\beta>\mathbf{R}$ e $\gamma>\mathbf{R}$ implica $\alpha>\mathbf{R}$.

\subsection{Proximidade}

Vamos introduzir agora uma relação de equivalência entre números virtuais:

DefiniÇÃo: Diremos que $\alpha$ está infinitamente próximo de $\beta$, ou simplesmente que $\alpha$ está próximo de $\beta$, quando a diferença entre eles for infinitesimal. Além disso, escreveremos $\alpha \approx \beta$ para indicar que $\alpha$ está próximo de $\beta$, de modo que $\alpha \approx \beta$ quando $\alpha-\beta$ for um infinitésimo.

É fácil ver que $\approx$ é uma relação simétrica e reflexiva. Para verificar sua transitividade, basta observar que:

$$
\alpha-\gamma=(\alpha-\beta)+(\beta-\gamma)
$$

$\operatorname{logo} \alpha \approx \beta$ e $\beta \approx \gamma$ implicam $\alpha \approx \gamma$, pois a soma de dois infinitésimos também é infinitesimal. Temos então que a proximidade é de fato uma relação de equivalência entre números virtuais.

Nos termos da definição acima, os infinitésimos são exatamente aqueles virtuais próximos do zero. A proposição seguinte mostra que a relação de proximidade preserva os atributos de finitude:

ProposiçÃo: Se $\alpha \approx \beta$ então:

(i) $\alpha$ é infinitesimal se e somente se $\beta$ for infinitesimal;

(ii) $\alpha$ é limitado se e somente se $\beta$ for limitado; 
Prova: Os dois itens decorrem de $\alpha=(\alpha-\beta)+\beta$, pois:

(i) a soma de dois infinitésimos é infinitesimal; e

(ii) a soma de dois limitados é limitada.

Dois números reais distintos não podem estar próximos entre si, pois a diferença entre eles, sendo um real não nulo, não pode ser um infinitésimo. Em outras palavras:

Proposição: Se $x \approx y$ então $x=y$.

Como consequência, temos que:

ProposiçÃo: Se $x \approx \alpha \approx y$ então $x=y$.

Isto é, nenhum virtual pode estar próximo, ao mesmo tempo, de dois reais distintos entre si.

Nem todo virtual está próximo de algum real, isto é, existe $\alpha \in \overline{\mathbf{R}}$ tal que $\alpha \not \approx x$, para qualquer $x \in \mathbf{R}$. Por exemplo, $\infty$ não está próximo de nenhum número real.

Também não é verdade que $\omega>\mathbf{R}$ e $\psi>\mathbf{R}$ implique em $\omega \approx \psi$. Por exemplo, $\infty>\mathbf{R}$ e $\infty^{2}>\mathbf{R}$, mas $\infty \not \varpi \infty^{2}$, pois $\infty^{2}-\infty=\infty(\infty-1)>\mathbf{R}$ não é infinitesimal. Proposição: Se $\alpha \leq \beta \leq \gamma$ e $\alpha \approx \gamma$ então $\alpha \approx \beta \approx \gamma$.

Prova: Temos em $\mathbf{R}$ que $a \leq b \leq c$ implica:

$$
|b-a| \leq|c-a| \text {. }
$$

O TEV nos garante então que $\alpha \leq \beta \leq \gamma$ implica:

$$
|\beta-\alpha| \leq|\gamma-\alpha| \text {. }
$$

Portanto, se $\gamma \approx \alpha$ então $\beta \approx \alpha$.

A adição preserva a proximidade entre números virtuais:

Proposição: Se $\alpha_{1} \approx \alpha_{2}$ e $\beta_{1} \approx \beta_{2}$ então $\alpha_{1}+\beta_{1} \approx \alpha_{2}+\beta_{2}$.

Prova: Basta observar que:

$$
\left(\alpha_{1}+\beta_{1}\right)-\left(\alpha_{2}+\beta_{2}\right)=\left(\alpha_{1}-\alpha_{2}\right)+\left(\beta_{1}-\beta_{2}\right),
$$

e lembrar que a soma de dois infinitésimos também é infinitesimal.

Corolário:se $\alpha \approx x$ e $\beta \approx y$ então $\alpha+\beta \approx x+y$.

Quanto à relação entre multiplicação e proximidade:

Proposição: Se $\lambda_{1} \approx \lambda_{2}$ e $\mu_{1} \approx \mu_{2}$, com $\lambda_{1}$ e $\mu_{1}$ limitados, então $\lambda_{1} \mu_{1} \approx \lambda_{2} \mu_{2}$.

Prova: Para qualquer quadra de virtuais $\lambda_{1}, \mu_{1}, \lambda_{2}$ e $\mu_{2}$, vale que:

$$
\lambda_{1} \mu_{1}-\lambda_{2} \mu_{2}=\lambda_{1} \mu_{1}-\lambda_{1} \mu_{2}+\lambda_{1} \mu_{2}-\lambda_{2} \mu_{2}=\lambda_{1}\left(\mu_{1}-\mu_{2}\right)+\left(\lambda_{1}-\lambda_{2}\right) \mu_{2} .
$$

Como $\lambda_{1}$ e $\mu_{2}$ são limitados, e $\left(\mu_{1}-\mu_{2}\right)$ e $\left(\lambda_{1}-\lambda_{2}\right)$ são infinitesimais, concluímos que $\left(\lambda_{1} \mu_{1}-\lambda_{2} \mu_{2}\right)$ também é infinitesimal. 
Um contra-exemplo simples com um dos fatores ilimitado é:

Neste caso temos:

$$
\lambda_{1}=1, \quad \lambda_{2}=1+\frac{1}{\infty}, \quad \text { e } \quad \mu_{1}=\mu_{2}=\infty .
$$

$$
\lambda_{1} \mu_{1}-\lambda_{2} \mu_{2}=\infty-\left(1+\frac{1}{\infty}\right) \infty=-\frac{\infty}{\infty}=-1
$$

$\operatorname{logo} \lambda_{1} \mu_{1} \not \approx \lambda_{2} \mu_{2}$

Particularizando o resultado acima, concluímos que se $\alpha \approx x$ e $\beta \approx y$ então $\alpha \beta \approx$ $x y$.

Quanto à inversão multiplicativa:

ProposiçÃo: Se $\alpha \approx x \neq 0$ então $\alpha$ é inversível e $1 / \alpha \approx 1 / x$.

Prova: Se $x>0$ então $x / 2<\alpha<2 x$, e se $x<0$ então $2 x<\alpha<x / 2$. Em ambos os casos $\alpha$ será inversível com $1 / \alpha$ limitado. Como:

$$
\frac{1}{\alpha}-\frac{1}{x}=\frac{1}{\alpha x}(x-\alpha)
$$

temos que $1 / \alpha \approx 1 / x$, pois $1 /(\alpha x)=(1 / \alpha)(1 / x)$ é limitado e $(x-\alpha)$ é infinitesimal.

Como consequência, se $\alpha \approx x \neq 0$ e $\beta \approx y$ então:

$$
\frac{\beta}{\alpha} \approx \frac{y}{x}
$$

Vejamos agora as relações básicas entre proximidade, inclusão e mistura:

ProposiçÃo: Se $\alpha \triangleleft \beta$ e $\beta \approx x$ então $\alpha \approx x$.

Prova: Seja $\mathcal{F}$ o funcional unário real dado por:

$$
\mathcal{F}(c)=|c-x| \text {. }
$$

Como $\alpha \triangleleft \beta$, temos que $\overline{\mathcal{F}}(\alpha) \triangleleft \overline{\mathcal{F}}(\beta)$, isto é:

$$
|\alpha-x| \triangleleft|\beta-x| \text {. }
$$

$\mathrm{O}$ virtual $|\beta-x|$ é infinitesimal, pois $\beta \approx x$. Logo $|\alpha-x|$ também é infinitesimal, ou seja $\alpha \approx x$.

Entretanto, não é verdade que $\alpha \triangleleft \beta \approx \gamma$ implique $\alpha \approx \gamma$, como mostra o seguinte contra-exemplo: $\alpha=\infty+1$, e $\beta=\gamma=\infty$.

ProposiçÃo: Se $\alpha$ é uma mistura de $\beta$ e $\gamma$, e $\beta \approx x \approx \gamma$, então $\alpha \approx x$.

Prova: Consideremos novamente o funcional unário real $\mathcal{F}$ usado na demonstração anterior. Como $\alpha$ é uma mistura de $\beta$ e $\gamma$, temos que $\overline{\mathcal{F}}(\alpha)$ é uma mistura de $\overline{\mathcal{F}}(\beta)$ e $\overline{\mathcal{F}}(\beta)$, isto é:

$$
|\alpha-x| \text { é uma mistura de }|\beta-x| \text { e }|\gamma-x| \text {. }
$$

Os virtuais $|\beta-x|$ e $|\gamma-x|$ são ambos infinitesimais, pois $\beta \approx x \approx \gamma$. Portanto $|\alpha-x|$ também é infinitesimal, ou seja: $\alpha \approx x$. 


\subsection{Continuidade}

Estamos supondo que todos os números reais são objetos do nosso universo de discurso $\mathcal{U}$. Portanto, uma função $f: \mathrm{D} \rightarrow \mathrm{C}$ com $\mathrm{D} \subset \mathbf{R}$ e $\mathrm{C} \subset \mathbf{R}$ pode ser considerada como um funcional unário, uma vez que $\mathrm{D} \subset \mathcal{U}$ e $\mathrm{C} \subset \mathcal{U}$. Temos então a sua extensão virtual:

$$
\bar{f}: \overline{\mathrm{D}} \rightarrow \overline{\mathrm{C}}
$$

que leva números virtuais em números virtuais.

Segundo essa definição, se $\alpha=\left\langle a_{1}, a_{2}, a_{3}, \ldots\right\rangle$ pertence a $\overline{\mathrm{D}} \subset \overline{\mathbf{R}}$, então:

$$
\bar{f}(\alpha)=\left\langle f\left(a_{1}\right), f\left(a_{2}\right), f\left(a_{3}\right), \ldots\right\rangle,
$$

onde os números reais $f\left(a_{i}\right)$ podem ser calculados a partir de um certo valor do índice $i$.

Como estamos usando letras gregas para denotar os números virtuais, e letras latinas para os números reais, o tipo de letra usada para representar o argumento na notação $\bar{f}(\alpha)$ já é suficiente para indicar que se trata da extensão virtual de $f$, e não da própria $f$. Assim, a fim de não sobrecarregar a notação, vamos omitir a barra que distingue $f$ de sua extensão virtual sempre que não houver risco de confusão.

DefiniçÃo: Seja $f: \mathrm{D} \rightarrow \mathbf{R}$ com $\mathrm{D} \subset \mathbf{R}$ e $\mathrm{C} \subset \mathbf{R}$. Diremos que $f$ é contínua em $x \in \mathrm{D}$ quando, para todo $\alpha \in \overline{\mathrm{D}}$ com $\alpha \approx x$, tivermos $f(\alpha) \approx f(x)$.

É fácil ver que essa condição é equivalente à definição usual de continuidade de $f$ em um ponto do seu domínio. Portanto a definição seguinte equivale à continuidade "pontoa-ponto" do Cálculo tradicional:

DefiniçÃo: Diremos que $f$ é contínua quando ela for contínua em todos os $x \in \mathrm{D}$.

Utilizando o TEV e os fatos sobre a proximidade estabelecidos na seção anterior, podemos demonstrar diretamente, a partir das definições acima, os resultados básicos sobre a continuidade de funções reais:

Proposição: Se $f$ e $g$ são funções reais definidas sobre um mesmo domínio $\mathrm{D} \subset \mathbf{R}$ e contínuas em $x \in \mathrm{D}$ então $f+g$ também é contínua em $x$.

Prova: Como $(f+g)(a)=f(a)+g(a)$, para todo $a \in \mathrm{D}$, o TEV nos garante que $(f+g)(\alpha)=f(\alpha)+g(\alpha)$, para todo $\alpha \in \overline{\mathrm{D}}$. Basta agora lembrarmos que $f(\alpha) \approx f(x)$ e $g(\alpha) \approx g(x)$ implicam $f(\alpha)+g(\alpha) \approx f(x)+g(x)$.

Analogamente verifica-se que:

Proposição: Se $f$ e $g$ são funções reais definidas sobre um mesmo domínio $\mathrm{D} \subset \mathbf{R}$, e contínuas em $x \in \mathrm{D}$, então $f g$ também é contínua em $x$. Além disso, se $g(x) \neq 0$ então $f / g$ também é contínua em $x$. 
O argumento intuitivo para o resultado seguinte confunde-se com sua demonstração formal:

Proposição: Se duas funções reais $f$ e $g$ são contínuas e formam uma cadeia (isto é, se o domínio de $g$ contém a imagem de $f$ ) então a função composta $(g \circ f)$ também é contínua.

Prova: Se $x \in \mathrm{D}$ e $\alpha \in \overline{\mathrm{D}} \operatorname{com} \alpha \approx x$ então $f(\alpha) \approx f(x)$, pois $f$ é contínua. Como $g$ também é contínua, temos que $g[f(\alpha)] \approx g[f(x)]$. Portanto a composta $(g \circ f)$ é uma função contínua.

Utilizando os números virtuais, podemos formular uma condição de continuidade mais restritiva do que a utilizada acima:

DEFINIÇÃO: Diremos que $f: \mathrm{D} \rightarrow \mathrm{C}$ é uniformemente contínua sobre $\mathrm{A} \subset \mathrm{D}$ quando, para quaisquer $\alpha, \beta \in \overline{\mathrm{A}} \operatorname{com} \alpha \approx \beta$, tivermos $f(\alpha) \approx f(\beta)$.

É fácil verificar que essa condição é equivalente à continuidade uniforme da Análise Matemática tradicional lembrando que os números virtuais são classes de sequências de números reais. Podemos verificar a continuidade uniforme de uma função real diretamente pela definição acima. Por exemplo:

Proposição: A função coseno é uniformemente contínua em $\mathbf{R}$.

Prova: Observamos inicialmente que:

$$
|\cos x-\cos y| \leq|x-y|
$$

para quaisquer $x$ e $y$ reais. Portanto, pelo TEV, temos que

$$
|\cos \xi-\cos v| \leq|\xi-v|
$$

para quaisquer $\xi$ e $v$ virtuais. Logo $\cos \xi \approx \cos v$ sempre que $\xi \approx v$.

\subsection{Derivação}

Para simplificar a notação, introduziremos agora uma outra relação entre números virtuais:

DEFINIÇÃO: Diremos que dois números virtuais são vizinhos quando eles estão próximos mas são dessemelhantes, isto é: $\alpha$ e $\beta$ são vizinhos quando $\alpha \approx \beta$ e $\alpha \nprec \beta$. Além disso, escreveremos $\alpha \sim \beta$ para representar o fato de $\alpha \in \overline{\mathbf{R}}$ e $\beta \in \overline{\mathbf{R}}$ serem vizinhos. 
Essa relação de vizinhança é simétrica, mas não é reflexiva nem associativa, e portanto não é uma relação de equivalência em $\overline{\mathbf{R}}$. Com essa definição, os infinitésimos inversíveis são exatamente os vizinhos do zero. A proposição seguinte exemplifica o uso da relação de vizinhança:

Proposição: Se $\varepsilon \in \overline{\mathbf{R}}$ é um infinitésimo inversível, isto é, se $\varepsilon \sim 0$, então:

$$
\frac{\operatorname{sen} \varepsilon}{\varepsilon} \approx 1
$$

Prova: Vale em $\mathbf{R}$ que:

$$
|x|<\frac{\pi}{2} \text { e } x \neq 0 \quad \text { implicam } \quad \cos x<\frac{\operatorname{sen} x}{x}<1 .
$$

Portanto, pelo TEV, vale em $\overline{\mathbf{R}}$ :

$$
|\xi|<\frac{\pi}{2} \text { e } \xi \nprec 0 \quad \text { implicam } \quad \cos \xi<\frac{\operatorname{sen} \xi}{\xi}<1 .
$$

Assim, para todo $\varepsilon \sim 0$ :

$$
\cos \varepsilon<\frac{\operatorname{sen} \varepsilon}{\varepsilon}<1
$$

Como a função coseno é contínua, temos que $\cos \varepsilon \approx \cos 0=1$, logo:

$$
\frac{\operatorname{sen} \varepsilon}{\varepsilon} \approx 1
$$

pois $\alpha<\beta<\gamma$ com $\alpha \approx \gamma$ implica $\beta \approx \gamma$.

A relação de vizinhança facilita o enunciado da condição de derivabilidade de uma função real em um ponto do seu domínio:

DefiniçÃo: Seja $f: \mathrm{D} \rightarrow \mathrm{C}$ uma função com $\mathrm{D} \subset \mathbf{R}$ e $\mathrm{C} \subset \mathbf{R}$. Diremos que $f$ é derivável em $x \in \mathrm{D}$ quando existir $m \in \mathbf{R}$ tal que, para todo $\alpha \in \overline{\mathrm{D}}$ com $\alpha \sim x$, tivermos:

$$
\frac{f(\alpha)-f(x)}{\alpha-x} \approx m
$$

Além disso, diremos simplesmente que $f$ é derivável quando ela for derivável em todos os pontos do seu domínio.

Não é difícil ver que esta condição de derivabilidade é equivalente à definição tradicional. A demonstração do resultado seguinte é o primeiro exemplo de aplicação do Lema da Semelhança, que vimos na seç. 1.9:

Proposição: Se uma função $f: \mathrm{D} \rightarrow \mathrm{C}$ é derivável em $x \in \mathrm{D}$ então $f$ é contínua neste ponto. 
Prova: Seja $\alpha \in \overline{\mathrm{D}}$ um virtual com $\alpha \approx x$. Temos três possibilidades:

(i) $\alpha=x$ : neste caso temos $f(\alpha)=f(x), \log 0 \quad f(\alpha) \approx f(x)$.

(ii) $\alpha \sim x$ : neste caso temos $\alpha \nsim x$, e portanto $(\alpha-x)$ é um virtual inversível:

$$
f(\alpha)-f(x)=\frac{f(\alpha)-f(x)}{\alpha-x} \cdot(\alpha-x) \approx m .0=0 .
$$

Ou seja: $f(\alpha) \approx f(x)$.

(iii) $\alpha \neq x$ com $\alpha \asymp x$ : neste caso não podemos fazer como no item anterior, pois o virtual $(\alpha-x)$ não é inversível. Entretanto, pelo Lema da Semelhança, existe um objeto virtual $\gamma$ tal que $x$ e $\gamma$ decompõem $\alpha$. De $\gamma \triangleleft \alpha$ decorre $\gamma \approx x$ (seç. 2.3). Por outro lado, $\gamma \nprec x$, logo $f(\gamma) \approx f(x)$, pelo mesmo argumento usado no item (ii) acima. Como $f(\alpha)$ é uma mistura de $f(x)$ e $f(\gamma)$ (seç. 1.8), concluímos que $f(\alpha) \approx f(x)$.

Como corolário da demonstração acima, temos que a condição $\alpha \approx x$ da definição de continuidade equivale a $\alpha \sim x$. Isto é:

Proposição: Uma função $f: \mathrm{D} \rightarrow \mathrm{C}$ é contínua em $x \in \mathrm{D}$ se e somente se $f(\alpha) \approx f(x)$, para todo $\alpha \in \overline{\mathrm{D}}$ com $\alpha \sim x$.

A função derivada pode ser introduzida do seguinte modo:

DEFINiÇÃo: Se $f: \mathrm{D} \rightarrow \mathrm{C}$ é uma função derivável então, para cada $x \in \mathrm{D}$, existe apenas um $m_{x} \in \mathbf{R}$ que satisfaz a condição de derivabilidade neste ponto. Chamaremos de derivada de $f$ a função real $f^{\prime}$ dada por:

$$
\begin{aligned}
f^{\prime}: \mathrm{D} & \rightarrow \mathbf{R} \\
x & \mapsto m_{x} .
\end{aligned}
$$

A fim de evitar o excesso de tecnicalidades, vamos restringir o estudo das derivadas a pontos interiores ao domínio da função:

DefiniçÃo: Seja $\mathrm{D}$ um subconjunto qualquer de $\mathbf{R}$. Diremos que $x \in \mathbf{R}$ é um ponto interior a $\mathrm{D}$ quando $\alpha \approx x$ implicar $\alpha \in \overline{\mathrm{D}}$.

Nos exemplos seguintes, usaremos a letra $x$ para representar a variável independente de uma função, e o símbolo $d x$ para indicar uma variação infinitesimal inversível de $x$, isto é, um número virtual $d x \sim 0$. Se $x$ é um ponto interior ao domínio de uma função $f$, então $f$ é derivável em $x$ quando os quocientes:

$$
\frac{f(x+d x)-f(x)}{d x}
$$

estão infinitamente próximo de um mesmo real, para qualquer $d x \sim 0$. 
(i) Se $f(x)=k$, para todo $x \in \mathbf{R}$, então, para qualquer $d x \sim 0$ :

$$
\frac{f(x+d x)-f(x)}{d x}=\frac{k-k}{d x}=0 .
$$

Portanto toda função constante é derivável, e tem por derivada a função identicamente nula.

(ii) Se $f$ é a função identidade, então, para qualquer $d x \sim 0$ :

$$
\frac{(x+d x)-x}{d x}=\frac{d x}{d x}=1
$$

Portanto a função identidade é derivável e $f^{\prime}(x)=1$, para todo $x \in \mathbf{R}$.

(iii) Seja $f(x)=\operatorname{sen} x$. Pelo TEV, temos que:

$$
\operatorname{sen} \alpha-\operatorname{sen} \beta=2 \operatorname{sen} \frac{\alpha-\beta}{2} \cos \frac{\alpha+\beta}{2},
$$

para quaisquer virtuais $\alpha$ e $\beta$. Logo, para qualquer $d x \sim 0$ :

$$
\begin{aligned}
\frac{\operatorname{sen}(x+d x)-\operatorname{sen} x}{d x} & =\frac{1}{d x} 2 \operatorname{sen} \frac{d x}{2} \cos \frac{2 x+d x}{2} \\
& =\frac{\operatorname{sen}(d x / 2)}{(d x / 2)} \cos \left(x+\frac{d x}{2}\right) \\
& \approx \cos x
\end{aligned}
$$

Portanto a função seno é derivável e sua derivada é a função coseno.

Utilizando o TEV e os fatos já estabelecidos sobre proximidade e continuidade, podemos demonstrar diretamente as regras de derivação usuais:

Proposição: Se $f$ e $g$ são funções reais deriváveis em um ponto $x$ interior ao domínio de ambas, então:

(i) $(f+g)$ também é derivável em $x$ e $(f+g)^{\prime}(x)=f^{\prime}(x)+g^{\prime}(x)$; e

(ii) $(f g)$ também é derivável em $x$ e $(f g)^{\prime}(x)=f^{\prime}(x) g(x)+f(x) g^{\prime}(x)$.

Prova: (i) Para todo $d x \sim 0$ :

$$
\begin{aligned}
\frac{(f+g)(x+d x)-(f+g)(x)}{d x} & =\frac{f(x+d x)+g(x+d x)-f(x)-g(x)}{d x} \\
& =\frac{f(x+d x)-f(x)}{d x}+\frac{g(x+d x)-g(x)}{d x} \\
& \approx f^{\prime}(x)+g^{\prime}(x) .
\end{aligned}
$$


(ii) Para qualquer $d x \sim 0$ :

$$
\begin{aligned}
\frac{(f g)(x+}{d x)-(f g)(x)} & = \\
& =\frac{f(x+d x) g(x+d x)-f(x) g(x+d x)+f(x) g(x+d x)-f(x) g(x)}{d x} \\
& =\frac{f(x+d x)-f(x)}{d x} g(x+d x)+f(x) \frac{g(x+d x)-g(x)}{d x} \\
& \approx f^{\prime}(x) g(x)+f(x) g^{\prime}(x),
\end{aligned}
$$

onde usamos que $g(x+d x) \approx g(x)$, isto é, que $g$ é contínua em $x$.

Proposição: Se $g: \mathrm{D} \rightarrow \mathbf{R}$ é uma função derivável em um ponto $x$ interior ao seu domínio e $g(x) \neq 0$, então $(1 / g)$ é derivável em $x$ e:

$$
\left(\frac{1}{g}\right)^{\prime}(x)=-\frac{g^{\prime}(x)}{[g(x)]^{2}}
$$

Prova: Para qualquer $d x \sim 0$, o número virtual $g(x+d x)$ é inversível, pois, sendo $g$ contínua em $x$, temos $g(x+d x) \approx g(x) \neq 0$. Basta agora observar que:

$$
\frac{\frac{1}{g(x+d x)}-\frac{1}{g(x)}}{d x}=-\frac{g(x+d x)-g(x)}{d x} \frac{1}{g(x+d x) g(x)} \approx-\frac{g^{\prime}(x)}{[g(x)]^{2}} .
$$

A demonstração da regra da cadeia utiliza o Lema da Disjunção:

Proposição: Se $f: \mathrm{D} \rightarrow \mathrm{C}$ é uma função real derivável em $x \in \mathrm{D}$, e $g: \mathrm{C} \rightarrow \mathbf{R}$ uma função real derivável em $y=f(x)$, então a função composta $(g \circ f)$ também é derivável em $x \in \mathrm{D}$ e:

$$
(g \circ f)^{\prime}(x)=g^{\prime}[f(x)] f^{\prime}(x)
$$

Prova: Devemos mostrar que, para qualquer $\alpha \in \overline{\mathrm{D}}$ com $\alpha \sim x$ :

$$
\frac{g[f(\alpha)]-g[f(x)]}{\alpha-x} \approx g^{\prime}[f(x)] f^{\prime}(x) .
$$

Como $f$ é contínua em $x$, temos $f(\alpha) \approx f(x)$. Há portanto três possibilidades:

(i) $f(\alpha)=f(x)$ : neste caso temos $f^{\prime}(x)=0$ e:

$$
\frac{g[f(\alpha)]-g[f(x)]}{\alpha-x}=0 .
$$

(ii) $f(\alpha) \sim f(x)$ : neste caso temos $\alpha \nprec x$, e portanto $[f(\alpha)-f(x)]$ é um virtual inversível:

$$
\frac{g[f(\alpha)]-g[f(x)]}{\alpha-x}=\frac{g[f(\alpha)]-g[f(x)]}{f(\alpha)-f(x)} \frac{f(\alpha)-f(x)}{\alpha-x} \approx g^{\prime}[f(x)] f^{\prime}(x) .
$$


(iii) $f(\alpha) \neq f(x)$ com $f(\alpha) \asymp f(x)$ : neste caso não podemos fazer como no item anterior, pois o virtual $[f(\alpha)-f(x)]$ não é inversível. Consideremos então os atributos:

$$
\mathcal{P}(a): \quad a \in \mathrm{D} \quad \text { e } \quad f(\alpha)=f(x)
$$

e

$$
\mathcal{Q}(a): \quad a \in \mathrm{D} \quad \text { e } \quad f(\alpha) \neq f(x)
$$

O virtual $\alpha \in \overline{\mathrm{D}}$ satisfaz a extensão da disjunção destes dois atributos, mas não satisfaz $\overline{\mathcal{P}}$ nem $\overline{\mathcal{Q}}$. Portanto, pelo Lema da Disjunção (seç. 1.9), existe uma decomposição de $\alpha$ em dois objetos virtuais $\beta$ e $\gamma$ tais que $\overline{\mathcal{P}}(\beta)$ e $\overline{\mathcal{Q}}(\gamma)$, isto é:

$$
f(\beta)=f(x) \quad \text { e } \quad f(\gamma) \nprec f(x) .
$$

De $f(\beta)=f(x)$ decorre $f^{\prime}(x)=0$ e então:

$$
\frac{g[f(\beta)]-g[f(x)]}{\beta-x}=0=g^{\prime}[f(x)] f^{\prime}(x) .
$$

Por outro lado, $\gamma \triangleleft \alpha \approx x$ implica $\gamma \approx x$ (seç. 2.3), consequentemente $f(\gamma) \approx f(x)$. Como, além disso, $f(\gamma) \nprec f(x)$, concluímos que $f(\gamma) \sim f(x)$. Temos então, pelo mesmo argumento usado no item (ii) acima, que:

$$
\frac{g[f(\gamma)]-g[f(x)]}{\gamma-x} \approx g^{\prime}[f(x)] f^{\prime}(x)
$$

Para finalizar a demonstração, basta observar que o número virtual:

$$
\frac{g[f(\alpha)]-g[f(x)]}{\alpha-x}
$$

é uma mistura de

$$
\frac{g[f(\beta)]-g[f(x)]}{\beta-x} \quad \text { e } \quad \frac{g[f(\gamma)]-g[f(x)]}{\gamma-x}
$$

que estão, ambos, próximos de $g^{\prime}[f(x)] f^{\prime}(x)$, e concluir então (seç. 2.3) que:

$$
\frac{g[f(\alpha)]-g[f(x)]}{\alpha-x} \approx g^{\prime}[f(x)] f^{\prime}(x)
$$




\subsection{Quantidades Virtuais}

Ao construirmos a extensão virtual de um universo de discurso matemático, consideramos apenas predicados e funcionais finitários, sendo esta restrição indispensável para a validade do TEV na forma em que ele foi enunciado. Por outro lado, em muitas ocasiões precisamos tratar de uma quantidade ilimitada de objetos virtuais. A construção da integral definida de uma função real como uma soma de uma quantidade ilimitada de infinitésimos é um exemplo típico. A fim de superar esta dificuldade, vamos introduzir agora a noção de quantidade virtual de objetos virtuais.

DefiniÇÃo: Chamaremos uma $n$-upla simplesmente de upla quando não quisermos explicitar seu número de entradas. Em outras palavras, um objeto matemático é uma upla quando existe $n \in \mathbf{N}$ tal que este objeto é uma $n$-upla.

DEFIniÇÃo: Diremos que uma upla é uma upla-objeto quando ela e todas as suas entradas forem objetos de $\mathcal{U}$. Em outras palavras, uma $n$-upla $a=\left(a_{1}, a_{2}, \ldots, a_{n}\right)$ é uma uplaobjeto quando $a \in \mathcal{U}$ e $a_{i} \in \mathcal{U}$, para $i=1, \ldots, n$.

DEFINIÇÃo: Seja $\mathcal{P} \subset \mathcal{U}$ atributo dado por:

$$
\mathcal{P}(a): \quad a \text { é uma upla-objeto. }
$$

A extensão virtual deste predicado define o conceito de upla virtual:

$$
\overline{\mathcal{P}}(\alpha): \quad \alpha \text { é uma upla virtual. }
$$

DefiniçÃo: Consideremos o funcional unário $\mathcal{F}$ que devolve o número de entradas de cada upla-objeto:

$$
\mathcal{F}(a)=n, \quad \text { se } a=\left(a_{1}, a_{2}, \ldots, a_{n}\right) .
$$

A extensão virtual deste predicado define o número de entradas de cada upla virtual:

$$
\overline{\mathcal{F}}(\alpha)=\nu,
$$

neste caso, diremos que $\alpha$ é uma $\nu$-upla virtual.

Segundo estas definições, uma $\infty$-upla virtual é uma classe do tipo:

$$
\langle(a),(b, c),(d, e, f), \ldots\rangle \in \overline{\mathcal{U}}
$$

onde $a, b, c, \ldots$ são objetos de $\mathcal{U}$. É importante observar que uma $\infty$-upla não é simplesmente uma sequência infinita de objetos reais, mas uma classe de equivalência de 
$n$-uplas. Assim, temos também, por exemplo, $(\infty+1)$-uplas, que são objetos virtuais do tipo:

$$
\langle(a, b),(c, d, e),(f, g, h, i), \ldots\rangle
$$

Não há risco de confusão ao omitirmos (como fizemos acima) o adjetivo "virtual" quando nos referirmos a " $\nu$-uplas" ou " $\infty$-uplas", uma vez que os símbolos $\nu$ e $\infty$ indicam claramente que se trata de um objeto virtual.

É habitual representarmos $n$-uplas escrevendo $\left(a_{1}, a_{2}, \ldots, a_{n}\right)$. Analogamente, indicaremos $\nu$-uplas virtuais por $\left(\alpha_{1}, \alpha_{2}, \ldots, \alpha_{\nu}\right)$. Por exemplo, uma $\infty$-upla poderá ser representada por $\left(\alpha_{1}, \alpha_{2}, \ldots, \alpha_{\infty}\right)$.

Também é usual indicarmos a $i$-ésima entrada da $n$-upla $\left(a_{1}, a_{2}, \ldots, a_{n}\right)$ por $a_{i}$, onde $i$ é um indice que varia entre 1 e $n$. Analogamente, indicaremos a $\iota$-ésima entrada virtual da $\nu$-upla virtual $\left(\alpha_{1}, \alpha_{2}, \ldots, \alpha_{\nu}\right)$ por $\alpha_{\iota}$, e diremos que a letra grega $\iota$ é um indice virtual. Mais precisamente:

DefiniçÃo: Seja $\mathcal{F}$ o funcional binário que seleciona a $i$-ésima entrada de uma $n$-uplaobjeto, para cada $i \in \mathbf{N}$ tal que $1 \leq i \leq n$ :

$$
\mathcal{F}\left[\left(a_{1}, a_{2}, \ldots, a_{n}\right), i\right]=a_{i}
$$

A extensão virtual deste funcional binário define a $\iota$-ésima entrada virtual de uma $\nu$-upla virtual, para cada natural virtual $\iota \in \overline{\mathbf{N}}$ tal que $1 \leq \iota \leq \nu$ :

$$
\overline{\mathcal{F}}\left[\left(\alpha_{1}, \alpha_{2}, \ldots, \alpha_{\nu}\right), \iota\right]=\alpha_{\iota}
$$

As entradas virtuais, como definidas acima, são tais que:

ProposiçÃo: Uma $\nu$-upla virtual é igual a uma $\kappa$-upla virtual se e somente se $\nu=\kappa$ e, além disso, elas têm as mesmas entradas virtuais. Em outras palavras:

$$
\left(\alpha_{1}, \alpha_{2}, \ldots, \alpha_{\nu}\right)=\left(\beta_{1}, \beta_{2}, \ldots, \beta_{\kappa}\right)
$$

se e somente se $\nu=\kappa$ e $\alpha_{\iota}=\beta_{\iota}$, para todo $\iota \leq \nu$.

Prova: Basta aplicar o TEV ao fato básico sobre a noção de $n$-upla:

$$
\left(a_{1}, a_{2}, \ldots, a_{n}\right)=\left(b_{1}, b_{2}, \ldots, b_{k}\right)
$$

se e somente se $n=k$ e $a_{i}=b_{i}$, para todo $i \leq n$.

É importante observar, entretanto, que uma $\nu$-upla virtual não é o mesmo que uma função definida do conjunto:

$$
\{\iota \in \overline{\mathbf{N}} \mid 1 \leq \iota \leq \nu\}
$$


a valores em $\overline{\mathcal{U}}$, pois algumas destas funções não estão associadas a nenhuma $\nu$-upla virtual pela correspondência acima. Um contra-exemplo trivial é a função:

$$
f:\{\iota \in \overline{\mathbf{N}} \mid 1 \leq \iota \leq 2\} \rightarrow \overline{\mathbf{R}}
$$

dada por:

$$
f(\iota)= \begin{cases}1 & , \text { se } \iota=1 \\ 0 & , \text { caso contrário. }\end{cases}
$$

Não é difícil ver que se $f(\iota)$ fosse, para cada $\iota \leq 2$, a $\iota$-ésima entrada (virtual) da 2 -upla virtual

$$
\left\langle\left(a_{1}^{1}, a_{2}^{1}\right),\left(a_{1}^{2}, a_{2}^{2}\right),\left(a_{1}^{3}, a_{2}^{3}\right), \ldots\right\rangle
$$

então existiria $k \in \mathbf{N}$ tal que $a_{1}^{i}=1$ para todo $i>k$. Mas então teríamos

$$
f(\langle 1,2,1,2,1,2, \ldots\rangle) \neq 0
$$

o que não ocorre com a função $f$ definida acima.

Assim, devemos sempre entender as uplas virtuais como classes de equivalência de uplas-objeto, e não como simples coleções indexadas por um índice virtual.

A indexação virtual, como definida acima, é a chave para trabalharmos com quantidades virtuais de objetos de um certo tipo:

DEFINIÇÃO: Uma quantidade virtual de objetos de um certo tipo (isto é, que satisfazem um certo atributo virtual) é uma upla virtual cujas entradas (virtuais) são todas objetos virtuais deste tipo (isto é, que satisfazem este atributo virtual).

Vamos supor, daqui por diante, que não apenas os números reais, mas também que todas as uplas de números reais são objetos do universo de discurso fixado no início do processo de extensão virtual. Isto é: todas as $n$-uplas de números reais pertencem a $\mathcal{U}$, para todo $n \in \mathbf{N}$. Exemplificando a definição acima: uma quantidade virtual de números virtuais é uma $\nu$-upla $\left(\alpha_{1}, \alpha_{2}, \ldots, \alpha_{\nu}\right)$ tal que $\alpha_{\iota} \in \overline{\mathbf{R}}$, para todo $\iota \leq \nu$.

É importante observar que na definição de quantidade virtual não supomos que o tipo dos objetos virtuais esteja caracterizado por um atributo transferido. Por exemplo, o atributo virtual "ser infinitesimal" não é um atributo transferido, mas podemos dizer que uma quantidade virtual de infinitésimos é uma $\nu$-upla $\left(\alpha_{1}, \alpha_{2}, \ldots, \alpha_{\nu}\right)$ tal que $\alpha_{\iota} \in \overline{\mathbf{R}}$ é infinitesimal, para todo $\iota \leq \nu$.

Ao trabalhar com $\nu$-uplas de virtuais, procuraremos usar uma notação semelhante à utilizada para $n$-uplas, com a qual já estamos bem familiarizados. Por exemplo, ao escrever:

$$
(1,2,3, \ldots, \infty)
$$


sugerimos que $\alpha_{\iota}=\iota$, para todo $\iota \leq \infty$. Ou seja, estamos nos referindo à $\infty$-upla:

$$
\langle(1),(1,2),(1,2,3), \ldots\rangle \in \overline{\mathcal{U}}
$$

Analogamente, usaremos a notação:

$$
\underbrace{(1,1, \ldots, 1)}_{\infty \text { vezes }}
$$

que sugere $\alpha_{\iota}=1$, para todo $\iota \leq \infty$, para indicar a $\infty$-upla:

$$
\langle(1),(1,1),(1,1,1), \ldots\rangle \in \overline{\mathcal{U}}
$$

Exemplo adicional: escrever

$$
\underbrace{(\infty, \infty, \ldots, \infty)}_{\infty \text { vezes }}
$$

sugere que $\alpha_{\iota}=\infty$, para todo $\iota \leq \infty$. Assim, usaremos esta notação para denotar a $\infty$-upla:

$$
\langle(1),(2,2),(3,3,3), \ldots\rangle \in \overline{\mathcal{U}}
$$

Não é difícil estender operações de adição e multiplicação para quantidades virtuais de operandos:

DEFInIÇÃO: Seja:

$$
\mathcal{F}\left[\left(a_{1}, a_{2}, \ldots, a_{n}\right)\right]=a_{1}+a_{2}+\cdots+a_{n}
$$

o funcional unário que soma as entradas de qualquer upla de números reais. A extensão virtual deste funcional define a soma de qualquer quantidade virtual de números virtuais:

$$
\overline{\mathcal{F}}\left[\left(\alpha_{1}, \alpha_{2}, \ldots, \alpha_{\nu}\right)\right]=\alpha_{1}+\alpha_{2}+\cdots+\alpha_{\nu}
$$

Cabe ressaltar que, nessa definição, $\mathcal{F}$ é um funcional unário.

Alguns exemplos:

(i) É claro que

$$
\underbrace{1+1+1+\cdots+1}_{n \text { vezes }}=n
$$

para qualquer $n \in \mathbf{N}$. Portanto, pelo TEV, temos que:

$$
\underbrace{1+1+1+\cdots+1}_{\nu \text { vezes }}=\nu
$$


para todo $\nu \in \overline{\mathbf{N}}$, sendo que o membro esquerdo dessa igualdade representa a soma (virtual) das entradas da $\nu$-upla:

$$
\underbrace{(1,1, \ldots, 1)}_{\nu \text { vezes }} \in \overline{\mathcal{U}} .
$$

Em particular, tomando $\nu=\infty$, obtemos:

$$
\underbrace{1+1+1+\cdots+1}_{\infty \text { vezes }}=\infty
$$

(ii) Pelo TEV temos, para todo $\nu \in \overline{\mathbf{N}}$, que:

$$
1+2+3+\cdots+\nu=\frac{\nu(\nu+1)}{2} .
$$

Portanto, tomando $\nu=\infty$, obtemos:

$$
1+2+3+\cdots+\infty=\frac{\infty(\infty+1)}{2}
$$

Procedendo de forma análoga, podemos definir o produto:

$$
\alpha_{1} \alpha_{2} \cdots \alpha_{\nu} \in \overline{\mathbf{R}}
$$

de qualquer quantidade virtual $\left(\alpha_{1}, \alpha_{2}, \ldots, \alpha_{\nu}\right)$ de números virtuais, bem como outras operações algébricas. Muitas vezes há mais de uma forma de se estender uma operação aritmética ou algébrica, sendo que o TEV nos garante que elas são equivalentes, como no exemplo seguinte:

(iii) Podemos estender a operação unária que calcula o fatorial de um número natural de duas formas: pela extensão virtual do funcional unário:

$$
\mathcal{F}(n)=n !
$$

ou diretamente pelo produto virtual:

$$
\nu !=1.2 .3 \ldots(\nu-1) \nu .
$$

Entretanto, como

$$
n !=1.2 .3 \ldots(n-1) n, \quad \text { para qualquer } n \in \mathbf{N} \text {, }
$$

o TEV mostra que

$$
\nu !=1.2 .3 \ldots(\nu-1) \nu, \quad \text { para qualquer } \nu \in \overline{\mathbf{N}} \text {. }
$$


Em ambos os casos:

$$
\text { se } \quad \nu=\left\langle n_{1}, n_{2}, n_{3}, \ldots\right\rangle \quad \text { então } \quad \nu !=\left\langle n_{1} !, n_{2} !, n_{3} !, \ldots\right\rangle \text {, }
$$

onde os fatoriais podem ser calculados a partir de um certo $n_{i}$. Em particular:

$$
\infty !=1.2 .3 \ldots(\infty-1) . \infty=\langle 1,2,6,24,120, \ldots\rangle \in \overline{\mathbf{N}}
$$

É conveniente manter, sempre que possível, as notações e convenções sintáticas universalmente aceitas para quantidades reais. Como ilustram os exemplos abaixo:

(iv) Tendo estendido a operação de potenciação para os números virtuais, obtemos:

$$
\underbrace{\infty . \infty . \infty \ldots \infty}_{(\infty+1) \text { vezes }}=\infty^{(\infty+1)}=\infty^{\infty} \infty
$$

ou ainda:

$$
\underbrace{3.3 .3 \ldots 3}_{\infty^{2} \text { vezes }}=3^{\infty^{2}}
$$

onde mantivemos a convenção usual:

$$
3^{\infty^{2}}=3^{\left(\infty^{2}\right)} \neq\left(3^{\infty}\right)^{2}=3^{\infty} \cdot 3^{\infty}
$$

(v) Após definir os coeficientes binomiais virtuais (o TEV nos garante que todas as definições óbvias são equivalentes), podemos escrever a fórmula do binmio de Newton para potências virtuais:

$$
(\alpha+\beta)^{\nu}=\alpha^{\nu}+\nu \alpha^{\nu-1} \beta+\cdots+\left(\begin{array}{l}
\nu \\
\iota
\end{array}\right) \alpha^{\nu-\iota} \beta^{\iota}+\cdots+\nu \alpha \beta^{\nu-1}+\beta^{\nu} .
$$

Temos então, em particular:

$$
(\alpha+\beta)^{\infty}=\alpha^{\infty}+\infty \alpha^{\infty-1} \beta+\cdots+\left(\begin{array}{c}
\infty \\
\iota
\end{array}\right) \alpha^{\infty-\iota} \beta^{\iota}+\cdots+\infty \alpha \beta^{\infty-1}+\beta^{\infty} .
$$

A adição virtual:

$$
\underbrace{\frac{1}{\infty}+\frac{1}{\infty}+\frac{1}{\infty}+\cdots+\frac{1}{\infty}}_{\infty \text { vezes }}=\infty \frac{1}{\infty}=1
$$

mostra que a soma de uma quantidade ilimitada de infinitésimos pode não ser infinitesimal. Entretanto: 
Proposição: A soma de uma quantidade limitada de infinitésimos é infinitesimal.

Prova: Seja $F$ o funcional unário que seleciona a maior entrada de cada $n$-upla de números reais:

$$
\mathcal{F}\left[\left(a_{1}, a_{2}, \ldots, a_{n}\right)\right]=\operatorname{máx} a_{i} .
$$

A extensão deste funcional define a entrada máxima:

$$
\overline{\mathcal{F}}\left[\left(\alpha_{1}, \alpha_{2}, \ldots, \alpha_{\nu}\right)\right]=\operatorname{máx} \alpha_{\iota} \in \overline{\mathbf{R}}
$$

de qualquer $\nu$-upla de virtuais.

É claro que vale em $\mathbf{R}$, para qualquer $n$-upla $\left(e_{1}, e_{2}, \ldots, e_{n}\right)$ :

$$
\left|e_{1}+e_{2}+\cdots+e_{n}\right| \leq\left|e_{1}\right|+\left|e_{2}\right|+\cdots+\left|e_{n}\right| \leq n \text { máx }\left|e_{i}\right| .
$$

Portanto, pelo TEV, temos:

$$
\left|\varepsilon_{1}+\varepsilon_{2}+\cdots+\varepsilon_{\nu}\right| \leq \nu \operatorname{máx}\left|\varepsilon_{\iota}\right|,
$$

para qualquer $\nu$-upla virtual $\left(\varepsilon_{1}, \varepsilon_{2}, \ldots, \varepsilon_{\nu}\right)$ de números virtuais.

Mas se todos os $\varepsilon_{\iota}(\iota \leq \nu)$ são infinitesimais então máx $\left|\varepsilon_{\iota}\right|$ também é infinitesimal. Além disso, se $\nu$ for limitado então o produto $\nu$ máx $\left|\varepsilon_{\iota}\right|$ é um infinitésimo (seç. 2.2), logo a soma virtual $\varepsilon_{1}+\varepsilon_{2}+\cdots+\varepsilon_{\nu}$ também é infinitesimal.

\subsection{Integração}

O conceito de quantidade virtual introduzido na seção anterior nos permite construir uma linguagem cmoda para discutirmos o processo de integração de funções reais:

DefiniçÃo: Seja $[a, b] \subset \mathbf{R}$ um intervalo real fechado $(a<b)$. Diremos que uma $n$-upla $\left(x_{1}, x_{2}, \ldots, x_{n}\right)$ é uma partição do intervalo $[a, b]$ quando:

$$
a=x_{1}<x_{2}<\cdots<x_{n}<b .
$$

Neste caso, usaremos a notação $\Delta x_{i}=x_{i+1}-x_{i}$, para cada $i<n$.

Para cada intervalo $[a, b] \subset \mathbf{R}$ fixado, "ser uma partição" deste intervalo é um atributo de $n$-uplas de reais. Assim:

DefiniçÃo: Diremos que uma $\nu$-upla $\left(\xi_{1}, \xi_{2}, \ldots, \xi_{\nu}\right)$ é uma partição virtual do intervalo $[a, b]$ quando ela satisfizer a extensão virtual do atributo definido acima. Neste caso, usaremos a notação $\Delta \xi_{\iota}=\xi_{\iota+1}-\xi_{\iota}$, para cada $\iota<\nu$.

DefiniçÃo: Diremos que uma partição virtual $\left(\xi_{1}, \xi_{2}, \ldots, \xi_{\nu}\right)$ do intervalo $[a, b]$ é infinitamente fina, ou simplesmente fina, quando $\xi_{\iota} \approx \xi_{\iota+1}$, para todo $\iota<\nu$. Neste caso, usaremos a notação:

$$
d \xi_{\iota}=\xi_{\iota+1}-\xi_{\iota}, \quad \text { para cada } \iota<\nu,
$$

a fim de indicar que os virtuais $d \xi_{\iota}$ são todos infinitesimais. 
É fácil ver que todo intervalo admite uma partição fina. Por exemplo, a $\infty$-upla:

$$
\xi_{\iota}=a+\frac{\iota-1}{\infty}(b-a), \quad \text { para cada } \iota<\infty
$$

divide o intervalo $[a, b]$ em $\infty$ partes iguais:

$$
d \xi_{\iota}=\frac{(b-a)}{\infty}, \quad \text { para todo } \iota<\nu
$$

ProposiçÃo: Se uma partição fina divide um intervalo em $\nu$ partes então $\nu>\mathbf{R}$. Prova: Para qualquer partição real do intervalo $[a, b]$, temos:

$$
(b-a)=\sum_{i=1}^{n-1} \Delta x_{i} \leq(n-1) \operatorname{máx} \Delta x_{i}
$$

$\log \mathrm{O}$

$$
n \geq 1+\frac{(b-a)}{\operatorname{máx} \Delta x_{i}}
$$

O TEV nos garante então que, para qualquer partição virtual $\left(\xi_{1}, \xi_{2}, \ldots, \xi_{\nu}\right)$ do intervalo $[a, b]$ :

$$
\nu \geq 1+\frac{(b-a)}{\operatorname{máx} \Delta \xi_{\iota}} .
$$

Se esta partição é fina, então máx $\Delta \xi_{\iota}$ é um infinitésimo estritamente positivo, e portanto $\nu>\mathbf{R}$.

Com algumas definições adicionais, podemos usar a noção de partição fina para construir a integral de uma função real:

DefiniçÃo: Seja $\nu$-upla $\left(\xi_{1}, \xi_{2}, \ldots, \xi_{\nu}\right)$ uma partição fina do intervalo $[a, b]$. Diremos que uma $\nu$-upla $\left(\gamma_{1}, \gamma_{2}, \ldots, \gamma_{\nu}\right)$ é uma amostragem desta partição quando:

$$
\xi_{\iota}<\gamma_{\iota}<\xi_{\iota+1}, \quad \text { para todo } \iota<\nu
$$

Além disso, diremos que estas duas $\nu$-uplas, juntas, formam uma partição amostrada do intervalo $[a, b]$.

Não é difícil ver que toda partição fina tem uma amostragem. Podemos, por exemplo, tomar:

$$
\gamma_{\iota}=\frac{\xi_{\iota+1}+\xi_{\iota}}{2}, \quad \text { para cada } \iota<\nu
$$

Portanto, todo intervalo tem uma partição amostrada. 
Definição: Seja $f:[a, b] \rightarrow \mathbf{R}$ uma função real qualquer. Para cada partição amostrada do intervalo $[a, b]$, chamaremos o virtual:

$$
\sum_{\iota=1}^{\nu-1} f\left(\gamma_{\iota}\right) d \xi_{\iota}
$$

de soma de Riemann da função $f$, relativa a esta partição amostrada do intervalo $[a, b]$. DEFiniçÃo: Seja $f:[a, b] \rightarrow \mathbf{R}$ uma função real qualquer. Diremos que $f$ é integrável sobre o intervalo $[a, b]$ quando suas somas de Riemann relativas a todas as partições amostradas do intervalo $[a, b]$ estão próximas de um mesmo número real. Neste caso, este número será chamado de integral de $f$ sobre o intervalo $[a, b]$, e denotado por:

$$
\int_{a}^{b} f(x) d x .
$$

Não é difícil ver que esta condição de integrabilidade, e a definição correspondente de integral, equivalem àquelas devidas a Riemann.

Podemos demonstrar diretamente que toda função contínua é integrável utilizando a caracterização das funções uniformemente contínuas vista na seç. 2.4. Não faremos aqui todos os detalhes desta demonstração, mas acreditamos que o resultado seguinte seja suficiente para convencer um leitor familiarizado com as técnicas tradicionais da Análise Matemática que ela pode ser completada sem maiores dificuldades:

Proposição: Se $f:[a, b] \rightarrow \mathbf{R}$ é uma função real uniformemente contínua então as somas de Riemann relativas a duas amostragens de uma mesma partição fina do intervalo $[a, b]$ estão próximas entre si.

Prova: Se $\left(\gamma_{1}, \gamma_{2}, \ldots, \gamma_{\nu}\right)\left(\delta_{1}, \delta_{2}, \ldots, \delta_{\nu}\right)$ são duas amostragens de uma partição fina $\left(\xi_{1}, \xi_{2}, \ldots, \xi_{\nu}\right)$ do intervalo $[a, b]$, então temos:

$$
\begin{aligned}
\left|\sum_{\iota=1}^{\nu-1} f\left(\gamma_{\iota}\right) d \xi_{\iota}-\sum_{\iota=1}^{\nu-1} f\left(\delta_{\iota}\right) d \xi_{\iota}\right| & =\left|\sum_{\iota=1}^{\nu-1}\left[f\left(\gamma_{\iota}\right)-f\left(\delta_{\iota}\right)\right] d \xi_{\iota}\right| \\
& \leq \sum_{\iota=1}^{\nu-1}\left|f\left(\gamma_{\iota}\right)-f\left(\delta_{\iota}\right)\right| d \xi_{\iota} \\
& \leq \operatorname{máx}\left|f\left(\gamma_{\iota}\right)-f\left(\delta_{\iota}\right)\right| \cdot \sum_{\iota=1}^{\nu-1} d \xi_{\iota} \\
& =\operatorname{máx}\left|f\left(\gamma_{\iota}\right)-f\left(\delta_{\iota}\right)\right| \cdot(b-a) .
\end{aligned}
$$

Como $f$ é uniformemente contínua, temos que $f\left(\gamma_{\iota}\right) \approx f\left(\delta_{\iota}\right)$, para todo $\iota<\nu$, pois $\gamma_{\iota} \approx \delta_{\iota}$, para todo $\iota<\nu$. Portanto o virtual:

$$
\operatorname{máx}\left|f\left(\gamma_{\iota}\right)-f\left(\delta_{\iota}\right)\right|
$$


é um infinitésimo. Como $(b-a)$ é limitado, segue que a diferença entre somas de Riemann calculadas acima também é infinitesimal, isto é:

$$
\sum_{\iota=1}^{\nu-1} f\left(\gamma_{\iota}\right) d \xi_{\iota} \approx \sum_{\iota=1}^{\nu-1} f\left(\delta_{\iota}\right) d \xi_{\iota} .
$$




\section{Capítulo 3}

\section{Cálculo de Funções Virtuais}

Se pretendemos formalizar matematicamente processos que envolvem as construções básicas do Cálculo (como derivadas e integrais, por exemplo) então devemos definir explicitamente os processos concebidos com funções, que são os objetos aos quais estas construções se aplicam. As funções virtuais que introduzimos neste capítulo são exatamente isto: uma formalização rigorosa dos processos concebíveis com funções. Não é difícil definir estes objetos seguindo as idéias apresentadas no primeiro capítulo: basta incluir conjuntos e funções entre os objetos reais do universo de discurso inicial, tomando cuidado para que as identificações necessárias possam ser feitas com segurança.

\subsection{Conjuntos Virtuais}

Suponhamos agora que o universo de discurso matemático fixado no início do processo de extensão virtual possua conjuntos como objetos, isto é, que $\mathcal{U}$ é um conjunto que possui outros conjuntos como elementos.

DEFINIÇÃO: Um conjunto-objeto é um conjunto não-vazio de objetos de $\mathcal{U}$ que pertence, ele próprio, a $\mathcal{U}$. Em outras palavras: um conjunto-objeto é um elemento de $\mathcal{U}$ que é, ao mesmo tempo, subconjunto não-vazio de $\mathcal{U}$. Expressando esta idéia na notação simbólica da Teoria dos Conjuntos: $\mathrm{C}$ é um conjunto-objeto quando $\mathrm{C} \in \mathcal{U}$, com $\mathrm{C} \neq \varnothing$ e $\mathrm{C} \subset \mathcal{U}$. DEFINIÇÃo: Seja $\mathcal{P} \subset \mathcal{U}$ o atributo:

$$
\mathcal{P}(\mathrm{C}): \quad \quad \mathrm{C} \text { é um conjunto-objeto. }
$$

A extensão virtual deste atributo define os conjuntos virtuais:

$$
\overline{\mathcal{P}}(\Gamma): \quad \quad \Gamma \text { é um conjunto virtual. }
$$


Deve estar claro que conjuntos virtuais são classes de equivalência (pela relação "terminam iguais") de sequências de objetos reais (isto é, de elementos de $\mathcal{U}$ ). Um objeto virtual

$$
\Gamma=\left\langle\mathrm{C}_{1}, \mathrm{C}_{2}, \mathrm{C}_{3}, \ldots\right\rangle
$$

é um conjunto virtual quando, a partir de um certo valor do índice, todos os $\mathrm{C}_{i}$ forem conjuntos-objeto.

DEFINIÇÃO: A relação de pertinência entre objetos e conjuntos-objeto pode ser considerada como um predicado binário:

$$
\mathcal{P}(a, \mathrm{C}): \quad a \in \mathrm{C}
$$

Chamaremos a extensão virtual deste predicado binário de pertinência virtual:

$$
\overline{\mathcal{P}}(\alpha, \Gamma): \quad \alpha \bar{\in} \Gamma
$$

e diremos que conjuntos virtuais possuem elementos virtuais.

Segundo esta definição, um objeto virtual

$$
\alpha=\left\langle a_{1}, a_{2}, a_{3}, \ldots\right\rangle
$$

pertence virtualmente a um conjunto virtual

$$
\Gamma=\left\langle\mathrm{C}_{1}, \mathrm{C}_{2}, \mathrm{C}_{3}, \ldots\right\rangle
$$

quando existe $k \in \mathbf{N}$ tal que $a_{i} \in \mathrm{C}_{i}$, para todo $i>k$.

Pode ocorrer do conjunto vazio ser um objeto de $\mathcal{U}$, isto é: $\varnothing \in \mathcal{U}$. Entretanto, segundo a definição acima, ele não é um conjunto-objeto. Portanto o objeto virtual $\bar{\varnothing}=$ $\langle\varnothing, \varnothing, \varnothing, \ldots\rangle$ não é um conjunto virtual. Também é fácil ver que:

Proposição: Se $\Gamma$ é um conjunto virtual então $\Gamma \nsim \bar{\varnothing}$.

A definição de conjunto-objeto foi escolhida de forma a excluir os objetos virtuais semelhantes ao $\bar{\varnothing}$ da categoria de conjuntos virtuais. Isto foi feito propositadamente, a fim de preservar o princípio de extensionalidade dos conjuntos virtuais com relação à pertinência virtual: um conjunto virtual está univocamente caracterizado pelos seus elementos virtuais. Introduzimos a notação seguinte para discutir mais detalhadamente este ponto:

DEFINiÇÃo: Se $\Gamma$ é um conjunto virtual, representaremos por $[\Gamma]$ a coleção dos seus elementos virtuais, isto é:

$$
[\Gamma]=\{\alpha \in \overline{\mathcal{U}} \mid \alpha \bar{\in} \Gamma\}
$$

Temos então: 
Proposição: Os conjuntos virtuais $\Gamma$ e $\Delta$ são iguais quando têm exatamente os mesmos elementos virtuais, isto é:

$$
\Gamma=\Delta \quad \text { se e somente se } \quad[\Gamma]=[\Delta]
$$

Prova: É claro que se $\Gamma=\Delta$ então $[\Gamma]=[\Delta]$. Para provar a recíproca, suponhamos que $\Gamma \neq \Delta$, com

$$
\Gamma=\left\langle\mathrm{C}_{1}, \mathrm{C}_{2}, \mathrm{C}_{3}, \ldots\right\rangle \quad \text { e } \quad \Delta=\left\langle\mathrm{D}_{1}, \mathrm{D}_{2}, \mathrm{D}_{3}, \ldots\right\rangle .
$$

Para todo $k \in \mathbf{N}$, existe $i>k$ tal que $\mathrm{C}_{i} \neq \mathrm{D}_{i}$, ou seja, para todo $k \in \mathbf{N}$, existem $i>k$ e $a_{i} \in \mathcal{U}$ tais que:

$$
a_{i} \in \mathrm{C}_{i} \quad \text { com } \quad a_{i} \notin \mathrm{D}_{i}
$$

ou

$$
a_{i} \notin \mathrm{C}_{i} \quad \text { com } \quad a_{i} \in \mathrm{D}_{i} .
$$

Portanto, pelo menos uma das duas condições abaixo é verdadeira:

(i) para todo $k \in \mathbf{N}$, existem $i>k$ e $a_{i} \in \mathcal{U}$ tais que $a_{i} \in \mathrm{C}_{i}$ e $a_{i} \notin \mathrm{D}_{i}$;

(ii) para todo $k \in \mathbf{N}$, existem $i>k$ e $a_{i} \in \mathcal{U}$ tais que $a_{i} \notin \mathrm{C}_{i}$ e $a_{i} \in \mathrm{D}_{i}$.

Se (i) for verdadeira então existe $\alpha \bar{\in} \Gamma$ tal que não vale $\alpha \bar{\in} \Delta$, pois $\Gamma \nprec \bar{\varnothing}$. Por outro lado, se (ii) for verdadeira então existe $\alpha \in \Delta$ tal que não vale $\alpha \bar{\epsilon} \Gamma$, pois $\Delta \nsucc \bar{\varnothing}$. Em ambos os casos temos $[\Gamma] \neq[\Delta]$.

Esse resultado nos mostra que podemos identificar, com segurança, cada conjunto virtual com a coleção dos seus elementos virtuais, isto é, podemos considerar $\Gamma=[\Gamma]$. Devemos nos lembrar, entretanto, que nem toda coleção de objetos virtuais é um conjunto virtual segundo a identificação acima, isto é, nem todo conjunto real de objetos virtuais pode ser escrito na forma $[\Gamma]$, com $\Gamma$ sendo um conjunto virtual. Por exemplo:

Proposição: Se $\alpha$ e $\beta$ são dois objetos virtuais distintos então a coleção $\{\alpha, \beta\}$ constituída exatamente por estes dois elementos não é um conjunto virtual.

Prova: Seja $\alpha=\left\langle a_{1}, a_{2}, a_{3}, \ldots\right\rangle$ e $\beta=\left\langle b_{1}, b_{2}, b_{3}, \ldots\right\rangle$. Construímos então uma sequência $\left(c_{1}, c_{2}, c_{3}, \ldots\right)$ de objetos reais fazendo:

$$
c_{i}=\left\{\begin{array}{lll}
a_{i} & , \text { se } & a_{i}=b_{i} \\
b_{i} & , \text { se } & a_{i} \neq b_{i}
\end{array}\right.
$$

Como $\alpha \neq \beta$, para todo $k \in \mathbf{N}$ existe $i>k$ tal que $a_{i} \neq b_{i}$. Portanto o objeto virtual $\gamma=\left\langle c_{1}, c_{2}, c_{3}, \ldots\right\rangle$ é distinto tanto de $\alpha$ quanto de $\beta$. Mas se a coleção $\{\alpha, \beta\}$ fosse da forma $[\Gamma]$, com $\Gamma$ sendo um conjunto virtual, então $\gamma$ também pertenceria a $\{\alpha, \beta\}$. 
Tendo feito a identificação acima, passaremos a considerar os conjuntos virtuais como um tipo especial de coleção de objetos virtuais. Assim, omitiremos, daqui por diante, a barra que distingue a pertinência virtual $\bar{\epsilon} d a$ pertinência real $\in$. Diremos simplesmente que um objeto virtual $\alpha$ pertence a um conjunto virtual $\Gamma$, e escreveremos $\alpha \in \Gamma$ ao invés de $\alpha \bar{\in} \Gamma$. Isto não significa, entretanto, que estamos identificando os conjuntos-objeto $\mathrm{C}$ com suas extensões virtuais $\overline{\mathrm{C}}$. Por exemplo, supondo que o conjunto $\mathbf{R}$ dos números reais é um conjunto-objeto de $\mathcal{U}$, escrever $\infty \bar{\in} \overline{\mathbf{R}}$ é o mesmo que escrever $\infty \in \overline{\mathbf{R}}$, mas não que $\infty \in \mathbf{R}$, ou que $\mathbf{R}=\overline{\mathbf{R}}$.

\subsection{Funções Virtuais}

Suponhamos agora que o universo $\mathcal{U}$ contenha funções como objetos, isto é, como elementos.

DEFiniÇÃo: Diremos que uma função é uma função-objeto quando ela pertencer a $\mathcal{U}$ e, além disso, seu domínio e contradomínio forem, ambos, conjuntos-objeto.

DEFInIÇÃo: Consideremos o predicado ternário $\mathcal{P} \subset \mathcal{U}^{3}$ que afirma que $f$ é uma funçãoobjeto entre dois conjuntos-objeto D e C:

$$
\mathcal{P}(f, \mathrm{D}, \mathrm{C}): \quad f: \mathrm{D} \rightarrow \mathrm{C} .
$$

A extensão virtual deste predicado define o conceito de função virtual entre conjuntos virtuais:

$$
\overline{\mathcal{P}}(\varphi, \Delta, \Gamma): \quad \varphi: \Delta \rightarrow \Gamma .
$$

Segundo esta definição, se

$$
\varphi=\left\langle f_{1}, f_{2}, f_{3}, \ldots\right\rangle, \quad \Delta=\left\langle\mathrm{D}_{1}, \mathrm{D}_{2}, \mathrm{D}_{3}, \ldots\right\rangle \quad \text { e } \quad \Gamma=\left\langle\mathrm{C}_{1}, \mathrm{C}_{2}, \mathrm{C}_{3}, \ldots\right\rangle
$$

então dizer que $\varphi$ é uma função virtual do conjunto virtual $\Delta$ no conjunto virtual $\Gamma$ :

$$
\varphi: \Delta \rightarrow \Gamma
$$

equivale a dizer que existe $k \in \mathbf{N}$ tal que, para todo $i>k, f_{i}$ é uma função-objeto do conjunto-objeto $\mathrm{D}_{i}$ no conjunto-objeto $\mathrm{C}_{i}$ :

$$
f_{i}: \mathrm{D}_{i} \rightarrow \mathrm{C}_{i}
$$

DeFiniçÃo: A avaliação de funções-objeto $f: \mathrm{D} \rightarrow \mathrm{C}$ em elementos $a \in \mathrm{D}$ de seu domínio pode ser considerada como um funcional binário $\mathcal{F}$ :

$$
\mathcal{F}(f, a)=f(a)
$$


A extensão virtual deste funcional define a avaliação virtual de funções virtuais $\varphi: \Delta \rightarrow \Gamma$ em elementos $\alpha \in \Delta$ de seu domínio virtual:

$$
\overline{\mathcal{F}}(\varphi, \alpha)=\varphi(\alpha) .
$$

Esta definição é tal que, se

$$
\varphi=\left\langle f_{1}, f_{2}, f_{3}, \ldots\right\rangle \quad \quad \quad \text { e } \quad \alpha=\left\langle a_{1}, a_{2}, a_{3}, \ldots\right\rangle
$$

então

$$
\varphi(\alpha)=\left\langle f_{1}\left(a_{1}\right), f_{2}\left(a_{2}\right), f_{3}\left(a_{3}\right), \ldots\right\rangle,
$$

onde os termos $f_{i}\left(a_{i}\right)$ estão bem definidos a partir de um certo valor do índice $i$. É claro, portanto, que $\varphi(\alpha) \in \Gamma$.

DEFINIÇÃo: Se $\varphi: \Delta \rightarrow \Gamma$ é uma função virtual, a avaliação virtual definida acima nos permite associar a cada $\xi \in[\Delta]$ um e um único $\varphi(\xi) \in[\Gamma]$. Representaremos por $[\varphi]$ esta aplicação do conjunto real $[\Delta] \subset \overline{\mathcal{U}}$ no conjunto real $[\Gamma] \subset \overline{\mathcal{U}}$.

Já identificamos, na seção anterior, $\Delta \operatorname{com}[\Delta]$ e $\Gamma$ com $[\Gamma]$. Gostaríamos agora de identificar $\varphi \operatorname{com}[\varphi]$, mas antes devemos verificar que:

Proposição: Se $\varphi$ e $\psi$ são funções virtuais então:

$$
\varphi=\psi \quad \text { se e somente se } \quad[\varphi]=[\psi] .
$$

Prova: É claro que se $\varphi=\psi$ então $[\varphi]=[\psi]$. Para provar a recíproca, suponhamos que $\varphi \neq \psi$, e que

$$
\varphi=\left\langle f_{1}, f_{2}, f_{3}, \ldots\right\rangle \quad \text { e } \quad \psi=\left\langle g_{1}, g_{2}, g_{3}, \ldots\right\rangle .
$$

Para todo $k \in \mathbf{N}$, existe $i>k$ tal que $f_{i} \neq g_{i}$. Ou seja, para todo $k \in \mathbf{N}$, existe $i>k$ tal que:

$$
f_{i} \text { e } g_{i} \text { não têm o mesmo domínio; }
$$

ou

$$
f_{i} \text { e } g_{i} \text { não têm o mesmo contradomínio; }
$$

$\mathrm{ou}$

$$
\text { existe } x \in \mathcal{U} \text { tal que } f_{i}(x) \neq g_{i}(x) \text {. }
$$

Portanto, pelo menos uma das três condições abaixo está satisfeita:

(i) para todo $k \in \mathbf{N}$, existe $i>k$ tal que $f_{i}$ e $g_{i}$ não têm o mesmo domínio;

(ii) para todo $k \in \mathbf{N}$, existe $i>k$ tal que $f_{i}$ e $g_{i}$ não têm o mesmo contradomínio;

(iii) para todo $k \in \mathbf{N}$, existem $i>k$ e $x_{i} \in \mathcal{U}$ tais que $f_{i}\left(x_{i}\right) \neq g_{i}\left(x_{i}\right)$.

Se (i) for verdadeira então $[\varphi]$ e $[\psi]$ não têm o mesmo domínio. Se vale (ii) então $[\varphi]$ e $[\psi]$ não têm o mesmo contradomínio. Finalmente, a condição (iii) implica a existência de um objeto virtual $\xi$ que pertence tanto ao domínio de $[\varphi]$ quanto ao de $[\psi]$, e para o qual $[\varphi](\xi) \neq[\psi](\xi)$. Em qualquer destes três casos teremos $[\varphi] \neq[\psi]$. 
O resultado acima nos mostra que podemos identificar, com segurança, cada função virtual $\varphi: \Delta \rightarrow \Gamma$ com a aplicação $[\varphi]:[\Delta] \rightarrow[\Gamma]$ correspondente, isto é:

$$
\varphi=[\varphi] \text {. }
$$

É importante observar, entretanto, que nem toda aplicação de um conjunto virtual em outro conjunto virtual é uma função virtual, (segundo a identificação acima). Ou seja, nem toda aplicação entre conjuntos virtuais pode ser escrito na forma $[\varphi], \operatorname{com} \varphi$ sendo uma função virtual. A título de contra-exemplo:

Proposição: Se $\mathrm{D}=\{a, b\}$ é um conjunto-objeto com dois elementos (distintos entre si) então a aplicação $\Phi: \overline{\mathrm{D}} \rightarrow \overline{\mathrm{D}}$ definida por:

$$
\Phi(\xi)= \begin{cases}\bar{a} & , \text { se } \xi=\bar{a} \text { ou } \xi=\bar{b} \\ \bar{b} & , \text { caso contrário. }\end{cases}
$$

não é uma função virtual.

Prova: Suponhamos, a fim de obter uma contradição, que $\Phi=[\varphi]$, onde $\varphi=\left\langle f_{1}, f_{2}, \ldots\right\rangle$ é uma função virtual. Como $\Phi(\bar{a})=\bar{a}$, existe $k_{1} \in \mathbf{N}$ tal que, para todo $i>k_{1}$, $f_{i}(a)=a$. Por outro lado, como $\Phi(\bar{b})=\bar{a}$, existe $k_{2} \in \mathbf{N}$ tal que, para todo $i>k_{2}$, $f_{i}(b)=a$.

Tomando agora $\gamma=\langle a, b, a, b, a, b, \ldots\rangle \in \overline{\mathrm{D}}$, concluímos que $\varphi(\gamma)=\bar{a}$, pois existe $k=\operatorname{máx}\left\{k_{1}, k_{2}\right\}$ tal que $f_{i}(a)=f_{i}(b)=a$ para todo $i>k$. Mas, pela definição de $\Phi$, deveríamos ter $\varphi(\gamma)=\bar{b}$, pois $\gamma \neq \bar{a}$ e $\gamma \neq \bar{b}$.

Tendo feito a identificação acima, passaremos a considerar as funções virtuais como um tipo especial de aplicação entre conjuntos virtuais. Consequentemente, negligenciaremos, daqui por diante, distinção entre avaliação virtual e avaliação real.

\subsection{Virtualização de Parâmetros}

A partir deste ponto, estaremos supondo que:

(i) todos os subconjuntos de $\mathbf{R}$ pertencem a $\mathcal{U}$; e

(ii) todas as funções definidas de subconjuntos de $\mathbf{R}$ a valores em subconjuntos de $\mathbf{R}$ pertencem a $\mathcal{U}$.

Deste modo, todos os subconjuntos não-vazios de $\mathbf{R}$ são conjuntos-objeto, e todas as funções que levam números reais em números reais são funções-objeto.

DEFINIÇÃO: $\operatorname{Um}$ par $(\mathcal{P}, \mathcal{Q})$ descreve uma família parametrizada de conjuntos-objeto quando $\mathcal{P}$ é um predicado binário, $\mathcal{Q}$ um atributo, e a coleção:

$$
\{x \in \mathcal{U} \mid \mathcal{P}(x, a)\}
$$

é um conjunto-objeto para todo $a$ tal que $\mathcal{Q}(a)$. Neste caso, chamaremos o objeto $a \in \mathcal{U}$ de parâmetro da família de conjuntos-objeto. 
Assim, um par $(\mathcal{P}, \mathcal{Q})$ descreve uma família parametrizada de conjuntos-objeto quando a aplicação $\mathcal{G}$ dada por:

$$
\mathcal{G}(a)=\{x \in \mathcal{U} \mid \mathcal{P}(x, a)\}
$$

for um funcional unário com domínio $\mathcal{Q}$. Por exemplo, o conjunto:

$$
\mathbf{R}_{a}=\left\{x \in \mathbf{R} \mid x^{2}<a\right\}
$$

é um conjunto-objeto para todo $a>0$. Portanto os conjuntos $\mathbf{R}_{a}$, com $a>0$, formam uma família parametrizada de conjuntos-objeto, descrita formalmente pelo par $(\mathcal{P}, \mathcal{Q})$ dado por:

$$
\begin{aligned}
& \mathcal{P}(x, a): \quad x^{2}<a \\
& \mathcal{Q}(a): \quad a>0 .
\end{aligned}
$$

Neste caso, o funcional unário $\mathcal{G}: \mathcal{Q} \rightarrow \mathcal{U}$ mencionado acima é dado por:

$$
\mathcal{G}(a)=\mathbf{R}_{a} .
$$

DefiniÇÃo: Seja $(\mathcal{P}, \mathcal{Q})$ uma família parametrizada de conjuntos-objeto. Diremos que a coleção de objetos virtuais $\Gamma_{\alpha} \subset \overline{\mathcal{U}}$ dada por:

$$
\Gamma_{\alpha}=\{\xi \in \overline{\mathcal{U}} \mid \overline{\mathcal{P}}(\xi, \alpha)\}
$$

foi obtida por virtualização do parâmetro desta família se valer $\overline{\mathcal{Q}}(\alpha)$.

Retomando o exemplo anterior, diremos que a coleção de números virtuais:

$$
\Gamma_{\alpha}=\left\{\xi \in \overline{\mathbf{R}} \mid \xi^{2}<\alpha\right\}
$$

foi obtida por virtualização do parâmetro da família $\mathbf{R}_{a}$ se valer $\alpha>0$.

ProposiçÃo: Se uma coleção de objetos virtuais foi obtida por virtualização do parâmetro de uma família de conjuntos-objeto então ela é um conjunto virtual.

Prova: Se $(\mathcal{P}, \mathcal{Q})$ descreve a família parametrizada de conjuntos-objeto, então aplicação dada por:

$$
\mathcal{G}(a)=\{x \in \mathcal{U} \mid \mathcal{P}(x, a)\}
$$

é um funcional unário $\mathcal{G}: \mathcal{Q} \rightarrow \mathcal{U}$. Basta então aplicar o TEV à afirmação:

Para todo $a \in \mathcal{U}$ tal que $\mathcal{Q}(a)$, temos que $\mathcal{G}(a)$ é um conjunto-objeto. 
O resultado acima nos fornece uma condição suficiente para concluirmos que uma dada coleção de objetos virtuais é um conjunto virtual. Voltando ao da família $\mathbf{R}_{a}$, concluímos que, para todo $\alpha>0$, a coleção de números virtuais:

$$
\Gamma_{\alpha}=\left\{\xi \in \overline{\mathbf{R}} \mid \xi^{2}<\alpha\right\}
$$

é um conjunto virtual. Em particular, a coleção:

$$
\Gamma_{\infty}=\left\{\xi \in \overline{\mathbf{R}} \mid \xi^{2}<\infty\right\}
$$

é um conjunto virtual.

Analogamente, a proposição acima nos garante que as coleções seguintes de números virtuais são todas conjuntos virtuais:

$$
\begin{gathered}
\{\xi \in \overline{\mathbf{R}} \mid \xi \nprec \infty\} \\
\{\xi \in \overline{\mathbf{R}} \mid \xi<\infty \text { e } \xi \nprec 0\} . \\
\{\xi \in \overline{\mathbf{R}} \mid \xi \text { é uma mistura de } 0 \text { e } \infty\}
\end{gathered}
$$

Entretanto, estes outros subconjuntos de $\overline{\mathbf{R}}$ não são conjuntos virtuais:

$$
\begin{gathered}
\{\xi \in \overline{\mathbf{R}} \mid \xi \neq 0\} \\
\{\xi \in \overline{\mathbf{R}} \mid \xi \asymp 1\} \\
\{\xi \in \overline{\mathbf{R}} \mid \xi>1 \text { ou } \xi=0\} .
\end{gathered}
$$

É importante observar que o objeto que parametriza uma família de conjuntos não precisa ser do mesmo tipo que os elementos destes conjuntos. Por exemplo, podemos considerar os intervalos fechados:

$$
[a, b]=\{x \in \mathbf{R} \mid a \leq x \leq b\}
$$

como uma família parametrizada de conjuntos-objeto, onde o parâmetro é o par de extremos $(a, b)$, que esta sujeito à restrição $a \leq b$. Assim, pelo proposição anterior, os intervalos fechados virtuais:

$$
[\alpha, \beta]=\{\xi \in \overline{\mathbf{R}} \mid \alpha \leq \xi \leq \beta\}
$$

são conjuntos virtuais sempre que $\alpha \leq \beta$. Não é difícil ver que se $\alpha=\left\langle a_{1}, a_{2}, a_{3}, \ldots\right\rangle$ e $\beta=\left\langle b_{1}, b_{2}, b_{3}, \ldots\right\rangle$, então:

$$
[\alpha, \beta]=\left\langle\left[a_{1}, b_{1}\right],\left[a_{2}, b_{2}\right],\left[a_{3}, b_{3}\right], \ldots\right\rangle
$$


onde os intervalos $\left[a_{i}, b_{i}\right]$ estão bem definidos a partir de um certo valor do índice $i$. Em particular, o intervalo virtual $[-\infty, \infty] \subset \overline{\mathbf{R}}$ é a classe de equivalência:

$$
[\infty, \infty]=\langle[1,1],[2,2],[3,3], \ldots\rangle
$$

Devemos notar que $[-\infty, \infty] \neq \overline{\mathbf{R}}$. A título de contra-exemplo: $2 \infty \notin[-\infty, \infty]$.

DEFINIÇÃo: Um par $(\mathcal{F}, \mathcal{Q})$ descreve uma família parametrizada de funções-objeto quando $\mathcal{F}$ é um funcional binário, $\mathcal{Q}$ um atributo, e a aplicação:

$$
f(x)=\mathcal{F}(x, a)
$$

é uma função-objeto para todo $a$ tal que $\mathcal{Q}(a)$. Neste caso, chamaremos o objeto $a \in \mathcal{U}$ de parâmetro da família de funções-objeto.

Ou seja, um par $(\mathcal{F}, \mathcal{Q})$ descreve uma família parametrizada de conjuntos-objeto quando a aplicação $\mathcal{G}$ dada por:

$$
\mathcal{G}(a)=f_{a}, \quad \text { com } \quad f_{a}(x)=\mathcal{F}(x, a)
$$

for um funcional unário com domínio $\mathcal{Q}$. Exemplificando: estamos supondo que todos as funções entre números reais pertencem a $\mathcal{U}$, logo, para qualquer $a \geq 0$, a aplicação:

$$
f_{a}(x)=\sqrt{a^{2}-x^{2}}
$$

é uma função-objeto definida do intervalo $[-a, a]$ a valores em $\mathbf{R}_{+}$. Portanto, as funções $f_{a}$ formam uma família parametrizada de funções-objeto, onde o parâmetro $a \in \mathbf{R}$ está sujeito à restrição $a \geq 0$. Formalmente, esta família está descrita pelo par $(\mathcal{F}, \mathcal{Q})$ dado por:

$$
\begin{gathered}
\mathcal{F}(x, a)=f_{a}(x)=\sqrt{a^{2}-x^{2}}, \\
\mathcal{Q}(a): \quad a \geq 0 .
\end{gathered}
$$

Neste caso, o funcional unário $\mathcal{G}: \mathcal{Q} \rightarrow \mathcal{U}$ mencionado acima é dado por:

$$
\mathcal{G}(a)=f_{a}
$$

DefiniçÃo: Seja $(\mathcal{F}, \mathcal{Q})$ uma família parametrizada de funções-objeto. Diremos que a aplicação $\varphi_{\alpha}$ dada por:

$$
\varphi(\xi)=\overline{\mathcal{F}}(\xi, \alpha)
$$

foi obtida por virtualização do parâmetro desta família se valer $\overline{\mathcal{Q}}(\alpha)$. 
Retomando o exemplo acima, diremos que a aplicação:

$$
\varphi_{\alpha}(\xi)=\sqrt{\alpha^{2}-\xi^{2}}
$$

foi obtida por virtualização do parâmetro da família $f_{a}$ se valer $\alpha \geq 0$.

ProposiçÃo: Se uma aplicação entre objetos virtuais foi obtida por virtualização do parâmetro de uma família de funções-objeto então ela é uma função virtual.

Prova: Se $(\mathcal{F}, \mathcal{Q})$ descreve a família parametrizada de funções-objeto, então aplicação dada por:

$$
\mathcal{G}(a)=f_{a}, \quad \text { com } \quad f_{a}(x)=\mathcal{F}(x, a),
$$

é um funcional unário $\mathcal{G}: \mathcal{Q} \rightarrow \mathcal{U}$. Basta então aplicar o TEV à afirmação:

Para todo $a \in \mathcal{U}$ tal que $\mathcal{Q}(a)$, temos que $\mathcal{G}(a)$ é uma função-objeto.

Voltando mais uma vez ao exemplo da família $f_{a}$, concluímos que, para qualquer $\alpha \geq 0$, a aplicação:

$$
\begin{aligned}
\varphi_{\alpha}:[-\alpha, \alpha] \rightarrow \overline{\mathbf{R}_{+}} \\
\xi \mapsto \varphi_{\alpha}(\xi)=\sqrt{\alpha^{2}-\xi^{2}} .
\end{aligned}
$$

é uma função virtual. Em particular, para $\alpha=\infty$, a aplicação:

$$
\begin{aligned}
\varphi_{\infty}: & {[-\infty, \infty] \rightarrow \overline{\mathbf{R}_{+}} } \\
\xi & \mapsto \varphi_{\infty}(\xi)=\sqrt{\infty^{2}-\xi^{2}}
\end{aligned}
$$

é uma função virtual, que pode ser escrita como:

$$
\varphi_{\infty}=\left\langle f_{1}, f_{2}, f_{3}, \ldots\right\rangle \in \overline{\mathcal{U}}, \quad \text { onde } \quad f_{a}(x)=\sqrt{a^{2}-x^{2}} .
$$

O resultado acima nos fornece um meio para construir facilmente uma grande quantidade de funções virtuais: basta virtualizar o parâmetro de qualquer família de funçõesobjeto. Por exemplo, as aplicações seguintes são todas funções virtuais:

$$
\begin{gathered}
\log _{\infty}: \overline{\mathbf{R}_{+}^{*}} \rightarrow \overline{\mathbf{R}} \\
\xi \mapsto \log _{\infty} \xi \\
\varphi: \overline{\mathbf{R}} \rightarrow \overline{\mathbf{R}} \\
\xi \mapsto \varphi(\xi)=\xi^{\infty}=\underbrace{\xi \cdot \xi \cdot \xi \ldots \xi}_{\infty \text { vezes }}
\end{gathered}
$$




$$
\begin{aligned}
\psi: \overline{\mathbf{R}} & \rightarrow \overline{\mathbf{R}_{+}} \\
\xi & \mapsto \psi(\xi)=\frac{\infty}{1+\infty^{2} \xi^{2}} .
\end{aligned}
$$

Podemos também definir explicitamente uma família parametrizada de funções:

$$
f_{a}(x)=\left\{\begin{array}{lll}
a & , \text { se } & |x|<(1 / 2 a) \\
0 & , \text { se } & |x| \geq(1 / 2 a)
\end{array}\right.
$$

e então virtualizar seu parâmetro:

$$
\begin{aligned}
\varphi_{\alpha}: & \overline{\mathbf{R}} \rightarrow \overline{\mathbf{R}_{+}} \\
\xi & \mapsto \varphi_{\alpha}(\xi)=\overline{\mathcal{F}}(\xi, \alpha),
\end{aligned}
$$

onde $\mathcal{F}(x, a)=f_{a}(x)$. Fazendo, por exemplo, $\alpha=\infty$, obtemos:

$$
\begin{aligned}
\chi: \overline{\mathbf{R}} & \rightarrow \overline{\mathbf{R}_{+}} \\
\xi & \mapsto \chi(\xi)=\overline{\mathcal{F}}(\xi, \infty) .
\end{aligned}
$$

Esta função virtual $\chi$ é tal que:

$$
\chi(\xi)=\left\{\begin{array}{lll}
\infty & , \text { se } & |\xi|<1 / 2 \infty \\
0 & , \text { se } & |\xi| \geq 1 / 2 \infty
\end{array}\right.
$$

Este par de cláusulas, entretanto, não é suficiente para definir $\chi(\xi)$ para todo $\xi \in \overline{\mathbf{R}}$, pois há números virtuais $\xi$ que não se enquadram em nenhuma das duas condições acima.

É importante observar que o objeto que parametriza uma família de funções não precisa ser do mesmo tipo que a variável independente destas funções. Por exemplo, podemos considerar os polinmios em uma variável real como uma família parametrizada pela upla dos coeficientes:

$$
f_{\left(a_{1}, a_{2}, \ldots, a_{n}\right)}(x)=a_{1} x^{n-1}+a_{2} x^{n-2}+\cdots+a_{n} .
$$

A virtualização do parâmetro desta família define os polinmios virtuais em uma variável:

$$
\varphi_{\left(\alpha_{1}, \alpha_{2}, \ldots, \alpha_{\nu}\right)}(\xi)=\alpha_{1} \xi^{\nu-1}+\alpha_{2} \xi^{\nu-2}+\cdots+\alpha_{\nu}
$$

para qualquer $\nu$-upla $\left(\alpha_{1}, \alpha_{2}, \ldots, \alpha_{\nu}\right)$ de números virtuais. Segundo a proposição acima, estes polinmios virtuais são todos funções virtuais. 


\subsection{Continuidade Virtual}

Vamos agora estender a noção de continuidade às funções numéricas virtuais, isto é, às funções virtuais que levam números virtuais em números virtuais:

DefiniçÃo: A continuidade de uma função real $f: \mathrm{D} \rightarrow \mathrm{C}$ em um ponto $x \in \mathrm{D}$ define um predicado binário $\mathcal{P} \subset \mathcal{U}^{2}$ :

$$
\mathcal{P}(f, x): \quad f \text { é contínua em } x .
$$

A extensão virtual deste predicado:

$$
\overline{\mathcal{P}}(\varphi, \xi): \quad \varphi \text { é contínua em } \xi .
$$

define a continuidade de uma função virtual $\varphi: \Delta \rightarrow \overline{\mathbf{R}}$ em um ponto $\xi \in \Delta$.

Segundo esta definição, se

$$
\varphi=\left\langle f_{1}, f_{2}, f_{3}, \ldots\right\rangle \quad \text { e } \quad \xi=\left\langle x_{1}, x_{2}, x_{3}, \ldots\right\rangle
$$

então $\varphi$ é contínua em $\xi$ quando existe $n \in \mathbf{N}$ tal que $f_{i}$ é contínua em $x_{i}$, para todo $i>n$.

Podemos também considerar a continuidade de uma função real em todos os pontos do seu domínio:

DEFINIÇÃO: Seja $\mathcal{P} \subset \mathcal{U}$ o atributo:

$$
\mathcal{P}(f): \quad f \text { é contínua. }
$$

A extensão virtual deste predicado define a continuidade de uma função virtual:

$$
\overline{\mathcal{P}}(\varphi): \quad \varphi \text { é contínua. }
$$

Segundo esta definição, uma função virtual $\varphi=\left\langle f_{1}, f_{2}, f_{3}, \ldots\right\rangle$ é contínua quando existe $n \in \mathbf{N}$ tal que as funções reais $f_{i}$ são contínuas sempre que $i>n$.

Proposição: Uma função virtual é contínua se e somente se ela for contínua em todos os pontos do seu domínio.

Prova: Para verificar que uma função virtual contínua é contínua em todos os pontos do seu domínio basta aplicar o TEV à afirmação:

Para todo par $(f, x)$ tal que $f$ é contínua e $x$ pertence ao seu domínio,

a função $f$ é contínua em $x$.

Para demonstrar a recíproca, suponhamos que $\varphi=\left\langle f_{1}, f_{2}, \ldots\right\rangle$ não seja contínua em $\xi=\left\langle x_{1}, x_{2}, \ldots\right\rangle$. Assim, para todo $k \in \mathbf{N}$, existe $i>k$ tal que $f_{i}$ é descontínua em $x_{i}$. Como o domínio $\Delta$ de $\varphi$ é um conjunto-objeto, temos $\Delta \nsucc \varnothing$. Concluímos então que existe $\xi \in \Delta$ tal que $\varphi$ é descontínua em $\xi$. 
Podemos utilizar o TEV para verificar a continuidade de funções virtuais obtidas por virtualização do parâmetro de uma família de funções-objeto. Por exemplo, aplicando o TEV à afirmação:

$$
\begin{aligned}
& \text { Para todo } a \in \mathbf{R} \text { tal que } 0<a \neq 1 \text {, } \\
& \text { a função } \log _{a} \text { é contínua, }
\end{aligned}
$$

concluímos que:

$$
\text { Para todo } \alpha \in \overline{\mathbf{R}} \text { tal que } 0<\alpha \nprec 1 \text {, }
$$

a função $\log _{\alpha}$ é contínua.

Em particular, a função virtual $\log _{\infty}$ é contínua.

Analogamente, obtemos que a função virtual $\psi: \overline{\mathbf{R}} \rightarrow \overline{\mathbf{R}_{+}}$definida no final da seção anterior:

$$
\psi(\xi)=\frac{\infty}{1+\infty^{2} \xi^{2}}
$$

também é contínua, em todo $\xi \in \overline{\mathbf{R}}$, inclusive para valores reais $x \in \mathbf{R}$ do seu argumento, nos quais:

$$
\psi(x) \approx\left\{\begin{array}{lll}
\infty & , \text { se } & x=0 \\
0 & , \text { se } & x \neq 0
\end{array}\right.
$$

Vimos também no final da seção anterior a família:

$$
f_{a}(x)=\left\{\begin{array}{lll}
a & , \text { se } & |x|<(1 / 2 a) \\
0 & , \text { se } & |x| \geq(1 / 2 a) .
\end{array}\right.
$$

Neste caso, temos que:

Para todo par $(x, a) \in \mathbf{R}^{2}$ tal que $x \nprec(1 / 2 a)$ e $a>0$,

a função $f_{a}$ é contínua em $x$.

Portanto o TEV nos garante que:

Para todo par $(\xi, \alpha) \in \overline{\mathbf{R}}^{2}$ tal que $\xi \neq(1 / 2 \alpha)$ e $\alpha>0$,

a função $\varphi_{\alpha}$ é contínua em $\xi$.

Em particular, para $\alpha=\infty$, concluímos que a função $\chi=\varphi_{\infty}$ é contínua em todo $\xi \nprec(1 / 2 \infty)$, inclusive nos valores reais $x \in \mathbf{R}$ do seu argumento, para os quais:

$$
\chi(x) \approx\left\{\begin{array}{lll}
\infty & , \text { se } & x=0 \\
0 & , \text { se } & x \neq 0
\end{array}\right.
$$

A continuidade de uma função real $f$ em um ponto $x$ do seu domínio foi definida, no capítulo anterior, pela condição:

$$
\alpha \approx x \quad \text { implica } \quad f(\alpha) \approx f(x)
$$


É importante observar que a continuidade virtual definida acima não exige que esta condição esteja satisfeita pelas funções virtuais. Por exemplo, a função virtual $\psi$ acima é contínua em todo $\overline{\mathbf{R}}$, mas calculando esta função no infinitésimo $\alpha=1 / \sqrt{\infty}$ obtemos:

$$
\psi(1 / \sqrt{\infty})=\frac{\infty}{1+\infty^{2} \frac{1}{\infty}}=\frac{\infty}{1+\infty} \approx 1
$$

Ou seja:

$$
1 / \sqrt{\infty} \approx 0 \quad \text { mas } \quad \psi(1 / \sqrt{\infty}) \not \approx \psi(0) .
$$

De forma análoga, definindo a continuidade uniforme de uma função virtual $\varphi$ pela extensão do atributo "ser uniformemente contínua", não teremos necessariamente que:

$$
\alpha \approx \beta \quad \text { implica } \quad \varphi(\alpha) \approx \varphi(\beta)
$$

Isto pode ser verificado observando que a função $\psi$ acima é uniformemente contínua em $\overline{\mathbf{R}}$.

\subsection{Derivação Virtual}

O objetivo desta seção é definir a derivada de funções numéricas virtuais, e mostrar que o algoritmo de derivação usual do Cálculo se aplica a esta derivação virtual:

DEFINIÇÃo: A derivabilidade de uma função real $f: \mathrm{D} \rightarrow \mathrm{C}$ em um ponto $x \in \mathrm{D}$ define um predicado binário $\mathcal{P} \subset \mathcal{U}^{2}$ :

$$
\mathcal{P}(f, x): \quad f \text { é derivável em } x
$$

A extensão virtual deste predicado:

$$
\overline{\mathcal{P}}(\varphi, \xi): \quad \varphi \text { é derivável em } \xi
$$

define a derivabilidade de uma função virtual $\varphi: \Delta \rightarrow \overline{\mathbf{R}}$ em um ponto $\xi \in \Delta$.

Segundo essa definição, se

$$
\varphi=\left\langle f_{1}, f_{2}, f_{3}, \ldots\right\rangle \quad \quad \quad \text { e } \quad\left\langle x_{1}, x_{2}, x_{3}, \ldots\right\rangle
$$

então $\varphi$ é derivável em $\xi$ quando existe $n \in \mathbf{N}$ tal que $f_{i}$ é derivável em $x_{i}$, para todo $i>n$.

Podemos também considerar a derivabilidade de uma função real em todos os pontos do seu domínio: 
DEFINIÇÃo: Seja $\mathcal{P} \subset \mathcal{U}$ o atributo:

$$
\mathcal{P}(f): \quad f \text { é derivável. }
$$

A extensão virtual deste predicado define a derivabilidade de uma função virtual:

$$
\overline{\mathcal{P}}(\varphi): \quad \varphi \text { é derivável. }
$$

Segundo essa definição, uma função virtual $\varphi=\left\langle f_{1}, f_{2}, f_{3}, \ldots\right\rangle$ é derivável quando existe $n \in \mathbf{N}$ tal que as funções reais $f_{i}$ são deriváveis sempre que $i>n$.

A demonstração do resultado seguinte é análoga à da proposição correspondente para a continuidade, apresentada na seção anterior:

Proposição: Uma função virtual é derivável se e somente se ela for derivável em todos os pontos do seu domínio.

DEFINIÇÃo: Diremos que uma função real $f$ tem derivada quando ela for derivável em pelo menos um ponto do seu domínio, e neste caso representaremos sua derivada por $f^{\prime}$. Assim, o domínio de $f^{\prime}$ é um subconjunto não vazio do domínio de $f$, eventualmente um subconjunto próprio.

DEFINIÇÃO: Seja $\mathcal{P} \subset \mathcal{U}$ o atributo dado por:

$$
\mathcal{P}(f): \quad f \text { tem derivada. }
$$

A extensão virtual deste atributo:

$$
\overline{\mathcal{P}}(\varphi): \quad \varphi \text { tem derivada. }
$$

define quando uma função virtual $\varphi$ tem derivada.

Segundo essa definição, uma função virtual $\varphi=\left\langle f_{1}, f_{2}, f_{3}, \ldots\right\rangle$ tem derivada quando existe $n \in \mathbf{N}$ tal que as funções reais $f_{i}$ têm derivada sempre que $i>n$.

Proposição: Uma função virtual tem derivada se e somente se ela for derivável em pelo menos um ponto do seu domínio.

Prova: Basta aplicar o TEV às afirmações:

Para toda função real $f$ que tem derivada, existe $x$ tal que $f$ é derivável em $x$.

e

Para toda função real $f$ tal que existe $x \operatorname{com} f$ derivável em $x$, $f$ é derivável. 
DefiniçÃo: Seja $\mathcal{F}$ o funcional unário que deriva as funções reais que têm derivada:

$$
\mathcal{F}(f)=f^{\prime}
$$

A extensão virtual deste funcional define a derivada das funções virtuais (que têm derivada):

$$
\overline{\mathcal{F}}(\varphi)=\varphi^{\prime}
$$

Por esta definição, a derivada de uma função virtual $\varphi=\left\langle f_{1}, f_{2}, f_{3}, \ldots\right\rangle$ é a classe de equivalência:

$$
\varphi^{\prime}=\left\langle f_{1}^{\prime}, f_{2}^{\prime}, f_{3}^{\prime}, \ldots\right\rangle
$$

onde as derivadas $f_{i}^{\prime}$ podem se calculadas a partir de um certo valor do índice $i$.

O TEV nos garante que, se $\varphi$ é uma função virtual que tem derivada, então o domínio de $\varphi^{\prime}$ é um subconjunto (virtual) não-vazio do domínio de $\varphi$, eventualmente um subconjunto próprio. Podemos também utilizar o TEV para calcular a derivada de funções virtuais obtidas por virtualização do parâmetro de uma família de funções-objeto. Por exemplo, a derivada da função $f(x)=x^{n}$ é dada por $f^{\prime}(x)=n x^{n-1}$, para qualquer $n \in \mathbf{N}$. Portanto, pelo TEV, a derivada da função virtual:

$$
\begin{aligned}
\varphi: & \mathbf{\mathbf { R }} \rightarrow \overline{\mathbf{R}} \\
\xi & \mapsto \varphi(\xi)=\xi^{\nu}
\end{aligned}
$$

é a função virtual:

$$
\begin{aligned}
\varphi^{\prime}: \overline{\mathbf{R}} & \rightarrow \overline{\mathbf{R}} \\
\xi & \mapsto \varphi(\xi)=\nu \xi^{\nu-1},
\end{aligned}
$$

para qualquer $\nu \in \overline{\mathbf{N}}$. Em particular, para $\nu=\infty$, temos:

$$
\frac{d}{d \xi}\left(\xi^{\infty}\right)=\infty \xi^{\infty-1}
$$

As regras de derivação são afirmações sobre o processo de derivação, considerado como um funcional que leva funções-objeto em funções-objeto. Com as definições acima, o TEV nos garante que todas elas valem também para funções virtuais. Por exemplo:

$$
\begin{gathered}
(\varphi+\psi)^{\prime}=\varphi^{\prime}+\psi^{\prime} \\
(\varphi \psi)^{\prime}=\varphi^{\prime} \psi+\varphi \psi^{\prime} \\
(\varphi \circ \psi)^{\prime}=\left(\varphi^{\prime} \circ \psi\right) \psi^{\prime} .
\end{gathered}
$$

Portanto, o algoritmo de derivação usual do Cálculo pode ser utilizado para funções virtuais. 
Por exemplo, se:

$$
v=\frac{\infty}{1+\infty^{2} \xi^{2}}
$$

então:

$$
\frac{d v}{d \xi}=\frac{-\infty}{\left(1+\infty^{2} \xi^{2}\right)^{2}}\left(2 \infty^{2} \xi\right)=\frac{-2 \infty^{3} \xi}{\left(1+\infty^{2} \xi^{2}\right)^{2}} .
$$

A derivada de uma função real $f$ em um ponto $x$ do seu domínio foi definida na seç. 2.5 como sendo o número real $f^{\prime}(x)$ que satisfaz a condição:

$$
\alpha \sim x \quad \text { implica } \quad f^{\prime}(x) \approx \frac{f(\alpha)-f(x)}{\alpha-x} .
$$

Entretanto, a derivada de uma função virtual em um ponto do seu domínio, como definida acima, não satisfaz necessariamente esta condição. Por exemplo, calculando a derivada da função $v=\psi(\xi)$ acima em $\xi=0$ obtemos:

$$
\psi^{\prime}(\xi)=\left.\frac{d v}{d \xi}\right|_{\xi=0}=\frac{-2 \infty^{3} 0}{\left(1+\infty^{2} 0^{2}\right)^{2}}=0,
$$

(o que era de se esperar, pois $\varphi$ é uma função par) enquanto, para $x=0$ e $\alpha=(1 / \infty)$, temos:

$$
\begin{aligned}
\frac{\varphi(\alpha)-\varphi(x)}{\alpha-x} & =\frac{\frac{\infty}{1+\infty^{2}\left(\frac{1}{\infty}\right)^{2}}-\frac{\infty}{1+\infty^{2} 0^{2}}}{\frac{1}{\infty}-0} \\
& =\infty\left(\frac{\infty}{1+1}-\frac{\infty}{1+0}\right) \\
& =\infty\left(\frac{\infty}{2}-\infty\right) \\
& =-\frac{\infty^{2}}{2}
\end{aligned}
$$

Ou seja:

$$
(1 / \infty) \sim 0 \quad \text { mas } \quad \psi^{\prime}(0) \not \frac{\psi\left(\frac{1}{\infty}\right)-\psi(0)}{\frac{1}{\infty}-0} .
$$

\subsection{Integração Virtual}

Vamos agora definir a integração de funções virtuais. A definição de integral apresentada na seç. 2.7 (que, como vimos, equivale àquela devida a Riemann) será mais do que suficiente para os nossos propósitos. Assim, no que segue, integrabilidade e integrais de funções reais entre dois números reais devem ser entendidas neste sentido.

Na definição seguinte não supomos que $a \leq b$ : 
DeFiniÇÃo: Diremos que um número real $x$ está entre $a$ e $b$ quando:

$$
a \leq x \leq b \quad \text { ou } \quad b \leq x \leq a .
$$

Esta relação define um predicado ternário $\mathcal{P} \subset \mathcal{U}^{3}$ :

$$
\mathcal{P}(x, a, b): \quad x \text { está entre } a \text { e } b,
$$

cuja extensão virtual define a relação "estar entre" para números virtuais:

$$
\overline{\mathcal{P}}(\xi, \alpha, \beta): \quad \xi \text { está entre } \alpha \text { e } \beta \text {. }
$$

ProposiçÃo: Para quaisquer números virtuais $\alpha$ e $\beta$, a coleção de todos os virtuais que estão entre eles é um conjunto virtual.

Prova: Basta considerar a família parametrizada de conjuntos-objeto:

$$
\mathrm{C}_{(a, b)}=\{x \in \mathbf{R} \mid x \text { está entre } a \text { e } b\}
$$

e então virtualizar o parâmetro $(a, b)$.

Assim, diremos que se uma função virtual está definida entre dois números virtuais quando ela estiver definida em todo número virtual entre eles. Analogamente, diremos que se uma função virtual é contínua entre dois virtuais quando ela for contínua em todo virtual entre eles.

DEFINIÇÃo: A integrabilidade de uma função real $f$ entre dois números reais $a$ e $b$ define um predicado ternário $\mathcal{P} \subset \mathcal{U}^{3}$ :

$$
\mathcal{P}(f, a, b): \quad f \text { é integrável entre } a \text { e } b .
$$

A extensão virtual deste predicado define a integrabilidade de uma função virtual entre dois números virtuais:

$$
\overline{\mathcal{P}}(\varphi, \alpha, \beta): \quad \varphi \text { é integrável entre } \alpha \text { e } \beta .
$$

Segundo esta definição, se $\varphi=\left\langle f_{i}\right\rangle, \alpha=\left\langle a_{i}\right\rangle$ e $\beta=\left\langle b_{i}\right\rangle$, então $\varphi$ é integrável entre $\alpha$ e $\beta$ quando existe $n \in \mathbf{N}$ tal que, para todo $i>n$, a função $f_{i}$ é integrável entre $a_{i}$ e $b_{i}$.

DEFINIÇÃo: Seja $\mathcal{F}$ o funcional ternário que calcula a integral de uma função integrável entre dois pontos:

$$
\mathcal{F}(f, a, b)=\int_{a}^{b} f(x) d x .
$$

A extensão virtual deste funcional define a integral de uma função virtual integrável entre dois números virtuais:

$$
\overline{\mathcal{F}}(\varphi, \alpha, \beta)=\int_{\alpha}^{\beta} \varphi(\xi) d \xi .
$$

Nesta notação, o uso de uma letra grega para representar a variável de integração indica que se trata de uma integral virtual. 
Segundo esta definição, se $\varphi=\left\langle f_{i}\right\rangle, \alpha=\left\langle a_{i}\right\rangle$ e $\beta=\left\langle b_{i}\right\rangle$, então:

$$
\int_{\alpha}^{\beta} \varphi(\xi) d \xi=\left\langle\int_{a_{1}}^{b_{1}} f_{1}(x) d x, \quad \int_{a_{2}}^{b_{2}} f_{2}(x) d x, \quad \int_{a_{3}}^{b_{3}} f_{3}(x) d x, \ldots\right\rangle
$$

onde as integrais podem se calculadas a partir de um certo valor do índice $i$.

Sabemos que se uma função real está definida e é contínua entre dois números reais então ela é integrável entre estes dois números. O TEV nos garante então que:

Proposição: Toda função virtual definida e contínua entre dois números virtuais quaisquer é integrável entre estes dois números virtuais.

Por exemplo, os símbolos:

$$
\int_{0}^{\frac{1}{\infty}} \frac{\infty}{1+\infty^{2} \xi^{2}} d \xi
$$

$\mathrm{e}$

$$
\int_{-\infty^{2}}^{\infty^{\xi}} e^{\frac{\tau}{\infty}} d \tau
$$

estão perfeitamente definidos.

Podemos inclusive definir uma função virtual por:

$$
\varphi(\xi)=\int_{-\infty^{2}}^{\infty} e^{\frac{\tau}{\infty}} d \tau
$$

Esta função é a classe $\left\langle f_{1}, f_{2}, \ldots\right\rangle$ dada por:

$$
f_{i}(x)=\int_{-i^{2}}^{i^{x}} e^{\frac{t}{i}} d t
$$

A fim de simplificar a terminologia, convencionamos:

DEFINIÇÃo: Diremos simplesmente que uma função virtual é integrável (sem mencionar os extremos) quanto ela for integrável entre quaisquer dois números virtuais do seu domínio.

Sabemos também que a soma e o produto de duas funções reais integráveis também são integráveis. O TEV nos garante então que a soma e o produto de duas funções virtuais integráveis também são integráveis.

Da mesma forma, podemos utilizar o TEV para demonstrar que as integrais virtuais satisfazem as propriedades esperadas de uma integral, como, por exemplo, a aditividade com respeito ao intervalo de integração:

$$
\int_{\alpha}^{\gamma} \varphi(\xi) d \xi=\int_{\alpha}^{\beta} \varphi(\xi) d \xi+\int_{\beta}^{\gamma} \varphi(\xi) d \xi
$$


Não vamos listar aqui todas estas propriedades, pois é mais cmodo usar o TEV quando elas forem necessárias.

DefiniÇÃo: Diremos que um função virtual $\psi$ é uma primitiva de $\varphi$ quando $\psi^{\prime}=\varphi$, e chamaremos a coleção de todas as primitivas de uma função virtual de integral indefinida dessa função (assim como no fazemos para funções reais).

O TEV nos garante que duas primitivas de uma função virtual continua $\varphi:[\alpha, \beta] \rightarrow$ $\overline{\mathbf{R}}$ diferem por uma constante (virtual) aditiva. Assim, representaremos a integral indefinida dessa função por:

$$
\int \varphi(\xi) d \xi=\psi(\xi)+\kappa
$$

onde $\psi:[\alpha, \beta] \rightarrow \overline{\mathbf{R}}$ é uma primitiva particular de $\varphi$ e $\kappa \in \overline{\mathbf{R}}$ uma constante arbitrária, ficando subentendido que estamos nos referindo à coleção das funções virtuais que podem ser escritas desta forma.

Com as definições e convenções acima, o TEV nos mostra que todas as técnicas de integração do Cálculo tradicional se estendem para funções virtuais, inclusive com as notações (igualmente tradicionais) associadas a elas, bastando para tanto trocarmos as letras latinas por gregas.

Por exemplo, para calcular

$$
\int \frac{\infty}{1+\infty^{2} \xi^{2}} d \xi
$$

fazemos a mudança de variáveis $\mu=\infty \xi$, logo $d \mu=\infty d \xi$, e portanto:

$$
\int \frac{\infty}{1+\infty^{2} \xi^{2}} d \xi=\int \frac{d \mu}{1+\mu^{2}}=\operatorname{arctg} \mu+\kappa=\operatorname{arctg}(\infty \xi)+\kappa
$$

Aplicando o TEV à primeira forma do Teorema Fundamental do Cálculo obtemos:

Proposição: Se uma função virtual $\varphi$ está definida e é contínua entre $\alpha$ e $\beta$, então, para todo $\xi$ entre $\alpha$ e $\beta$ :

$$
\frac{d}{d \xi} \int_{\alpha}^{\xi} \varphi(\tau) d \tau=\varphi(\xi)
$$

Em outras palavras, a função virtual definida por:

$$
\psi(\xi)=\int_{\alpha}^{\xi} f(\tau) d \tau
$$

é uma primitiva de $\varphi$. 
O TEV nos fornece também a segunda forma do Teorema Fundamental do Cálculo para funções virtuais:

Proposição: Se uma função virtual $\varphi$ está definida e é contínua entre $\alpha$ e $\beta$, e $\psi$ é uma primitiva de $\varphi$, então:

$$
\int_{\alpha}^{\beta} \varphi(\xi) d \xi=\psi(\beta)-\psi(\alpha)
$$

Alguns exemplos:

$$
\begin{gathered}
\frac{d}{d \xi} \int_{-\infty^{2}}^{\infty^{\xi}} e^{\frac{\tau}{\infty}} d \tau=e^{\frac{1}{\infty} \infty^{\xi}} \frac{d}{d \xi}\left(\infty^{\xi}\right)=e^{\infty^{\xi-1}} \infty^{\xi} \ln \infty \\
\int_{0}^{\frac{1}{\infty}} \frac{\infty}{1+\infty^{2} \xi^{2}} d \xi=[\operatorname{arctg}(\infty \xi)]_{0}^{\frac{1}{\infty}}=\operatorname{arctg}\left(\infty \frac{1}{\infty}\right)-\operatorname{arctg}(\infty 0)=\operatorname{arctg} 1-\operatorname{arctg} 0=\frac{\pi}{4} .
\end{gathered}
$$

DEFINIÇÃO: Diremos que uma integral virtual:

$$
\int_{\alpha}^{\beta} \varphi(\xi) d \xi
$$

é redutivel quando ela estiver próxima de algum número real. Neste caso, usaremos o símbolo:

$$
\int_{\alpha}^{\beta} \varphi(x) d x
$$

para representar este número real, que chamaremos de integral reduzida da função $\varphi$ entre $\alpha$ e $\beta$. Este símbolo não será utilizado quando uma integral virtual for irredutível, de forma que uma integral com variável de integração real (letra latina), quando existir, sempre será um número real.

Para qualquer integral redutível, temos que:

$$
\int_{\alpha}^{\beta} \varphi(x) d x \approx \int_{a}^{b} \varphi(\xi) d \xi
$$

mas, em geral, não é verdade que:

$$
\int_{\alpha}^{\beta} \varphi(x) d x=\int_{a}^{b} \varphi(\xi) d \xi
$$

Por exemplo:

$$
\int_{1}^{\infty} \frac{d \xi}{\xi^{2}}=\left[\frac{-1}{\xi}\right]_{1}^{\infty}=-\frac{1}{\infty}+1
$$


$\log O$

$$
\int_{1}^{\infty} \frac{d x}{x^{2}}=1
$$

e

$$
\int_{1}^{\infty} \frac{d x}{x^{2}} \neq \int_{1}^{\infty} \frac{d \xi}{\xi^{2}}
$$

Analogamente:

$$
\int_{\frac{1}{\infty}}^{1} \frac{d \xi}{\sqrt{\xi}}=[2 \sqrt{\xi}]_{\frac{1}{\infty}}^{1}=2-2 \sqrt{\frac{1}{\infty}}
$$

$\log O$

$$
\int_{\frac{1}{\infty}}^{1} \frac{d x}{\sqrt{x}}=2
$$

e, portanto:

$$
\int_{\frac{1}{\infty}}^{1} \frac{d x}{\sqrt{x}} \neq \int_{\frac{1}{\infty}}^{1} \frac{d \xi}{\sqrt{\xi}} .
$$




\section{Capítulo 4}

\section{O Cálculo Delta de Dirac}

Apresentaremos agora uma formalização do Cálculo Delta de Dirac segundo os conceitos introduzidos nos capítulos anteriores. Tal formalização é bastante simples e natural, pois sua definições e provas formais correspondem exatamente às concepções de Dirac, e os métodos matemáticos empregados refletem fielmente o que os físicos têm em mente ao utilizar o Cálculo Delta.

\subsection{Funções de Dirac}

Nesta primeira seção, caracterizaremos as funções virtuais que possuem as propriedades básicas assumidas por Dirac para sua "função delta", e mostraremos que existem muitas funções virtuais com estas propriedades:

Definição: Diremos que uma função virtual $\delta: \overline{\mathbf{R}} \rightarrow \overline{\mathbf{R}}$ é uma função de Dirac quando $\delta$ for não-negativa: $\delta(\xi) \geq 0$, para qualquer $\xi \in \overline{\mathbf{R}}$; e existir um infinitésimo positivo $\varepsilon>0$ tal que:

(i) $|\xi| \geq \varepsilon$ implica $\delta(\xi)=0$; e

(ii) $\delta$ é integrável entre $-\varepsilon$ e $\varepsilon$, com esta integral redutível à unidade:

$$
\int_{-\varepsilon}^{\varepsilon} \delta(x) d x=1 .
$$

Antes de prosseguir, convém verificarmos que existem funções virtuais satisfazendo as duas condições acima. Para tanto, consideremos a família das funções reais $g_{a}: \mathbf{R} \rightarrow \mathbf{R}$ dadas por:

$$
g_{a}(x)=\left\{\begin{array}{ll}
a & , \text { se }|x|<1 / 2 a \\
0 & , \text { se }|x| \geq 1 / 2 a
\end{array} \quad(a>0)\right.
$$


Virtualizando o parâmetro $a$ obtemos, para cada $\alpha>0$, uma função virtual não-negativa $\varphi_{\alpha}: \overline{\mathbf{R}} \rightarrow \overline{\mathbf{R}}$ tal que:

(i) $\varphi_{\alpha}(\xi)=0$, para todo $|\xi| \geq 1 / 2 \alpha$; e

(ii) $\varphi_{\alpha}$ é integrável entre $-\alpha$ e $\alpha$, e está normalizada no sentido forte:

$$
\int_{-\alpha}^{\alpha} \varphi_{\alpha}(\xi) d \xi=1
$$

Portanto, tomando $\alpha>\mathbf{R}$, a função virtual $\delta_{\sqcap}=\varphi_{\alpha}$ satisfaz, para $\varepsilon=1 / 2 \alpha$, as duas condições acima, sendo portanto uma função de Dirac. Em particular, tomando $\alpha=\infty$, obtemos:

$$
\delta_{\sqcap}=\varphi_{\infty}=\left\langle g_{1}, g_{2}, g_{3}, \ldots\right\rangle
$$

A função virtual $\varphi_{\alpha}$ acima não é contínua nos valores de $\xi$ tais que $|\xi| \asymp 1 / 2 \alpha$, mas podemos construir funções de Dirac contínuas e infinitamente deriváveis em todo $\xi \in \overline{\mathbf{R}}$ :

Sabemos que existe uma função real $f: \mathbf{R} \rightarrow \mathbf{R}$ com as seguintes características:

(a) $f$ é infinitamente derivável em todo $x \in \mathbf{R}$;

(b) $f(x)=0$ para todo $x$ tal que $|x| \geq 1$;

(c) $f$ é crescente entre -1 e 0 , onde assume o valor $f(0)=1$, decrescente entre 0 e 1 ; e

(d) $f$ está normalizada:

$$
\int_{-1}^{1} f(x) d x=1 .
$$

A partir de uma tal função $f$, podemos construir uma família de funções reais $f_{a}$ por:

$$
f_{a}(x)=a \cdot f(a . x) \quad(a>0)
$$

e então definir $\psi_{\alpha}: \overline{\mathbf{R}} \rightarrow \overline{\mathbf{R}}$ virtualizando seu parâmetro. Assim, para cada $\alpha>0$, temos:

(a) $\psi_{\alpha}$ é infinitamente derivável em todo $\xi \in \overline{\mathbf{R}}$;

(b) $\psi_{\alpha}(\xi)=0$ para todo $\xi$ tal que $|\xi| \geq 1 / \alpha$;

(c) $\psi_{\alpha}$ é crescente entre $-1 / \alpha$ e 0 , onde assume o valor $\psi_{\alpha}(0)=\alpha$, decrescente entre 0 e $1 / \alpha$; e

(d) $\psi_{\alpha}$ está normalizada no sentido forte:

$$
\int_{-1 / \alpha}^{1 / \alpha} \psi_{\alpha}(\xi) d \xi=1
$$


Portanto, para todo $\alpha>\mathbf{R}$, a função virtual $\psi_{\alpha}$ é uma função de Dirac contínua e infinitamente derivável em todo $\xi \in \overline{\mathbf{R}}$. Em particular, para $\alpha=\infty$, temos uma função de Dirac $\delta_{0}: \overline{\mathbf{R}} \rightarrow \overline{\mathbf{R}}$ contínua e infinitamente derivável em todo $\xi \in \overline{\mathbf{R}}$, inclusive nos valores reais $x \in \mathbf{R}$ do seu argumento, para os quais:

$$
\delta_{0}(x)= \begin{cases}\infty & , \text { se } x=0 \\ 0 & , \text { se } x \neq 0\end{cases}
$$

Para que uma função virtual $\delta: \mathbf{R} \rightarrow \mathbf{R}$ seja uma função de Dirac, não é necessário que $\delta(0)=\infty$, ou mesmo que $\delta(0) \approx \infty$. Mas a condição $\delta(x)=0$, para todo real $x \neq 0$, é uma consequência imediata da existência de um infinitésimo $\varepsilon$ tal que $|\xi|>\varepsilon$ implica $\delta(\xi)=0$. Por exemplo, a função virtual $\psi: \overline{\mathbf{R}} \rightarrow \overline{\mathbf{R}}$ dada por:

$$
\psi(\xi)=\frac{\infty}{1+\infty^{2} \xi^{2}}
$$

é tal que $\psi(x) \approx 0$ para todo real $x \neq 0$ :

$$
\psi(x)=\frac{1}{\frac{1}{\infty}+\infty x^{2}} \approx 0 .
$$

Mas $\psi(\xi) \neq 0$, para qualquer $\xi \in \overline{\mathbf{R}}$, e portanto $\psi$ não é uma função de Dirac.

Podemos construir funções de Dirac com propriedades adicionais. Por exemplo, se $\delta_{0}: \overline{\mathbf{R}} \rightarrow \overline{\mathbf{R}}$ é a função construída acima então a função virtual $\delta_{+}: \overline{\mathbf{R}} \rightarrow \overline{\mathbf{R}}$ dada por:

$$
\delta_{+}(\xi)=\delta_{0}\left(\xi-\frac{1}{2 \infty}\right)
$$

é uma outra função de Dirac infinitamente derivável distinta de $\delta_{0}$. Uma função de Dirac como $\delta_{+}$pode ser conveniente se pretendemos, por exemplo, aplicar a Transformada de Laplace, pois ela se anula para todo $\xi<0$, mas é normalizada no sentido:

$$
\int_{1 / \infty}^{3 / \infty} \delta_{+}(x) d x=1 .
$$

Não é difícil ver que:

Proposição: Se $\delta_{1}$ e $\delta_{2}$ são duas funções de Dirac então a função virtual $\delta_{3}: \overline{\mathbf{R}} \rightarrow \overline{\mathbf{R}}$ definida por:

$$
\delta_{3}(\xi)=\frac{1}{2}\left[\delta_{1}(\xi)+\delta_{2}(\xi)\right]
$$

também é uma função de Dirac. 


\subsection{Filtragem}

Usaremos daqui por diante a seguinte terminologia:

DefiniÇÃo: Diremos que uma função real é contínua em torno de $x \in \mathbf{R}$ quando existir um intervalo real aberto contendo $x$ no qual ela está definida e é contínua.

Temos então a propriedade de filtragem das funções de Dirac:

ProposiçÃo: Se $\delta: \overline{\mathbf{R}} \rightarrow \overline{\mathbf{R}}$ é uma função de Dirac, e $f: \mathbf{R} \rightarrow \mathbf{R}$ é uma função real contínua em torno da origem, então:

$$
\int_{-\infty}^{\infty} \delta(x) f(x) d x=f(0)
$$

Prova: Observamos inicialmente que existe um infinitésimo positivo $\varepsilon$ tal que $\delta(\xi)=0$, para todo $\xi$ tal que $|\xi| \geq \varepsilon$, pois $\delta$ é uma função de Dirac. Pela a aditividade com respeito ao intervalo de integração virtual, temos:

$$
\int_{-\infty}^{\infty} \delta(\xi) f(\xi) d \xi=\int_{-\infty}^{-\varepsilon} \delta(\xi) f(\xi) d \xi+\int_{-\varepsilon}^{\varepsilon} \delta(\xi) f(\xi) d \xi+\int_{\varepsilon}^{\infty} \delta(\xi) f(\xi) d \xi
$$

No membro direito da equação acima, os integrandos da primeira e da terceira integrais são identicamente nulos, qualquer que seja a função $f$, e portanto estas integrais existem e são iguais a zero. Na segunda integral (do membro direito) temos o produto de duas funções integráveis, logo a integral no membro esquerdo existe e:

$$
\int_{-\infty}^{\infty} \delta(\xi) f(\xi) d \xi=\int_{-\varepsilon}^{\varepsilon} \delta(\xi) f(\xi) d \xi
$$

É claro que vale, em $\mathbf{R}$, a seguinte afirmação: se $g$ e $h$ são duas funções reais definidas entre $-a$ e $a$, onde $a$ é um número real positivo, com $g$ integrável e nãonegativa e $h$ contínua, então existem dois números reais $c_{1}$ e $c_{2}$ entre $-a$ e $a$ tais que:

$$
h\left(c_{1}\right) \cdot \int_{-a}^{a} g(x) d x \leq \int_{-a}^{a} g(x) h(x) d x \leq h\left(c_{2}\right) \cdot \int_{-a}^{a} g(x) d x .
$$

Temos então, pelo TEV, que se $\varphi$ e $\psi$ são duas funções virtuais definidas entre $-\alpha$ e $\alpha$, onde $\alpha$ é um número virtual positivo, com $\varphi$ integrável e não-negativa e $\psi$ contínua, então existem dois números virtuais $\gamma_{1}$ e $\gamma_{2}$ entre $-\alpha$ e $\alpha$ tais que:

$$
\psi\left(\gamma_{1}\right) \cdot \int_{-\alpha}^{\alpha} \varphi(\xi) d \xi \leq \int_{-\alpha}^{\alpha} \varphi(\xi) \psi(\xi) d \xi \leq \psi\left(\gamma_{2}\right) \cdot \int_{-\alpha}^{\alpha} \varphi(\xi) d \xi
$$


Fazendo agora $\alpha=\varepsilon, \varphi=\delta$ e $\psi=f$, concluímos que existem dois números virtuais $\gamma_{1}$ e $\gamma_{2}$ entre $-\varepsilon$ e $\varepsilon$ tais que:

$$
f\left(\gamma_{1}\right) \cdot \int_{-\varepsilon}^{\varepsilon} \delta(\xi) d \xi \leq \int_{-\varepsilon}^{\varepsilon} \delta(\xi) f(\xi) d \xi \leq f\left(\gamma_{2}\right) . \int_{-\varepsilon}^{\varepsilon} \delta(\xi) d \xi
$$

Como $\gamma_{1}$ e $\gamma_{2}$ estão entre os infinitésimos $-\varepsilon$ e $\varepsilon$, eles próprios são infinitesimais. Segue então da continuidade de $f$ na origem que:

$$
f\left(\gamma_{1}\right) \approx f(0) \approx f\left(\gamma_{2}\right)
$$

Como:

$$
\int_{-\varepsilon}^{\varepsilon} \delta(\xi) d \xi \approx 1
$$

concluímos que:

$$
f\left(\gamma_{1}\right) \cdot \int_{-\varepsilon}^{\varepsilon} \delta(\xi) d \xi \approx f(0) \approx f\left(\gamma_{2}\right) \cdot \int_{-\varepsilon}^{\varepsilon} \delta(\xi) d \xi
$$

e portanto:

$$
\int_{-\infty}^{\infty} \delta(\xi) f(\xi) d \xi \approx f(0)
$$

Para demostrar a propriedade de filtragem na forma acima, fizemos uso essencial da condição de não-negatividade da função de Dirac. Esta condição evita as "unnecessarily wild variations" mencionadas por Dirac na sua caracterização da função delta (citada na introdução), que podem comprometer a filtragem de funções reais $f$ que se anulam na origem, como mostra o seguinte contra-exemplo trivial:

Seja $g_{a}: \mathbf{R} \rightarrow \mathbf{R}$ a família das funções reais dadas por:

$$
g_{a}(x)=\left\{\begin{array}{ll}
-a^{2} & , \text { se }-1 / a<x<0 \\
a^{2}+a & , \text { se } 0<x<1 / a \\
0 & , \text { caso contrário. }
\end{array} \quad(a>0)\right.
$$

Virtualizando o parâmetro $a$ obtemos, para cada $\alpha>\mathbf{R}$, uma função virtual $\delta_{\alpha}: \overline{\mathbf{R}} \rightarrow \overline{\mathbf{R}}$ que satisfaz as propriedades (i) e (ii) da definição de funções de Dirac, mas que assume valores negativos. Esta função virtual $\delta_{\alpha}$ não filtra corretamente a função identidade $f(x)=x$, pois:

$$
\begin{aligned}
\int_{-\infty}^{\infty} \delta_{\alpha}(\xi) f(\xi) d \xi & =\int_{-1 / \alpha}^{0}\left(-\alpha^{2}\right) \xi d \xi+\int_{0}^{1 / \alpha}\left(\alpha^{2}+\alpha\right) \xi d \xi \\
& =\left(-\alpha^{2}\right)\left[\frac{\xi^{2}}{2}\right]_{\xi=-1 / \alpha}^{\xi=0}+\left(\alpha^{2}+\alpha\right)\left[\frac{\xi^{2}}{2}\right]_{\xi=0}^{\xi=-1 / \alpha} \\
& =1+\frac{1}{2 \alpha} \\
& \approx 1
\end{aligned}
$$


isto é:

$$
\int_{-\infty}^{\infty} \delta_{\alpha}(x) f(x) d x=1 \neq f(0) .
$$

A propriedade de filtragem demonstrada acima vale para todas as funções de Dirac. Se estivermos trabalhando com uma função de Dirac particular, em um contexto específico, então podemos deduzir variantes desta propriedade por métodos análogos. Por exemplo, podemos usar a função $\delta_{+}$da seção anterior para filtrar unilateralmente uma função real $f$ definida apenas para valores positivos de $x$.

\subsection{Equivalência de Dirac}

Vejamos agora as regras operacionais em que se baseia o Cálculo Delta:

DefiniÇÃo: Diremos que duas funções virtuais integráveis $\varphi: \overline{\mathbf{R}} \rightarrow \overline{\mathbf{R}}$ e $\psi: \overline{\mathbf{R}} \rightarrow \overline{\mathbf{R}}$ são Dirac-equivalentes quando, para toda função real contínua $f: \mathbf{R} \rightarrow \mathbf{R}$, tivermos:

$$
\int_{-\infty}^{\infty} \varphi(x) f(x) d x=\int_{-\infty}^{\infty} \psi(x) f(x) d x .
$$

Nessa definição, ambas as integrais têm que ser redutíveis para que as duas funções sejam equivalentes. Ou seja, se $\varphi: \overline{\mathbf{R}} \rightarrow \overline{\mathbf{R}}$ é tal que existe uma função real contínua $f: \mathbf{R} \rightarrow \mathbf{R}$ que torna a integral virtual:

$$
\int_{-\infty}^{\infty} \varphi(\xi) f(\xi) d \xi
$$

irredutível, então $\varphi$ só equivale a ela mesma. Por exemplo, se $\delta$ é uma função de Dirac e $k \in \mathbf{R}$ uma constante real não nula, então a função soma $(\delta+k)$ só é equivalente a ela mesma, pois a integral:

$$
\int_{-\infty}^{\infty}[\delta(\xi)+k] d \xi=\int_{-\infty}^{\infty} \delta(\xi) d \xi+\int_{-\infty}^{\infty} k d \xi \approx 1+2 k \infty
$$

não é redutível.

No que segue, indicaremos que duas funções virtuais $\varphi$ e $\psi$ são Dirac-equivalentes escrevendo $\varphi(x) \equiv \psi(x)$, mas evitaremos a notação $\varphi(\xi) \equiv \psi(\xi)$. A variável real (letra latina, e não grega) enfatiza que as duas expressões são intercambiáveis apenas em integrais reduzidas. Por exemplo, escreveremos:

$$
\delta(2 x) \equiv \frac{1}{2} \delta(x)
$$

para toda função de Dirac $\delta$ (esta é uma consequência simples da regra de composição que será enunciada e provada na próxima seção). Este exemplo pode ser utilizado também 
para adquirirmos alguma intuição sobre a natureza dessa equivalência: o gráfico da função $\delta(2 x)$ tem "a mesma altura do gráfico da função $\delta$, mas metade da sua largura"; enquanto o da função $(1 / 2) \delta(x)$ tem "a mesma largura do gráfico da função $\delta$, mas metade da sua altura". Assim, apesar de bastante diferentes, estas funções equivalem como fatores em um integrando:

$$
\int_{-\infty}^{\infty} \delta(2 x) f(x) d x=\int_{-\infty}^{\infty} \frac{1}{2} \delta(x) f(x) d x=\frac{1}{2} f(0),
$$

pois ambas delimitam "a mesma área infinitamente concentrada sobre a origem", enquanto qualquer função real contínua $f$ "varia lentamente".

A definição seguinte será útil na discussão do papel da propriedade de filtragem na equivalência das funções de Dirac:

DefiniÇÃo: Diremos que uma função virtual integrável $\varphi: \overline{\mathbf{R}} \rightarrow \overline{\mathbf{R}}$ é filtrante quando, para toda função real contínua $f: \mathbf{R} \rightarrow \mathbf{R}$, tivermos:

$$
\int_{-\infty}^{\infty} \varphi(x) f(x) d x=f(0)
$$

Não é difícil ver que:

Proposição: O conjunto de todas as funções filtrantes é uma classe de equivalência segundo a relação de Dirac.

Toda função de Dirac é filtrante (como vimos na seção anterior), mas nem toda função virtual filtrante é uma função de Dirac. Para construir um contra-exemplo, podemos modificar em um único ponto a família parametrizada de funções $f_{a}$ utilizada na seç. 4.1 para definir a função de Dirac $\delta_{0}$ :

$$
g_{a}(x)= \begin{cases}f_{a}(x) & , \text { se } x \neq 7 \\ 3 & , \text { se } x=7\end{cases}
$$

Virtualizando o parâmetro de $g_{a}$ para $\infty$ obtemos uma função virtual $\varphi: \overline{\mathbf{R}} \rightarrow \overline{\mathbf{R}}$ que é claramente filtrante, mas que não é uma função de Dirac, pois $\varphi(7)=3$.

Vemos então que:

Proposição: O conjunto de todas as funções de Dirac está contido propriamente na classe de equivalência (segundo a relação de Dirac) das funções filtrantes.

Como corolário:

ProposiçÃo: Duas funções de Dirac quaisquer são sempre equivalentes entre si. 
Esse fato compatibiliza duas convenções que fazem parte da linguagem tradicional do Cálculo Delta:

(i) Todas as funções de Dirac são representadas por um mesmo símbolo: " $\delta$ ".

(ii) A equivalência de Dirac é indicada simplesmente pelo símbolo de igualdade: " =" . Estas convenções simplificam drasticamente a notação, e é claro que não comprometem o rigor de um trabalho científico se forem usadas criteriosamente. Entretanto, como elas podem gerar alguma confusão, continuaremos a distinguir claramente igualdade $(=)$ de equivalência ( $\equiv$ ), e a representar funções de Dirac distintas por símbolos distintos.

A linguagem tradicional do Cálculo Delta também não estabelece uma distinção clara entre funções filtrantes e funções de Dirac, uma vez que estas são geralmente manipuladas através da propriedade de filtragem. Isto também pode causar mal-entendidos, principalmente quando as convenções acima estão sendo utilizadas.

\subsection{A Regra de Composição}

As funções de Dirac, como definidas na seç. 4.1, são apenas funções virtuais $\delta: \overline{\mathbf{R}} \rightarrow$ $\overline{\mathbf{R}}$ com certas características específicas. Está claro, portanto, que elas podem ser compostas com qualquer outra função virtual $\varphi$, sendo que a função virtual composta $(\delta \circ \varphi)$ tem o mesmo domínio de $\varphi$.

Muitas das regras operacionais do Cálculo Delta envolvem a composição de uma função de Dirac $\delta$ com uma função real $g$. Para discutir estas equivalências de Dirac, vamos supor que a função real $g$ está definida em toda a reta real, de modo que a função virtual composta $(\delta \circ g)$ está definida em todo $\xi \in \overline{\mathbf{R}}$.

As funções de Dirac só não se anulam nas proximidades da origem. Assim, se $g(x)$ não se aproxima da origem então $\delta[g(x)]=0$. Em outras palavras:

Proposição: Seja $\delta: \overline{\mathbf{R}} \rightarrow \overline{\mathbf{R}}$ uma função de Dirac e $g: \mathbf{R} \rightarrow \mathbf{R}$ uma função real com a seguinte propriedade: existe um número real $r>0$ tal que $|g(x)|>r$, para todo $x \in \mathbf{R}$. Então a função composta $(\delta \circ g): \overline{\mathbf{R}} \rightarrow \overline{\mathbf{R}}$ é identicamente nula:

$$
\delta[g(\xi)]=0, \quad \text { para todo } \xi \in \overline{\mathbf{R}} .
$$

Nesse caso, é claro que $\delta[g(x)] \equiv 0$. Por exemplo:

$$
\delta\left(x^{2}+1\right) \equiv 0 \quad \text { e } \quad \delta(\operatorname{sen} x+2) \equiv 0 .
$$

Consideremos agora uma função real cuja imagem se aproxima da origem: $g(x)=x^{2}$, por exemplo. Se tomarmos a função de Dirac $\delta_{0}$ definida na seç. 4.1, e definirmos:

$$
\delta_{-}(\xi)=\delta_{0}(\xi+2 / \infty)
$$


então teremos $\delta_{-}\left(\xi^{2}\right)=0$, para todo $\xi \in \overline{\mathbf{R}}$, pois $\delta_{-}(\xi)=0$, para todo $\xi \geq 0$. É claro então que $\delta_{-}\left(x^{2}\right) \equiv 0$. Por outro lado, compondo a função de Dirac "quadrada" $\delta_{\sqcap}$ (definida na seç. 4.1) com esta mesma função real $g(x)=x^{2}$, teremos uma composta não-nula:

$$
|\xi|<\frac{1}{\sqrt{\infty}} \quad \text { implica } \quad \delta_{\sqcap}\left(\xi^{2}\right)=\frac{\infty}{2} .
$$

Além disto, é fácil ver que $\delta_{\sqcap}\left(x^{2}\right) \not \equiv 0$, pois:

$$
\int_{-\infty}^{\infty} \delta_{\sqcap}\left(\xi^{2}\right) d \xi=\int_{-1 / \sqrt{\infty}}^{1 / \sqrt{\infty}} \frac{\infty}{2} d \xi=\sqrt{\infty} .
$$

Temos então que $\delta_{-}\left(x^{2}\right) \not \equiv \delta_{\sqcap}\left(x^{2}\right)$, o que nos mostra que a classe de equivalência da composta $\delta\left(x^{2}\right)$ depende da função de Dirac particular escolhida. Ou seja, não existe uma função virtual $\varphi$ tal que $\delta\left(x^{2}\right) \equiv \varphi(x)$, para toda função de Dirac $\delta$. Portanto, não há uma regra operacional para $\delta\left(x^{2}\right)$ na linguagem tradicional do Cálculo Delta, que só trabalha com equivalências genéricas, válidas para todas as funções de Dirac.

Essa dependência da classe da composta $\delta[g(x)]$ com relação à escolha da função de Dirac $\delta$ também pode ocorrer se o gráfico da função real $g: \mathbf{R} \rightarrow \mathbf{R}$ assintotar o eixo $x$, como, por exemplo, para $g(x)=e^{x}$. Entretanto, a classe da função composta será independente da função de Dirac escolhida se o gráfico da função real $g: \mathbf{R} \rightarrow \mathbf{R}$ apenas "cruzar" o eixo $x$ em um número finito de raízes, sem se aproximar dele em qualquer outra região:

DefiniçÃo: Diremos que uma função real $g: \mathbf{R} \rightarrow \mathbf{R}$ é simples quando ela possuir apenas um número finito de raízes $a_{1}, a_{2}, \ldots, a_{n}$ e existir um número real $r>0$ com as seguintes propriedades:

(i) os $n$ intervalos $\left[a_{i}-r, a_{i}+r\right]$ são disjuntos dois-a-dois e $|g(x)|>r$, para todo $x$ fora destes intervalos;

(ii) dentro dos $n$ intervalos $\left[a_{i}-r, a_{i}+r\right]$ a função $g$ é derivável, sua derivada é contínua e não se anula.

Lema: Se $\delta: \overline{\mathbf{R}} \rightarrow \overline{\mathbf{R}}$ é uma função de Dirac qualquer e $g: \mathbf{R} \rightarrow \mathbf{R}$ uma função real simples, então, para toda função real $f: \mathbf{R} \rightarrow \mathbf{R}$ contínua em torno das raízes de $g$, temos:

$$
\int_{-\infty}^{\infty} \delta[g(x)] f(x) d x=\sum_{i=1}^{n} \frac{f\left(a_{i}\right)}{\left|g^{\prime}\left(a_{i}\right)\right|} .
$$

Prova: Como a função $g$ é simples, ela possui apenas um número finito de raízes $a_{1}, \ldots, a_{n}$ e existe um número real $r>0$ tal que a composta $\delta[g(\xi)]$ se anula identicamente fora dos $n$ intervalos $\left[a_{i}-r, a_{i}+r\right]$. Portanto:

$$
\int_{-\infty}^{\infty} \delta[g(\xi)] f(\xi) d \xi=\sum_{i=1}^{n} \int_{a_{i}-r}^{a_{i}+r} \delta[g(\xi)] f(\xi) d \xi
$$


Vamos calcular separadamente as integrais do somatório acima, observando inicialmente que, para cada $i=1,2, \ldots, n$, a restrição da função $g$ ao intervalo $\left[a_{i}-r, a_{i}+r\right]$ admite uma inversa $h_{i}$ :

$$
h_{i}[g(x)]=x, \quad \text { para todo } x \in\left[a_{i}-r, a_{i}+r\right]
$$

e que esta inversa $h_{i}$ é derivável e sua derivada não se anula entre $g\left(a_{i}+r\right)$ e $g\left(a_{i}-r\right)$. Fazendo então (para cada $i=1,2, \ldots, n$ ) a mudança de variáveis:

$$
\mu=g(\xi), \quad \xi=h_{i}(\mu) \quad \text { e } \quad d \mu=g^{\prime}(\xi) d \xi
$$

obtemos:

$$
\int_{a_{i}-r}^{a_{i}+r} \delta[g(\xi)] f(\xi) d \xi=\int_{a_{i}-r}^{a_{i}+r} \delta[g(\xi)] \frac{f(\xi)}{g^{\prime}(\xi)} g^{\prime}(\xi) d \xi=\int_{g\left(a_{i}-r\right)}^{g\left(a_{i}+r\right)} \delta(\mu) \frac{f\left[h_{i}(\mu)\right]}{g^{\prime}\left[h_{i}(\mu)\right]} d \mu .
$$

Se $g^{\prime}\left(a_{i}\right)>0$ então $g\left(a_{i}-r\right)<g\left(a_{i}+r\right)$, e a propriedade de filtragem nos garante que:

$$
\int_{g\left(a_{i}-r\right)}^{g\left(a_{i}+r\right)} \delta(\mu) \frac{f\left[h_{i}(\mu)\right]}{g^{\prime}\left[h_{i}(\mu)\right]} d \mu \approx \frac{f\left[h_{i}(0)\right]}{g^{\prime}\left[h_{i}(0)\right]}=\frac{f\left(a_{i}\right)}{g^{\prime}\left(a_{i}\right)}
$$

Por outro lado, se $g^{\prime}\left(a_{i}\right)<0$ então $g\left(a_{i}-r\right)>g\left(a_{i}+r\right)$, e a propriedade de filtragem nos fornece:

$$
\int_{g\left(a_{i}-r\right)}^{g\left(a_{i}+r\right)} \delta(\mu) \frac{f\left[h_{i}(\mu)\right]}{g^{\prime}\left[h_{i}(\mu)\right]} d \mu \approx-\frac{f\left[h_{i}(0)\right]}{g^{\prime}\left[h_{i}(0)\right]}=-\frac{f\left(a_{i}\right)}{g^{\prime}\left(a_{i}\right)} .
$$

Assim, em qualquer caso temos:

$$
\int_{a_{i}-r}^{a_{i}+r} \delta[g(\xi)] f(\xi) d \xi \approx \frac{f\left(a_{i}\right)}{\left|g^{\prime}\left(a_{i}\right)\right|}
$$

Portanto:

$$
\int_{-\infty}^{\infty} \delta[g(x)] f(x) d x=\sum_{i=1}^{n} \frac{f\left(a_{i}\right)}{\left|g^{\prime}\left(a_{i}\right)\right|}
$$

Para qualquer número $a \in \mathbf{R}$, a função real dada por $g(x)=x-a$ é simples. O lema anterior nos fornece então a propriedade de filtragem das funções de Dirac transladadas:

Proposição: Se $\delta: \overline{\mathbf{R}} \rightarrow \overline{\mathbf{R}}$ é uma função de Dirac qualquer e $f: \mathbf{R} \rightarrow \mathbf{R}$ uma função contínua em torno de $a \in \mathbf{R}$, então:

$$
\int_{-\infty}^{\infty} \delta(x-a) f(x) d x=f(a) .
$$

Chegamos assim à regra operacional mais importante do Cálculo Delta: 
Regra de Composição: Se $\delta: \overline{\mathbf{R}} \rightarrow \overline{\mathbf{R}}$ é uma função de Dirac qualquer e $g: \mathbf{R} \rightarrow \mathbf{R}$ uma função real simples de raízes $a_{1}, a_{2}, \ldots, a_{n}$, então:

$$
\delta[g(x)] \equiv \sum_{i=1}^{n} \frac{1}{\left|g^{\prime}\left(a_{i}\right)\right|} \delta\left(x-a_{i}\right) .
$$

Prova: Seja $f: \mathbf{R} \rightarrow \mathbf{R}$ uma função real contínua. Aplicando a proposição acima a cada $f\left(a_{i}\right)$ do somatório presente no enunciado do lema anterior, obtemos:

$$
\begin{aligned}
\int_{-\infty}^{\infty} \delta[g(x)] f(x) d x & =\sum_{i=1}^{n} \frac{1}{\left|g^{\prime}\left(a_{i}\right)\right|} \int_{-\infty}^{\infty} \delta\left(x-a_{i}\right) f(x) d x \\
& =\int_{-\infty}^{\infty}\left[\sum_{i=1}^{n} \frac{1}{\left|g^{\prime}\left(a_{i}\right)\right|} \delta\left(x-a_{i}\right)\right] f(x) d x
\end{aligned}
$$

Como casos particulares da regra de composição, temos:

$$
\delta(a x) \equiv \frac{1}{|a|} \delta(x) \quad(a \neq 0),
$$

e

$$
\delta\left(x^{2}-a^{2}\right) \equiv \frac{1}{2 a}[\delta(x-a)+\delta(x+a)] \quad(a>0),
$$

para toda função de Dirac $\delta$.

Usando a propriedade de filtragem das funções de Dirac transladadas é fácil ver também que:

Proposição: Se $\delta: \overline{\mathbf{R}} \rightarrow \overline{\mathbf{R}}$ é uma função de Dirac qualquer e $f: \mathbf{R} \rightarrow \mathbf{R}$ uma função real contínua em torno de $a \in \mathbf{R}$, então:

$$
f(x) \delta(x-a) \equiv f(a) \delta(x-a)
$$

\subsection{Contração de Funções de Dirac}

Quando se faz uma analogia formal entre as somas finitas da álgebra linear (com somandos indexados por um índice discreto) e somas infinitas representadas por integrais (com somandos indexados por um variável contínua), a função delta corresponde ao símbolo de Kronecker $\delta_{\alpha}^{\beta}$ da notação tensorial clássica. Esta correspondência formal, que ocorre claramente no contexto da Mecânica Quântica, não apenas motivou Dirac a escolher a letra grega $\delta$ para representar suas funções como também serviu de guia no estabelecimento 
de muitas de suas propriedades. Por exemplo, o delta de Kronecker é invariante por contrações:

$$
\sum_{\beta} \delta_{\alpha}^{\beta} \delta_{\beta}^{\gamma}=\delta_{\alpha}^{\gamma}
$$

As funções de Dirac contínuas têm uma propriedade correspondente:

$$
\int_{-\infty}^{\infty} \delta_{1}(\xi-\beta) \delta_{2}(\beta-\alpha) d \beta=\delta_{3}(\xi-\alpha),
$$

isto é, a contração de duas funções de Dirac contínuas também é uma função de Dirac. O lema seguinte será utilizado na demonstração desse resultado:

Lema: Se $\delta: \overline{\mathbf{R}} \rightarrow \overline{\mathbf{R}}$ é uma função de Dirac, e $\beta \in \overline{\mathbf{R}}$ um número virtual finito, então:

$$
\int_{-\infty}^{\infty} \delta(\xi-\beta) d \xi=\int_{-\infty}^{\infty} \delta(\xi) d \xi
$$

Prova: Fazendo a mudança de variáveis $\mu=\xi-\beta$ obtemos:

$$
\begin{aligned}
\int_{-\infty}^{\infty} \delta(\xi-\beta) d \xi & =\int_{-\infty-\beta}^{\infty-\beta} \delta(\mu) d \mu \\
& =\int_{-\infty-\beta}^{-\infty} \delta(\mu) d \mu+\int_{-\infty}^{\infty} \delta(\mu) d \mu+\int_{\infty}^{\infty-\beta} \delta(\mu) d \mu
\end{aligned}
$$

mas os integrandos da primeira e da terceira integrais acima são identicamente nulos, pois $\beta$ é finito e $\delta$ é uma função de Dirac.

Proposição: Se $\delta_{1}$ e $\delta_{2}$ são duas funções de Dirac contínuas então a função virtual $\delta_{3}: \overline{\mathbf{R}} \rightarrow \overline{\mathbf{R}}$ definida por:

$$
\delta_{3}(\xi)=\int_{-\infty}^{\infty} \delta_{1}(\xi-\beta) \delta_{2}(\beta) d \beta
$$

também é uma função de Dirac. Além disto, temos que:

$$
\int_{-\infty}^{\infty} \delta_{1}(\xi-\beta) \delta_{2}(\beta-\alpha) d \beta=\delta_{3}(\xi-\alpha),
$$

para todo $\alpha \in \overline{\mathbf{R}}$ finito.

Prova: É fácil ver que $\delta_{3}(\xi) \geq 0$, para todo $\xi \in \overline{\mathbf{R}}$, pois o integrando acima é nãonegativo. Para mostrar que $\delta_{3}(\xi)$ se anula longe da origem, observamos inicialmente que existem infinitésimos positivos $\varepsilon_{1}$ e $\varepsilon_{2}$ tais que:

$$
|\xi| \geq \varepsilon_{1} \text { implica } \delta_{1}(\xi)=0 \quad \text { e } \quad|\xi| \geq \varepsilon_{2} \text { implica } \delta_{2}(\xi)=0 \text {. }
$$


Fazendo $\varepsilon_{3}=\varepsilon_{1}+\varepsilon_{2}$ obtemos:

$$
|\xi| \geq \varepsilon_{3} \text { implica } \delta_{3}(\xi)=0
$$

Para demonstrar que $\delta_{3}$ é integrável e está normalizada:

$$
\int_{-\infty}^{\infty} \delta_{3}(\xi)=\int_{-\infty}^{\infty}\left[\int_{-\infty}^{\infty} \delta_{1}(\xi-\beta) \delta_{2}(\beta) d \beta\right] d \xi=1
$$

consideremos a seguinte afirmação em $\mathbf{R}$ : se $a_{1}$ e $a_{2}$ são dois números reais positivos; $g_{1}$ é uma função real definida e contínua entre $-a_{1}$ e $a_{1}$, e tal que, para todo $b$ entre $-a_{2}$ e $a_{2}$ :

$$
\int_{-a_{1}}^{a_{1}} g_{1}(x-b) d x=\int_{-a_{1}}^{a_{1}} g_{1}(x) d x
$$

e $g_{2}$ é uma função real definida e contínua entre $-a_{2}$ e $a_{2}$; então:

$$
\int_{-a_{1}}^{a_{1}}\left[\int_{-a_{2}}^{a_{2}} g_{1}(x-b) g_{2}(b) d b\right] d x=\left[\int_{-a_{1}}^{a_{1}} g_{1}(x) d x\right]\left[\int_{-a_{2}}^{a_{2}} g_{2}(b) d b\right] .
$$

Esta afirmação pode ser facilmente demonstrada em $\mathbf{R}$ pelo Teorema de Fubini, e então estendida para $\overline{\mathbf{R}}$ pelo TEV: e $\alpha_{1}$ e $\alpha_{2}$ são dois números virtuais positivos; $\varphi_{1}$ é uma função virtual definida e contínua entre $-\alpha_{1}$ e $\alpha_{1}$, e tal que, para todo $\beta$ entre $-\alpha_{2}$ e $\alpha_{2}$ :

$$
\int_{-\alpha_{1}}^{\alpha_{1}} \varphi_{1}(\xi-\beta) d \xi=\int_{-\alpha_{1}}^{\alpha_{1}} \varphi_{1}(\xi) d \xi
$$

e $\varphi_{2}$ é uma função virtual definida e contínua entre $-\alpha_{2}$ e $\alpha_{2}$; então:

$$
\int_{-\alpha_{1}}^{\alpha_{1}}\left[\int_{-\alpha_{2}}^{\alpha_{2}} \varphi_{1}(\xi-\beta) \varphi_{2}(\beta) d \beta\right] d \xi=\left[\int_{-\alpha_{1}}^{\alpha_{1}} \varphi_{1}(\xi) d \xi\right]\left[\int_{-\alpha_{2}}^{\alpha_{2}} \varphi_{2}(\beta) d \beta\right]
$$

Fazendo agora

$$
\int_{-\infty}^{\infty} \delta_{3}(\xi)=\int_{-\infty}^{\infty}\left[\int_{-\varepsilon_{2}}^{\varepsilon_{2}} \delta_{1}(\xi-\beta) \delta_{2}(\beta) d \beta\right] d \xi
$$

o lema acima nos garante que tomando $\alpha_{1}=\infty, \alpha_{2}=\varepsilon_{2}, \varphi_{1}=\delta_{1}$ e $\varphi_{2}=\delta_{2}$ obtemos:

$$
\int_{-\infty}^{\infty} \delta_{3}(\xi) d \xi=\left[\int_{-\infty}^{\infty} \delta_{1}(\xi) d \xi\right]\left[\int_{-\varepsilon_{2}}^{\varepsilon_{2}} \delta_{2}(\beta) d \beta\right] \approx 1 .
$$

Está demonstrado então que $\delta_{3}$ é, de fato, uma função de Dirac. Para calcular a integral:

$$
\int_{-\infty}^{\infty} \delta_{1}(\xi-\beta) \delta_{2}(\beta-\alpha) d \beta
$$


fazemos a mudança de variáveis $\mu=\beta-\alpha$ :

$$
\begin{aligned}
\int_{-\infty}^{\infty} \delta_{1}(\xi-\beta) \delta_{2}(\beta-\alpha) d \beta & =\int_{-\infty-\alpha}^{\infty-\alpha} \delta_{1}(\xi-\alpha-\mu) \delta_{2}(\mu) d \mu \\
& =\int_{-\infty}^{\infty} \delta_{1}(\xi-\alpha-\beta) \delta_{2}(\mu) d \mu \\
& =\delta_{3}(\xi-\alpha)
\end{aligned}
$$

Esse resultado nos mostra que:

$$
\int_{-\infty}^{\infty} \delta(x-\beta) \delta(\beta-a) d \beta \equiv \delta(x-a)
$$

\subsection{Funções de Dirac Diferenciáveis}

Como vimos na seç. 4.1, algumas funções de Dirac são deriváveis em todo $\xi \in \overline{\mathbf{R}}$, outras não. Vamos agora discutir a propriedade de filtragem associada às derivadas de funções de Dirac.

Definição: Diremos que uma função real é diferenciável em torno de $x \in \mathbf{R}$ quando existir um intervalo real aberto contendo $x$ no qual ela está definida, é derivável, e sua derivada é uma função contínua. Além disso, diremos simplesmente que uma função é diferenciável quando ela for diferenciável em todos os pontos do seu domínio. A extensão virtual deste atributo de funções reais define a diferenciabilidade de funções virtuais.

Segundo essa definição, uma função virtual $\varphi=\left\langle f_{1}, f_{2}, f_{3}, \ldots\right\rangle$ é diferenciável quando os objetos $f_{i} \in \mathcal{U}$ forem funções diferenciáveis a partir de um certo valor do índice $i$.

Proposição: Se $\delta: \overline{\mathbf{R}} \rightarrow \overline{\mathbf{R}}$ é uma função de Dirac diferenciável, e $f: \mathbf{R} \rightarrow \mathbf{R}$ é uma função real diferenciável em torno de $a \in \mathbf{R}$, então:

$$
\int_{-\infty}^{\infty} \delta^{\prime}(x-a) f(x) d x=-f^{\prime}(a)
$$

Prova: Como $\delta$ é uma função de Dirac, existe um infinitésimo positivo $\varepsilon$ tal que $|\xi| \geq \varepsilon$ implica $d t(\xi)=0$. É claro que $\delta^{\prime}(\xi)=0$, para todo $|\xi| \geq \varepsilon$. Pela aditividade com respeito ao intervalo de integração virtual, temos:

$\int_{-\infty}^{\infty} \delta^{\prime}(\xi-a) f(\xi) d \xi=\int_{-\infty}^{a-\varepsilon} \delta^{\prime}(\xi-a) f(\xi) d \xi+\int_{a-\varepsilon}^{a+\varepsilon} \delta^{\prime}(\xi-a) f(\xi) d \xi+\int_{a+\varepsilon}^{\infty} \delta^{\prime}(\xi-a) f(\xi) d \xi$.

No membro direito desta equação, os integrandos da primeira e da terceira integrais são identicamente nulos, qualquer que seja a função $f$, e portanto estas integrais existem e são 
iguais a zero. Na segunda integral (do membro direito) temos o produto de duas funções contínuas, logo a integral no membro esquerdo existe e:

$$
\int_{-\infty}^{\infty} \delta^{\prime}(\xi-a) f(\xi) d \xi=\int_{a-\varepsilon}^{a+\varepsilon} \delta^{\prime}(\xi-a) f(\xi) d \xi .
$$

O TEV nos mostra que a fórmula de integração por partes vale para integrais virtuais. Como a função $f: \mathbf{R} \rightarrow \mathbf{R}$ é diferenciável entre $a-\varepsilon$ e $a+\varepsilon$, temos:

$$
\begin{aligned}
\int_{a-\varepsilon}^{a+\varepsilon} \delta^{\prime}(\xi-a) f(\xi) d \xi & =[\delta(\xi-a) f(\xi)]_{a-\varepsilon}^{a+\varepsilon}-\int_{a-\varepsilon}^{a+\varepsilon} \delta(\xi-a) f^{\prime}(\xi) d \xi \\
& =\int_{a-\varepsilon}^{a+\varepsilon} \delta(\xi-a)\left[-f^{\prime}(\xi)\right] d \xi \\
& =\int_{-\infty}^{\infty} \delta(\xi-a)\left[-f^{\prime}(\xi)\right] d \xi \\
& \approx-f^{\prime}(a) .
\end{aligned}
$$

Como corolário desse resultado:

Proposição: Se $\delta: \overline{\mathbf{R}} \rightarrow \overline{\mathbf{R}}$ é uma função de Dirac diferenciável então sua derivada $\delta^{\prime}: \overline{\mathbf{R}} \rightarrow \overline{\mathbf{R}}$ não pode ser uma função de Dirac.

Prova: Tomando na proposição anterior $a=0$ e $f(x)$ constantemente igual a 1 , obtemos:

$$
\int_{-\infty}^{\infty} \delta^{\prime}(x) d x=0
$$

Portanto $\delta^{\prime}$ não pode ser uma função de Dirac.

Seguindo a mesma linha de argumentação acima, não é difícil chegar à propriedade de filtragem associada às derivadas de ordem superior de uma função de Dirac suficientemente diferenciável:

Proposição: Se $\delta: \overline{\mathbf{R}} \rightarrow \overline{\mathbf{R}}$ é uma função de Dirac $n$ vezes diferenciável, e $f: \mathbf{R} \rightarrow \mathbf{R}$ é uma função real $n$ vezes diferenciável em torno de $a \in \mathbf{R}$, então:

$$
\int_{-\infty}^{\infty} \delta^{(n)}(x-a) f(x) d x=(-1)^{n} f^{(n)}(a) .
$$

Essa propriedade de filtragem das derivadas de ordem superior de uma função de Dirac só valem para funções reais suficientemente diferenciáveis, enquanto a equivalência de Dirac, como definida na seç. 4.3, exige que:

$$
\int_{-\infty}^{\infty} \varphi(x) f(x) d x=\int_{-\infty}^{\infty} \psi(x) f(x) d x
$$


para toda função real contínua $f: \mathbf{R} \rightarrow \mathbf{R}$. Como há funções reais contínuas não deriváveis, não podemos deduzir fórmulas de equivalência neste sentido "forte" usando as propriedades acima, mesmo para uma função de Dirac suficientemente diferenciável. Entretanto, se estivermos trabalhando apenas com funções reais diferenciáveis, podemos considerar equivalências de Dirac "fracas", que exigem que as integrais sejam iguais apenas para funções reais deste tipo:

DefiniçÃo: Diremos que duas funções virtuais integráveis $\varphi: \overline{\mathbf{R}} \rightarrow \overline{\mathbf{R}}$ e $\psi: \overline{\mathbf{R}} \rightarrow \overline{\mathbf{R}}$ são Dirac-equivalentes em ordem $n$ quando, para toda função real $n$ vezes diferenciável $f: \mathbf{R} \rightarrow \mathbf{R}$, tivermos:

$$
\int_{-\infty}^{\infty} \varphi(x) f(x) d x=\int_{-\infty}^{\infty} \psi(x) f(x) d x .
$$

Indicaremos que duas funções virtuais são Dirac-equivalentes em ordem $n$ escrevendo:

$$
\varphi(x) \equiv \psi(x) \quad(\text { ordem } n)
$$

Com essa definição, não é difícil deduzir da proposição anterior que:

Proposição: Se $\delta: \overline{\mathbf{R}} \rightarrow \overline{\mathbf{R}}$ é uma função de Dirac $n$ vezes diferenciável e $g: \mathbf{R} \rightarrow \mathbf{R}$ uma função real $n$ vezes diferenciável em torno de $a \in \mathbf{R}$, então:

$$
\left.g(x) \delta^{(n)}(x-a) \equiv(-1)^{n} \sum_{i=0}^{n}(-1)^{i}\left(\begin{array}{c}
n \\
i
\end{array}\right) g^{(n-i)}(a) \delta^{(i)}(x-a) \quad \quad \text { (ordem } n\right) .
$$




\section{Epílogo}

Esperamos ter demonstrado que é perfeitamente possível trabalhar com o conceito de processo matemático sem utilizar uma noção subjetiva de tempo, que poderia acarretar mal-entendidos e contradições. Vimos que, para tanto, não precisamos de nada além dos métodos elementares da Teoria dos Conjuntos.

Esperamos também ter deixado claro que este caminho nos permite superar as limitações do paradigma weierstrasiano, sem abrir mão do rigor formal que ele introduziu na Análise Matemática. Até onde sabemos, este trabalho apresenta (seç. 4.4) a primeira demonstração contextualizada e rigorosa (segundo os padrões da matemática atual) da regra operacional básica associada à função delta de Dirac, a regra de composição:

$$
\delta[g(x)]=\sum_{i=1}^{n} \frac{1}{\left|g^{\prime}\left(a_{i}\right)\right|} \delta\left(x-a_{i}\right) .
$$

Neste caso, teríamos aqui a primeira formalização matemática das idéias essenciais do Cálculo Delta.

O processo de extensão virtual pode ser usado como base de futuras investigações puramente matemáticas. Por exemplo: o conceito tradicional de espaço topológico decorreu, historicamente, da definição de Weierstrass para a noção de continuidade de funções reais - os conjuntos abertos de uma topologia nada mais são do que épsilons e deltas generalizados. Analogamente, a organização do Cálculo apresentada no cap. 2 deste trabalho sugere a seguinte generalização:

DEFINIÇÃO: Uma topologia virtual sobre um conjunto A é uma relação de equivalência $\approx$ sobre sua extensão virtual $\overline{\mathrm{A}}$. Um espaço topológico virtual é um conjunto munido de uma topologia virtual. 
Essa definição é mais simples, mais direta e mais intuitiva que a definição tradicional de topologia. Ela permite, por exemplo, a seguinte caracterização da continuidade de funções entre dois espaços topológicos virtuais A e B:

DeFiniçÃo: Uma função $f: \mathrm{A} \rightarrow \mathrm{B}$ é contínua $\mathrm{em} \quad x \in \mathrm{A}$ quando $\alpha \approx \bar{x}$ implica $\bar{f}(\alpha) \approx \bar{f}(\bar{x})$.

Além disso, é trivial definir continuidade uniforme neste contexto:

DEFINIÇÃO: Uma função $f: \mathrm{A} \rightarrow \mathrm{B}$ é uniformemente contínua quando $\alpha \approx \beta$ implica $\bar{f}(\alpha) \approx \bar{f}(\beta)$.

Sabemos que não é possível definir continuidade uniforme utilizando-se apenas a estrutura topológica tradicional. Do nosso ponto de vista, a desajeitada definição de uniformidade nos parece uma evidência interna das limitações do paradigma weierstrasiano.

No entanto, acreditamos que seria prematuro lançarmo-nos na investigação das propriedades puramente matemáticas dos espaços topológicos virtuais, uma vez que o processo de extensão virtual só foi utilizado concretamente, até o presente momento, na formalização do Cálculo Delta de Dirac, que não levantou questões matemáticas suficientes para justificar tal investigação.

Assim, pretendemos agora usar o formalismo geral das extensões virtuais, que foi apresentado neste trabalho, como ferramenta matemática no estudo de idéias que sejam relevantes para o Conhecimento Científico como um todo. Parece-nos que o conceito de integral de trajetória de Feynman se destaca entre tais idéias, tendo em vista tanto as dificuldades matemáticas encontradas no seu estudo quanto a posição central que a Mecânica Quântica ocupa atualmente nos fundamentos da Física. 


\section{Apêndice}

Apresentamos aqui uma tradução para o Português dos fragmentos de texto citados neste trabalho:

CitAÇÃO DA PÁG. 2:

O afastamento em relação à ciência se acelerou durante este século. Hoje em dia é comum se ouvir e se ler matemáticos declarando independência em relação à ciência. Matemáticos não hesitam mais em falar abertamente sobre seu interesse exclusivo por matemática propriamente dita, e sobre sua indiferença à ciência. Apesar de não haver estatísticas precisas disponíveis, aproximadamente noventa por cento dos matemáticos atualmente ativos são ignorantes em ciência e estão contentes em permanecer neste estado de graça. A despeito da história e de alguma oposição, continuou a tendência à abstração, à generalização pela generalização, e à investigação de problemas escolhidos arbitrariamente. A necessidade, perfeitamente razoável, de se estudar toda uma classe de problemas a fim de captar a essência de um problema particular tornou-se uma desculpa para a investigação de generalidades e abstrações sem maiores justificativas.

Ao longo dos séculos, o homem criou obras grandiosas como a geometria euclideana, a teoria ptolomaica, a teoria heliocêntrica, a mecânica newtoniana, a teoria eletromagnética, e, mais recentemente, as teorias quântica e da relatividade. Sabemos que a matemática é o método de construção, a estrutura, e mesmo a própria essência destas e de outras obras científicas significativas e poderosas. As teorias matemáticas nos permitiram conhecer algo da natureza, englobar em descrições abrangentes e inteligíveis uma grande variedade de fenmenos aparentemente diversos. As teorias matemáticas revelaram a ordem e o plano encontrados na natureza, quaisquer que eles fossem, e nos deram controle, total ou parcial, 
sobre vastos domínios.

Mas a maioria dos matemáticos abandonou suas tradições e sua herança. As mensagens grávidas que a natureza envia aos sentidos agora encontram olhos fechados e ouvidos desatentos. Os matemáticos estão vivendo da reputação adquirida pelos seus predecessores, e ainda esperam a aclamação e o apoio que os trabalhos anteriores asseguraram. Os matemáticos puros foram além. Eles expulsaram os matemáticos aplicados da sua fraternidade, na esperança de, açambarcando o honorável título de matemático, receberem sozinhos a glória devida a seus predecessores. Eles jogaram fora sua rica fonte de idéias e agora estão gastando sua riqueza previamente acumulada. Eles seguiram um brilho que os levou para fora deste mundo. É verdade que alguns, atentos à nobre tradição que motivou a pesquisa matemática no passado e garantiu a honra atribuída aos Newtons e Gausses, ainda alegam um valor potencial de seus trabalhos matemáticos para a ciência. Eles falam em criar modelos para a ciência. Mas na verdade não estão preocupados com esta meta. De fato, como a maioria do matemáticos modernos não conhece ciência nenhuma, eles não podem estar criando modelos. Eles preferem permanecer virgens a ir para a cama com a ciência. Como um todo, a matemática está agora voltada para dentro de si mesma; alimentando-se de si mesma; e é extremamente improvável, a julgar pelo que aconteceu no passado, que a maior parte da pesquisa matemática moderna irá algum dia contribuir para o avanço da ciência; a matemática pode estar fadada a tatear na escuridão. A matemática é hoje um empreendimento quase inteiramente auto-suficiente. Movendo-se em direções determinadas pelos seus próprios critérios de relevância e excelência, está até mesmo orgulhosa de sua independência em relação a problemas, motivações e inspirações externas. Ela não tem mais unidade e propósito.

CitAÇÃO DA PÁG. 6:

Introduzir funções ideais pode parecer uma ampla extensão do Cálculo ordinário. Entretanto, no âmbito das funções ideais, nem toda operação do Cálculo clássico pode ser realizada. Assim, a vantagem de se assegurar diferenciabilidade irrestrita é parcialmente contrabalançada pela perda da liberdade de multiplicar funções e de formar funções compostas. Não é nem mesmo completamente verdade que uma função ideal de várias variáveis se torna uma função ideal de um número menor variáveis se algumas delas são mantidas constantes em um domínio de definição.

CitAÇÃO DA PÁG. 7:

Há várias igualdades elementares que se pode escrever sobre funções $\delta$. Estas igualdades são essencialmente regras de manipulação para o trabalho algébrico envolvendo 
funções $\delta$. O sentido de cada uma destas igualdades é que seus dois membros fornecem resultados equivalentes como fatores em um integrando.

CITAÇÃo DA PÁG. 10:

A fim de obter uma imagem de $\delta(x)$, tome uma função da variável real $x$ que se anule fora de um pequeno domínio, digamos de comprimento $\varepsilon$, em torno da origem $x=0$, e que seja tão grande dentro deste domínio a ponto de sua integral sobre ele ser igual à unidade. A forma exata da função dentro deste domínio não importa, desde que não haja variações brutais desnecessárias (por exemplo, desde que a função seja sempre de ordem $\left.\varepsilon^{-1}\right)$. Então no limite $\varepsilon \rightarrow 0$ esta função tenderá a $\delta(x)$.

CitAÇÃO DA PÁG. 11:

... Poder-se-ia então eliminar completamente as funções impróprias. Portanto, o uso de funções impróprias não implica qualquer falta de rigor na teoria, sendo meramente uma notação conveniente, que nos permite expressar de forma concisa certas relações que poderíamos, se necessário, reescrever de uma forma que não envolvesse funções impróprias, mas que seria então desajeitada e tenderia a obscurecer a discussão.

CitaÇÕes DA PÁG. 15:

É demonstrado neste livro que as idéias de Leibniz podem ser completamente legitimadas, e que elas levam a uma nova e frutífera abordagem da Análise clássica e de muitos outros ramos da matemática. A chave para o nosso método é dada pela análise detalhada da relação entre linguagens matemáticas e estruturas matemáticas, a qual se encontra na base da teoria dos modelos contemporânea.

Pode-se esperar que alguns ramos da Física Teórica moderna, em particular aqueles afligidos por problemas de divergência, possam ser tratados com proveito pela Análise Não-standard. O fato do presente livro conter apenas aplicações à Matemática Aplicada clássica é muito provavelmente um testemunho das limitações do autor, e não do método. 


\section{Bibliografia}

[1] Courant, R., \& D. Hilbert: Methods of Mathematical Physics, vol. II, Interscience, New York, 1962.

[2] Dirac, P. A. M.: The Principles of Quantum Mechanics, third edition, Oxford at the Clarendon Press, 1947.

[3] Feynman, R. P.: Space-Time Approach to Non-Relativistic Quantum Mechanics, Rev. Mod. Physics., 20, 367-387, 1948.

[4] Keller, J. B. \& D. W. McLaughlin: The Feynman Integral, Am. Math. Monthly, 82, 451-465, 1975.

[5] Kline, M.: Mathematics Thought from Ancient to Modern Times, Oxford University Press, New York, 1972.

[6] Kline, M.: Mathematics, The Loss of Certainty, Oxford University Press, New York, 1980.

[7] Kuhn, T. S.: A Estrutura das Revoluções Científicas, Perspectiva, São Paulo, 1989.

[8] Laugwitz, D.: Anwendungen unendlich kleiner Zahlen, I, J. Reine Angew. Math., 207, 53-60, 1961.

[9] Laugwitz, D.: Anwendungen unendlich kleiner Zahlen, II, J. Reine Angew. Math., 208, 22-34, 1961.

[10] Lützen, J.: The Prehistory of the Theory of Distributions, Springer-Verlag, New York, 1982. 
[11] Robinson, A.: Non-Standard Analysis, North-Holland, Amsterdam, 1966.

[12] Schmieden, C., \& D. Laugwitz: Eine Erweiterung der Infinitesimalrechnung, Math. Zeitschr., 69, pp. 1-39, 1958.

[13] Schulman, L. S.: Techniques and Applications of Path Integration, John Wiley \& Sons, New York, 1981.

[14] Zygmunt, J.: On the Sources of the Notion of the Reduced Product, Rep. Math. Logic, 1, 53-67, 1973. 